University of Louisville

ThinkIR: The University of Louisville's Institutional Repository

$5-2020$

\title{
The role of Slc7a11 in controlling extracellular and intracellular redox environments of lung fibroblasts - potential targets for intervention in aging and idiopathic pulmonary fibrosis.
}

Yuxuan Zheng

University of Louisville

Follow this and additional works at: https://ir.library.louisville.edu/etd

Part of the Biochemistry Commons, Pharmacology, Toxicology and Environmental Health Commons, and the Pulmonology Commons

\section{Recommended Citation}

Zheng, Yuxuan, "The role of Slc7a11 in controlling extracellular and intracellular redox environments of lung fibroblasts - potential targets for intervention in aging and idiopathic pulmonary fibrosis." (2020). Electronic Theses and Dissertations. Paper 3428.

https://doi.org/10.18297/etd/3428

This Doctoral Dissertation is brought to you for free and open access by ThinkIR: The University of Louisville's Institutional Repository. It has been accepted for inclusion in Electronic Theses and Dissertations by an authorized administrator of ThinkIR: The University of Louisville's Institutional Repository. This title appears here courtesy of the author, who has retained all other copyrights. For more information, please contact thinkir@louisville.edu. 


\title{
THE ROLE OF SLC7A11 IN CONTROLLING EXTRACELLULAR AND INTRACELLULAR REDOX ENVIRONMENTS OF LUNG FIBROBLASTS - POTENTIAL TARGETS FOR INTERVENTION IN AGING AND IDIOPATHIC PULMONARY FIBROSIS
}

By

\author{
Yuxuan Zheng \\ Bachelor of Clinical Medicine, Jilin University, 2014 \\ Master of Internal Medicine, Jilin University, 2016
}

\begin{abstract}
A Dissertation
Submitted to the Faculty of the

School of Medicine of the University of Louisville for the Degree of

Doctor of Philosophy

in Pharmacology and Toxicology

Department of Pharmacology and Toxicology

University of Louisville

Louisville, KY
\end{abstract}

May 2020 
Copyright 2020 by Yuxuan Zheng

All rights reserved 

THE ROLE OF SLC7A11 IN CONTROLLING EXTRACELLULAR AND INTRACELLULAR REDOX ENVIRONMENTS OF LUNG FIBROBLASTS POTENTIAL TARGETS FOR INTERVENTION IN AGING AND IDIOPATHIC PULMONARY FIBROSIS

\author{
By \\ Yuxuan Zheng \\ Bachelor of Clinical Medicine, Jilin University, 2014 \\ Master of Internal Medicine, Jilin University, 2016
}

Dissertation Approved on

April 9, 2020

By the following Dissertation Committee

Walter H. Watson, Ph.D.

Jesse Roman, M.D.

J. Christopher States, Ph.D.

Lu Cai, M.D., Ph.D.

Shao-yu Chen, Ph.D.

Gary W. Hoyle, Ph.D. 


\section{DEDICATION}

This dissertation is dedicated to my parents, Xiaoling Fan and Gang Zheng. You believe in me and support every decision I made. Without your emotional and financial support, I could not be able to pursue this higher degree. I love you. Additionally, this dissertation is dedicated to my grandparents, Zhiyun Li, Fuchang Zheng, Ling Yang and Yong Fan. You helped me explore the world when I was a little boy. Your love makes me an honest and kind person. I remember my helplessness when some of you got sick and I could not do anything. I feel it's my responsibility to learn the science of life and medicine so that I can help you, not only you but also other people with same conditions and their families both physically and emotionally in the future. This is my life-long goal and I won't forget this. 


\section{ACKNOWLEDGEMENTS}

I would like to first thank my mentor Dr. Walter H. Watson. He introduced me to science and taught me every aspect of research. I learned another culture and way of thinking from him. He also develops my ability to continue working in the research field. I'm grateful for his guidance. I also want to thank Dr. Jesse Roman and Dr. J. Christopher States for providing great suggestions for my project and career. It is a great opportunity to learn how questions are approached from different perspectives of a physician and a scientist. Thanks to my other committee members, Dr. Lu Cai, Dr. Shao-yu Chen and Dr. Gary W. Hoyle for guiding me through this project and giving me good advices. Thanks to Dr. Jian Cai, Dr. Michael L. Merchant, Dr. Igor N. Zelko, Dr. Victor van Berkel, Dr. David R. Nunley, Dr. Andrew J. Halayko, Dr. Adam E. Gaweda, Tom Burke, Jeffery D. Ritzenthaler, Edilson Torres-Gonzalez, Javier Otero for providing help for the experiments in this dissertation.

Studying aboard in a new culture is hard. Thanks to Dr. Hongxue Shi for helping me set up my life in Louisville. He is the first contact I have in Louisville and his experience and advices as a senior student benefit me a lot. Thanks to my 10-year-old friends, Dr. Jian Shi, who encourages me to face difficulties with a positive attitude and go for my dreams, and Dr. Pu Shao, who shows me to 
stay cool and take advantage of the surrounding resources. Thanks to Dr. Zhisen Tian for being my alcohol buddy and helping me whenever I have trouble in Louisville. Thanks to my other friends, Dr. Chenxi Wu, Dr. Yongjun Zhang, Dr. Mengfei Li, Yihong Li, Dr. Huadong Fan, Dr. Mayukh Banerjee, Dr. Ana P. Ferragut Cardosoa, Ageliki Lykoudi, Dr. Lexiao Jin, Dr. Liya Chen, Fengyuan Li, Dr. J. Caleb Greenwell and Dr. Ryan C. McAllister. They make my Ph.D. journey enjoyable and amazing. Last but not least, thanks to my idol, Chaoyue Yang. Her attitude towards life is inspiring and she tells her fans that we will never give up. 


\begin{abstract}
THE ROLE OF SLC7A11 IN CONTROLLING EXTRACELLULAR AND INTRACELLULAR REDOX ENVIRONMENTS OF LUNG FIBROBLASTS POTENTIAL TARGETS FOR INTERVENTION IN AGING AND IDIOPATHIC

\section{PULMONARY FIBROSIS}

Yuxuan Zheng

April 9, 2020

Idiopathic pulmonary fibrosis (IPF) is a fatal lung disease characterized by extracellular matrix deposition by fibroblasts. Aging and oxidative stress increase the susceptibility to IPF. Redox couples, cysteine/cystine (Cys/CySS) and glutathione/glutathione disulfide (GSH/GSSG), and their redox potentials $\left(E_{h}\right)$ quantify oxidative stress. Fibroblasts from old mice maintain more oxidized extracellular $E_{h}($ Cys/CySS) than young mice. Microarray shows downregulation of Slc7a11 potentially mediates this age-related oxidation. Slc7a11 is the key component of system $X_{c}^{-}$, an antiporter that imports CySS and exports glutamate. The first aim of this dissertation is to investigate the mechanistic link between Slc7a11 expression and extracellular $E_{h}(C y s / C y S S)$. The second aim is to evaluate the effects of aging on the redox states of intracellular proteins and whether Slc7a11 contributes to the age-dependent effects. The last aim is to compare SLC7A11 expression, extracellular 
$E_{h}\left(\right.$ Cys/CySS) and intracellular $E_{h}(G S H / G S S G)$ between human lung fibroblasts from IPF and non-IPF donors and to explore their association with pro-fibrotic gene expression.

Slc7a11 expression was manipulated by pharmacological and genetic methods. Reduced and oxidized forms of Cys residues were labelled by lodoacetyl Tandem Mass Tags. The ratio of oxidized/reduced forms (i.e., redox state) of a Cys residue was determined by multiplexed tandem mass spectrometry.

$E_{h}\left(\right.$ Cys/CySS) and $E_{h}(G S H / G S S G)$ were more oxidized in conditioned media of old fibroblasts. Up-regulation of Slc7a11 reduced extracellular $\mathrm{Eh}$ (Cys/CySS) for old fibroblasts. Inhibition of GSH synthesis had no effect on the ability of cells to restore their extracellular $\mathrm{E}_{h}$ (Cys/CySS). Redox states of 151 proteins changed with aging. Slc7a11 over-expression restored redox states of 104 proteins. Ingenuity Pathway Analysis showed these 104 proteins were involved in pathways of protein translation initiation, ubiquitinproteasome-mediated degradation and integrin-cytoskeleton-associated signaling. Slc7a11 expression was lower in IPF fibroblasts. Extracellular $E_{h}($ Cys/CySS) was more oxidized and expression of pro-fibrotic genes was higher in IPF fibroblasts.

In conclusion, Slc7a11 is the key regulator of extracellular $E_{h}$ (Cys/CySS). Its effects are independent of GSH synthesis. Aging results in changes of redox states of proteins involved in protein turnover and cytoskeleton dynamics. Up- 
regulating Slc7a11 restores changes of protein redox states due to aging.

Decreased SLC7A11 might represent a susceptibility factor for developing tissue disrepair and fibrosis in IPF. 


\section{TABLE OF CONTENTS}

PAGE

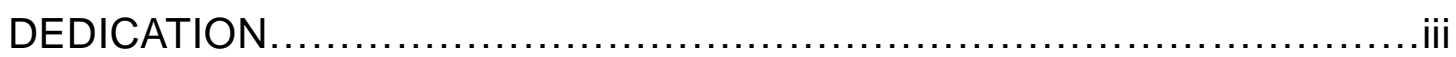

ACKNOWLEDGEMENTS ........................................................

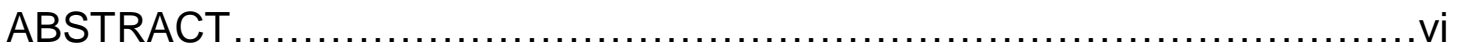

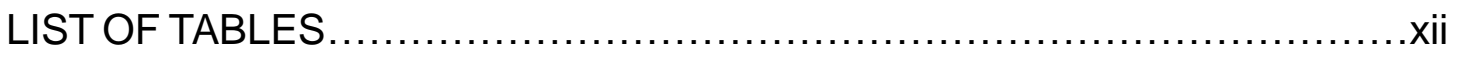

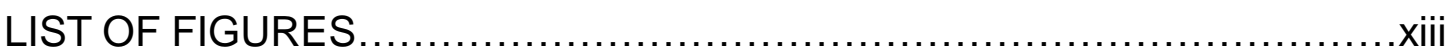

CHAPTER I. INTRODUCTION

1.1 Overview of idiopathic pulmonary fibrosis (IPF) ............................

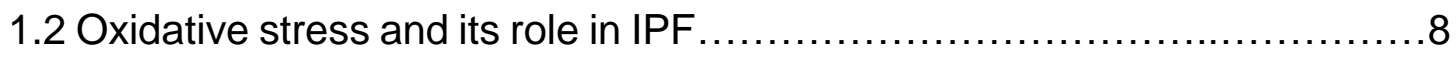

1.3 Redox couples and their roles in lung fibrosis..............................15

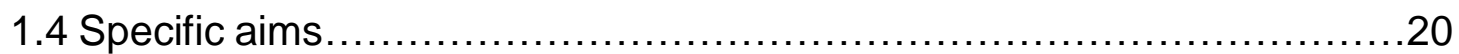

CHAPTER II. AGE-DEPENDENT OXIDATION OF EXTRACELLULAR CYSTEINE/CYSTINE REDOX STATE (EH(CYS/CYSS)) IN MOUSE LUNG FIBROBLASTS IS MEDIATED BY A DECLINE IN SLC7A11 EXPRESSION

2.1 Introduction

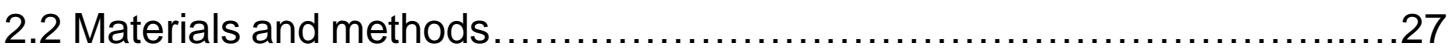

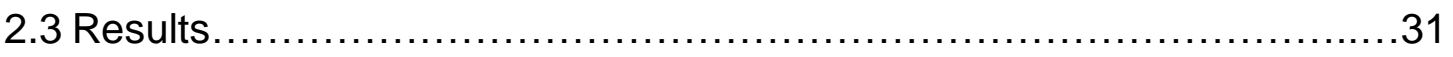

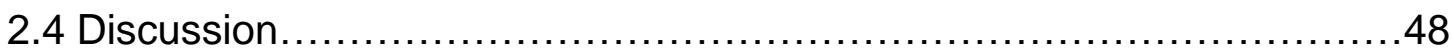


CHAPTER III. REDOX STATES OF PROTEIN CYSTEINES IN PATHWAYS OF PROTEIN TURNOVER AND CYTOSKELETON DYNAMICS ARE CHANGED WITH AGING AND REVERSED BY SLC7A11 RESTORATION IN MOUSE LUNG FIBROBLASTS

3.1 Introduction .53

3.2 Materials and methods

3.3 Results.

3.4 Discussion.

CHAPTER IV. DECREASED SLC7A11 IS ASSOCIATED WITH OXIDATION OF EXTRACELLULAR CYSTEINE/CYSTINE REDOX STATE (EH(CYS/CYSS)) IN HUMAN LUNG FIBROBLASTS: A POTENTIAL SUSCEPTIBILITY STATE FOR IDIOPATHIC PULMONARY FIBROSIS

4.1 Introduction .88

4.2 Materials and methods .92

4.3 Results. .95

4.4 Discussion. .104

CHAPTER V. SUMMARY AND DISCUSSION

5.1 Slc7a11 and its role in regulating redox environments for lung fibroblasts.

5.2 Strengths and weaknesses 118

5.3 Future directions .120 REFERENCES 


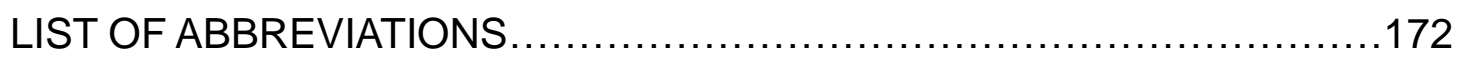

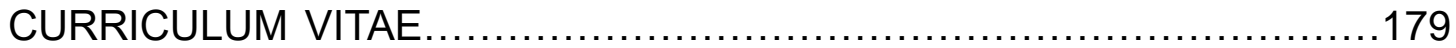




\section{LIST OF TABLES}

PAGE

Table 3.1 Ingenuity Pathway Analysis (IPA) indicated proteins whose redox states were restored by Slc7a11 overexpression were enriched in pathways of protein turnover and cytoskeleton signaling .73

Table 3.2 GO-based biological process analysis of proteins whose redox states were rescued by Slc7a11 overexpression in old fibroblasts. .75

Table 3.3 GO-based molecular function analysis of proteins whose redox states were rescued by Slc7a11 overexpression in old fibroblasts .76

Table 3.4 GO-based cellular component analysis of proteins whose redox states were rescued by Slc7a11 overexpression in old fibroblasts. .77 Table 3.5 KEGG pathway analysis of proteins whose redox states were rescued by Slc7a11 overexpression in old fibroblasts. .78 Table 4.1 Age and sex of IPF patients and non-IPF donors. .93 


\section{LIST OF FIGURES}

PAGE

Figure 2.1 Sulforaphane treatment of old fibroblasts restored Slc7a11 expression and extracellular $\mathrm{E}_{\mathrm{h}}(\mathrm{Cys} / \mathrm{CySS})$ to the levels seen in young fibroblasts 33

Figure 2.2 Glutathione was less abundant and more oxidized in the conditioned media of old fibroblasts, and sulforaphane corrected these deficiencies. .35 Figure 2.3 Reduction of extracellular Eh(Cys/CySS) in response to sulforaphane is mediated by Slc7a11 .38

Figure 2.4 Slc7a11 inhibition by sulfasalazine in young mice lung fibroblasts resulted in oxidation of extracellular $\mathrm{E}_{\mathrm{h}}$ (Cys/CySS)..... .40

Figure 2.5 Slc7a11 transport activity was increased by sulforaphane and inhibited by sulfasalazine.

Figure 2.6 Effect of genetic manipulation of Slc7a11 on extracellular $E_{h}($ Cys/CySS)

Figure 2.7 Overexpression of Slc7a11 increased, and knock down of Slc7a11 decreased, extracellular Cys concentrations.

Figure 2.8 Glutathione depletion with BSO did not affect extracellular Cys concentration or $\mathrm{Eh}_{\mathrm{h}}$ Cys/CySS).

Figure 3.1 Overexpression of Slc7a11 restored age-dependent oxidation of 
extracellular $\mathrm{E}_{\mathrm{h}}$ (Cys/CySS) in old fibroblasts.

Figure 3.2 Schematic flow chart of labeling redox-sensitive cysteines in peptides from young, old and old Slc7a11-overexpressing primary mouse lung fibroblasts using iodoTMT reagents.

Figure 3.3 Representative mass spectrum for determining Cys redox state....66 Figure 3.4 Volcano plot showing peptides with age-dependent Slc7a11reversible Cys redox states 69

Figure 3.5 Protein-protein interaction (PPI) network showed proteins with agedependent Slc7a11-responsive cysteines were clustered in IPA-predicted pathways.

.80

Figure 4.1 Extracellular Eh(Cys/CySS) became more oxidized for lung fibroblasts from IPF patients due to lower extracellular Cys concentration......97 Figure 4.2 SLC7A11 expression was lower for IPF fibroblasts. 100

Figure 4.3 Expressions of pro-fibrotic genes were higher for IPF fibroblasts .101

Figure 4.4 Intracellular $E_{h}(G S H / G S S G)$ was not more oxidized for IPF fibroblasts 103

Figure 5.1 Connection between Cys/CySS and GSH/GSSG redox couples. .114 


\section{CHAPTER I}

\section{INTRODUCTION}

1.1 Overview of idiopathic pulmonary fibrosis (IPF)

Idiopathic pulmonary fibrosis (IPF) is characterized by scarring of the lung without known causes. Normally, the connective tissues between alveoli are very thin so that oxygen can diffuse effectively. However, IPF is characterized by irreversible destruction of alveoli and remodeling of lung with excessive synthesis and deposition of extracellular matrix (ECM) in the interstitial spaces (1). As the interstitium becomes thicken, the function of the lung for gas exchange gradually declines. According to the American Thoracic Society (ATS) guideline, IPF is defined as "a specific form of chronic, progressive fibrosing interstitial pneumonia of unknown cause, occurring primarily in older adults, limited to the lungs, and associated with histopathologic and/or radiologic pattern of usual interstitial pneumonia (UIP)" (2).

Generally, the pathological pattern of IPF is UIP. Although not pathognomonic, pathological UIP increases the confidence of IPF diagnosis if radiological UIP pattern is suspected but not well established after patient history is thoroughly reviewed and potential causes are excluded (3). Fibrosis 
in IPF is primarily located at the periphery of the lower regions of both lungs. Two distinctive characteristics of UIP are honeycombing and fibroblast foci (4). Honeycombing refers to the enlarged air cysts lined with bronchiolar-type epithelium and filled with mucin and inflammatory cells, while fibroblast foci are aggregates of fibroblasts and myofibroblasts that are actively producing matrix proteins (5).

The increasing prevalence and fatality of IPF require people's awareness. There are over 180,000 IPF patients in the United States (6). According to an epidemiological study among Medicare beneficiaries over 65 years of age, the prevalence of IPF increased dramatically from 202.2 cases per 100,000 personyears in 2001 to 494.5 cases per 100,000 person-years in 2011 (7). Furthermore, IPF is a disease of aging. It predominantly develops in people over 55 years old and its incidence increases with aging $(8,9)$. The median age at diagnosis is 66 years old (10). Sex and ethnicity also affect susceptibility to IPF. The incidence in males is higher than that of females (9). Caucasians and Hispanics are more susceptible than African-Americans and Asians (7). Mortality also increases with aging and is higher in Caucasians $(7,11)$. The survival of IPF is worse than most cancers. The median survival time is 2 to 3 years $(10,12)$. Its five-year survival is only higher than pancreatic cancer and lung cancer (13).

The diagnosis of IPF is complex and dependent on a multidisciplinary approach. Patients usually present with shortness of breath, dry cough and 
fatigue, which are chronic but non-specific. Typically, bibasilar inspiratory crackles are present in physical exam. Basic information and history of the patient, including age, sex, smoking status, family history and environmental exposures need to be carefully evaluated to exclude potential causes. Highresolution computed tomography (HRCT) is usually required. Based on whether the pattern of HRCT is UIP, how confident the UIP pattern is established and whether surgical lung biopsy is practical, multidisciplinary discussion is suggested to determine diagnosis and further management of the patient $(3,14)$.

There is no cure for IPF, but several treatments are available to increase patients' wellbeing or slow down IPF progression. Pulmonary rehabilitation, including exercise, emotional support and education, aims to improve overall physical health and quality of life $(15,16)$. Supplemental oxygen becomes necessary during the IPF course at some point (17). Pirfenidone and nintedanib are the two US FDA approved anti-fibrotic drugs. Their major effect is to slow down deterioration of patients' lung function $(18,19)$. Side effects include nausea and anorexia for pirfenidone and diarrhea for nintedanib. Both drugs affect cytochrome P450. Therefore, usage of other drugs and liver function should be monitored. Though lung transplantation is another option, the posttransplant survival remains low (20).

Pathogenesis of IPF is multifaceted. Current paradigm focuses on aberrant wound healing and lung remodeling affected by various factors, including 
genetics, aging, environmental exposures, oxidative stress, interplay between epithelial cells, inflammatory cells and fibroblasts via cytokines, and interaction between cells and the environments (matrix or redox) they reside in.

Gene polymorphism and mutation determines susceptibility to IPF. A single nucleotide polymorphism guanine to thymine in MUC5B promoter has been confirmed to increase susceptibility (21). Patients with familial pulmonary fibrosis present symptoms earlier than sporadic IPF (22). Genes encoding surfactant, telomerase and innate immune-related toll interacting protein are also associated with IPF (23-26).

Aging is the most important host factor for increasing IPF susceptibility. Aging is characterized by impaired physiological function and degenerated biological structure (27). Age-related deficiencies, such as oxidative stress, genetic instability and epigenetic change are all identified in IPF epithelial cells or fibroblasts, suggesting the processes of aging overlap with and promote development of IPF (28). Lung fibroblasts isolated from IPF patients exhibit cellular senescence compared to those from their age-matched controls (29). Bleomycin induces more severe lung fibrosis in old mice than young mice via activating pro-fibrotic TGF- $\beta 1 /$ Smad signaling pathway (30).

Recurrent environmental exposures induce repetitive damage that contribute to IPF development. Cigarette smoke, farming, livestock, dust of wood, metal, stone, sand and silica all increase IPF risk (31). Among them, cigarette smoke has the highest public health relevance because of the large 
smoking population (32). Other proposed risk factors for recurrent microinjuries include viral infection, gastroesophageal reflux, change of lung microbiome, diabetes mellitus and obstructive sleep apnea (33). Those risk factors are mostly identified by retrospective case-control studies. Therefore, causal relationships cannot be established.

Redox homeostasis is disrupted in IPF and oxidative stress has been implicated in IPF. The lungs are exposed to oxygen directly and sensitive to oxidative stress. Multiple biomarkers of oxidative stress, including protein carbonyls, 8-isoprostane and hydroperoxide, are increased (34). Oxidative stress also induces DNA damage, ECM fragmentation and pro-fibrotic cytokine activation, all of which promote aberrant wound healing and may increase the susceptibility to develop IPF (34).

Injured epithelial cells are considered initiators of IPF. In the alveoli, most epithelial cells are type I alveolar epithelial cells (AECs). They maintain the shape of alveoli and allow for gas exchange. Damage of type I AECs signals the progenitor type II AECs to regenerate type I AECs to restore normal lung structure and function. However, during IPF development, repetitive microinjuries result in exhaustion of type II AECs (35). Epithelial reprogramming occurs and is accompanied by change of epithelial morphology (36). Reepithelialization is dysregulated, resulting in pathological honeycombing (37). Embryonic development pathways, such as Wnt and Sonic hedgehog pathways, are also reactivated $(38,39)$. Epithelial cells secret chemokines to 
recruit fibroblasts, growth factors and pro-fibrotic cytokines to induce proliferation, migration and activation of fibroblasts $(37,40)$. They also secret procoagulants to increase the risk of thromboembolism (41). Unfolded protein response and endoplasmic reticulum stress induce epithelial apoptosis and epithelial-mesenchymal transition, which play critical roles in IPF pathogenesis $(42,43)$.

The role of inflammatory cells in IPF is controversial. There is only mild inflammation in IPF, and anti-inflammatory therapies are not effective (44). Whether inflammatory cells are pro- or anti-fibrotic depends on context and mediators they secret (45).

Fibroblasts are the effector cells. Fibroblasts isolated from lungs of IPF patients have slower growth rate, flattened shape and decreased telomere length, indicating replicative senescence $(29,46)$. They are also activated to differentiate into more contractile, invasive and apoptosis-resistant myofibroblasts (47). Myofibroblasts not only originate from local resident fibroblasts, but also from other resources including circulating bone marrow derived fibrocytes, pericytes and epithelial- or endothelial-mesenchymal transition (48). Fibroblasts and myofibroblasts form fibrotic foci, where they produce exuberant and aberrant ECM proteins and determine the fibrotic phenotype of IPF $(49,50)$.

The behavior of fibroblasts is dependent on the interaction between fibroblasts and their surrounding environment. Serum deprivation-induced 
fibroblast apoptosis is blocked by TGF- $\beta$ (51). Furthermore, adherent fibroblasts express $\alpha$-smooth muscle actin ( $\alpha$-SMA) in response to TGF, while suspended fibroblasts lose such response (52).

ECM is one important factor that determines fibroblast phenotype. The function of matrix is to maintain structural framework and elasticity of the lung. Fibroblasts, which produce ECM, are prone to cluster in areas with higher matrix stiffness, resulting in aggravation of IPF via a positive feedback loop (53). Fibroblasts display elongated and dendritic shape, increased proliferation and migration, decreased apoptosis, increased collagen expression and decreased expression of proteolysis-associated genes on substrate with higher stiffness (53).

Comparable to the matrix environment, the redox environment is another important factor that changes fibroblast behavior. This will be discussed in detail in the following sections.

No ideal animal models are available for simulating human IPF. Current animal models are based on injury-induced lung fibrosis without idiopathic or spontaneous nature. Different models have their own advantages and disadvantages (54). The most commonly used model is intratracheal bleomycin-induced lung fibrosis in mice (55). Bleomycin causes breaks of DNA strands and generation of free radicals specifically in the lung due to lack of its hydrolase. The major limitations of this model are that bleomycin induces considerable inflammation and the fibrosis is self-limited, both of which are not 
consistent with IPF (56). This model can be divided into 3 stages including proinflammatory phase, transitional phase and fibrotic phase (57). Other chemicals to induce lung fibrosis include silica, radiation, asbestos, paraquat and fluorescent isothiocyanate (58).

1.2 Oxidative stress and its role in IPF

Oxidative stress is classically defined as "an imbalance between prooxidants and anti-oxidants in favor of the former" (59). Although there are some discrepancies, a plethora of studies have shown oxidative stress is increased in IPF and contributes to its pathogenesis $(34,60)$.

Generally, pro-oxidants are increased. Compared to clinical samples from healthy donors, different kinds of reactive oxygen species (ROS) and oxidation products of lipids, proteins and DNA are increased in one or more IPF patientsderived samples, including bronchoalveolar lavage (BAL), epithelial lining fluid $(E L F)$, exhaled breath condensate (EBC), plasma, serum, urine, sputum, breath, specific type of lung cells and whole lung tissue. Superoxide anion $\left(\mathrm{O}_{2}^{-}\right)$ is increased in inflammatory cells from BAL (61). Hydrogen peroxide $\left(\mathrm{H}_{2} \mathrm{O}_{2}\right)$ is increased in BAL, EBC and serum (61-64). 8-isoprostane is increased in BAL, EBC, plasma and serum (65-68). Carbonyls are increased in BAL $(69,70)$. Nitrotyrosine is increased in EBC, epithelial cells and inflammatory cells (66, 71). Ethane is increased in breath (72). 8-hydroxy-deoxyguanosine (8-OHdG) is increased in epithelial cells (73). Some studies stratify subjects into smokers and non-smokers. Malondialdehyde (MDA) is increased in plasma of non- 
smokers with IPF compared to healthy non-smokers (74).

On the other hand, non-enzymatic and enzymatic anti-oxidants are mostly decreased in IPF samples.

Concentrations of reduced glutathione (GSH) and its oxidized form, glutathione disulfide (GSSG) are the most commonly measured non-enzymatic small molecular anti-oxidants. GSH is decreased in plasma (75). GSSG is increased in BAL and whole blood $(76,77)$. Total GSH (GSH + 2GSSG) is decreased in ELF, sputum and whole blood (77-80). The ratio of GSH to GSSG is decreased in whole blood (77). When smoking status is considered, GSH is decreased in BAL from non-smoking IPF patients than non-smoking health controls (74). It's obvious that the balance of GSH/GSSG redox couple is disrupted in IPF. GSH is a tripeptide synthesized from cysteine, glutamate and glycine. In IPF lung tissue, these three amino acids are increased while GSH synthesis enzymes, including glutamate-cysteine ligase $(\mathrm{GCL})$ and glutathione synthetase (GSS), are decreased (81). Trolox equivalent anti-oxidant capacity (TEAC), a measurement of total anti-oxidant capacity, decreased in both BAL and plasma from IPF patients in non-smoking subjects (74). Surprisingly, vitamins $A, C, E$ and uric acid, which are generally considered anti-oxidants, are increased in BAL from IPF patients (76).

Expression or activity of multiple anti-oxidant enzymes are changed in IPF, further disrupting redox homeostasis. Catalase (CAT) metabolizes $\mathrm{H}_{2} \mathrm{O}_{2}$ to water $\left(\mathrm{H}_{2} \mathrm{O}\right)$ and oxygen $\left(\mathrm{O}_{2}\right)$. Both CAT expression and activity are decreased 
in IPF lung tissue (82). Glutathione peroxidase (GPX) reduces $\mathrm{H}_{2} \mathrm{O}_{2}$ using $\mathrm{GSH}$. GPX3 is up-regulated in IPF lung tissue (83). Superoxide dismutase (SOD) transforms $\mathrm{O}_{2}{ }^{-}$to $\mathrm{H}_{2} \mathrm{O}_{2}$ and $\mathrm{O}_{2}$. SOD3 is decreased in fibrotic areas of the IPF lung tissue compared to non-fibrotic areas (84). However, SOD1 is increased in the serum of IPF patients (85). Glutaredoxin (GRX) uses GSH to reduce protein S-glutathionylation (deglutathionylation). GRX expression and activity are decreased in IPF lung tissue (86). Thioredoxin (TRX) reduces intra- or intermolecular disulfide formed from two protein cysteine residues. TRX reductase regenerates reduced TRX using electrons from reduced nicotinamide adenine dinucleotide phosphate (NADPH) (87). Immunochemistry staining shows neither TRX nor TRX reductase is expressed in normal alveolar epithelial cells (88). However, both TRX and TRX reductase are increased in metaplastic alveolar epithelial cells in IPF (88). Peroxiredoxin (PRX) metabolizes organic hydroperoxide $(\mathrm{ROOH})$ and $\mathrm{H}_{2} \mathrm{O}_{2}$ to $\mathrm{H}_{2} \mathrm{O}$ or alcohol $(\mathrm{ROH})$. Overall PRXII is decreased in IPF lung tissue (89). However, PRXII expression is increased in hyperplastic epithelium but decreased in fibroblasts foci (89). Sulfiredoxin (SRX) reduces cysteine sulfinic acid to sulfenic acid. SRX1 is increased in IPF lung tissue (90). Those cysteine-reducing enzyme systems are interconnected. TRX and GRX systems can both reduce cysteine disulfide or sulfenic acid back to thiols, regenerating functional PRX (91). SRX can also activate PRX via reducing cysteine sulfinic acid and use GSH to catalyze deglutathionylation, as with GRX $(92,93)$. Even they share similar functions and their substrates 
overlap, these reducing systems are not entirely replaceable with each other since their reducing mechanisms are different and their substrate specificity remains incompletely understood (94).

Although many anti-oxidants are decreased, the fact that some of these anti-oxidants are still increased and that expression of the anti-oxidant enzymes are cell type-specific and location-specific in IPF lungs suggest that redox disturbance in IPF is not simply due to a decrease in anti-oxidants, and that intricate redox-related regulatory mechanisms exist. Systems controlling intracellular and extracellular redox environments might be functionally connected with each other but regulated independently. And those systems could be differentially impaired during IPF pathogenesis.

Why oxidative stress is increased and how redox imbalance promotes lung disrepair and fibrosis are incompletely elucidated.

NADPH oxidases (NOXs) and mitochondria are the two major sources of ROS. Studies based on animal models of lung fibrosis have shown activity of different isoforms of NOXs are increased, resulting in increased production of ROS (95). Among them, NOX4 is the only isoform that has been confirmed to be upregulated in fibroblasts and smooth muscle cells from IPF lungs $(96,97)$. Dysfunctional mitochondria are another source of excessive ROS (98). $\mathrm{O}_{2}$ is partially reduced by electrons leaked from electron transport chain (99). Furthermore, there is an interplay between mitochondria and NOXs, which further aggregates ROS production (60). 
Generally, increased oxidative stress induces epithelial apoptosis, promotes differentiation of fibroblasts into myofibroblasts to secret more extracellular matrix proteins, recruit inflammatory cells and disrupt the balance between pro- and anti-fibrotic cytokines. Oxidative stress also results in chemical modifications of lung matrix proteins, changing not only the structure and function of specific proteins, but also the biomechanics that might affect the interaction between fibroblasts and matrices.

There is a vicious cycle between redox imbalance and TGF- $\beta$ signaling (100). TGF- $\beta$ is the major pro-fibrotic cytokine. It is present extracellularly in the inactive form consisting of dimeric TGF- $\beta$, latency-associated protein (LAP) and latent TGF- $\beta$ binding protein (LTBP). ROS can activate TGF- $\beta$ via modifying and dissociating LAP directly or indirectly via matrix metallopeptidase (MMP) mediated LAP cleavage $(101,102)$. ROS can also induce TGF- $\beta$ expression $(103,104)$. On the other hand, TGF- $\beta$ promotes redox imbalance. NOX4 is upregulated by TGF- $\beta$ in fibroblasts from control and IPF patients (105). Mitochondria-derived ROS induced by TGF- $\beta$ results in mitochondrial DNA damage and dysfunction in fibroblasts, promoting maladaptation and lung fibrosis (106). TGF- $\beta$ also decreases GSH synthesis via inhibiting both catalytic and modifier subunits of glutamate-cysteine ligase (GCLC and GCLM) in mouse lung (107). In terms of anti-oxidant enzymes, GRX1 is down-regulated by TGF- $\beta$ in A549 cells, an alveolar epithelial cell line derived from lung adenocarcinoma, and expressions of SOD2 and CAT are suppressed by TGF- 
$\beta$ in airway smooth muscle cells $(108,109)$.

Many pro-fibrotic effects on fibroblasts are dependent on ROS. Elimination of NOX4-produced ROS by N-acetylcysteine (NAC) or inhibition of NOX4 blocks the effects of TGF- $\beta$ to induce collagen expression and myofibroblast differentiation of fibroblasts (105). Serum-induced proliferation and invasion of IPF fibroblasts are blocked by NAC or NOX4 knockdown (110). Even without treatments, silencing NOX4 results in decreased basal expression of procollagen, fibronectin and $\alpha$-SMA in IPF fibroblasts (110). ROS over-production due to mitochondrial dysfunction contributes to IPF fibroblast senescence (111113). Anti-oxidants targeting mitochondria attenuate induction of $\alpha$-SMA and NOX4 expression by TGF- $\beta$ in IPF fibroblasts (114). Exogenous $\mathrm{H}_{2} \mathrm{O}_{2}$ increases a-SMA expression in a dose- and time-dependent manner in control lung fibroblasts while NAC reduces expressions of $\alpha$-SMA and type-I collagen in early passages of IPF fibroblasts (115).

ROS also mediates pro-fibrotic effects on other cell types and modifies matrix proteins. Anti-oxidants abrogates the effects of exogenous $\mathrm{H}_{2} \mathrm{O}_{2}$ to induce epithelial-to-mesenchymal transition (EMT) for A549 cells (116). SOD1mediated production of $\mathrm{H}_{2} \mathrm{O}_{2}$ polarizes macrophages to the $\mathrm{M} 2$ phenotype, promoting lung remodeling and fibrosis (117). $\mathrm{H}_{2} \mathrm{O}_{2}$ secreted by IPF fibroblasts induces small airway epithelial cell death (118). Exogenous $\mathrm{H}_{2} \mathrm{O}_{2}$ inhibits wound healing in a concentration-dependent manner via inducing apoptosis and suppressing migration of alveolar epithelial cells (119). Bleomycin-generated 
ROS induces endothelial stress and differentiation of pericytes into myofibroblasts (120). ROS contributes to matrix dysregulation in IPF (121). Hyaluronic acid, which is increased in BAL from IPF patients, is prone to $\mathrm{H}_{2} \mathrm{O}_{2}$ induced degradation $(122,123)$. Syndecan-1 is increased in both BAL and lung tissue from IPF patients (124). Its shedding and function as neutrophil chemoattractant are inhibited by SOD1 (124).

Previous clinical trials targeting oxidative stress were unsuccessful. Compared to placebo, NAC monotherapy did not benefit IPF patients in terms of slowing down lung function deterioration or decreasing mortality (125). Surprisingly, NAC triple-therapy with prednisone and azathioprine increased the rate of death and hospitalization, and the study was terminated after interim analysis (44). The failure of those studies indicates providing exogenous antioxidants to decrease overall oxidative stress might not be the way to treat IPF since many oxidants have physiological roles and giving anti-oxidants indiscriminately may disrupt those normal biological functions. However, NAC monotherapy was related to a significant decrease of risk of death and disease progression in patients with a TT genotype of the TOLLIP gene that plays important roles in lung host defense (26). The genotype-stratified effect further reveals the complexity of the redox regulation in IPF. Moreover, considering IPF pathogenesis is multifactorial, and pro-fibrotic pathways overlap and compensate for each other, interventions with pleiotropic effects might have a better chance of being effective than highly specific drugs that target a single 
molecule or pathway (126). The ineffectiveness of overall anti-oxidants and highly selective drugs in general IPF patients facilitates us to think whether there will be other methods to intervene redox imbalance in a neither too general nor too specific manner. My proposed intervention against oxidative stress is manipulating redox couples and their redox potentials to restore redox homeostasis.

1.3 Redox couples and their roles in lung fibrosis

Redox couples provide a new aspect of studying oxidative stress. Traditional definition of oxidative stress emphasizes the overall imbalance between pro-oxidants and anti-oxidants. However, the sensitivity to oxidants and the response to anti-oxidants of different systems are not the same (127). Furthermore, traditional definition neglects the signaling role of redox modification and normal biological functions of specific redox molecules. Modern concept of oxidative stress adds the results of the redox imbalance, which are disruption of redox signaling and damage to biological molecules (128). Compared to ROS, redox couples have the advantage of being easier to measure and constituting a hierarchical regulatory network themselves (127). They can buffer oxidative stress and transduce signals via regulating redox environment or by affecting protein functions through oxidation and reduction of cysteine thiols $(129,130)$.

Based on concentrations, cysteine/cystine (Cys/CySS) is the major extracellular redox couple while GSH/GSSG is the major intracellular redox 
couple (131). There are several other intracellular redox couples as well, such as reduced/oxidized Trx and NADPH/NADP ${ }^{+}$(132). Redox potential (Eh) is used to quantitatively describe the reducing ability of a redox couple to buffer oxidative stress and is calculated from the Nernst equation. Those redox couples are not in equilibrium with each other, which is another evidence of inadequacy of traditional balance definition of oxidative stress (131). In fact, even for a particular redox couple, its redox potential differs significantly in subcellular compartments (133). Normal human plasma GSH/GSSG redox potential $\left(E_{h}(G S H / G S S G)\right)$ and plasma Cys/CySS redox couple (Eh(Cys/CySS)) are around $-140 \mathrm{mV}$ and $-80 \mathrm{mV}$, respectively (134). Cytoplasmic $\mathrm{Eh}(\mathrm{GSH} / \mathrm{GSSG})$ varies between $-220 \mathrm{mV}$ and $-260 \mathrm{mV}$ while cytoplasmic $\mathrm{E}_{\mathrm{h}}$ (Cys/CySS) is close to $-160 \mathrm{mV}$ (133). Mitochondrial $\mathrm{E}_{\mathrm{h}}(\mathrm{GSH} / \mathrm{GSSG}$ ) and nuclear $E_{h}(G S H / G S S G)$ are more reducing than cytoplasmic $E_{h}(G S H / G S S G)$ (135).

Though components of each redox couple are in dynamic status to maintain relatively stable redox potential, many factors can affect the set-point value of redox potential. Human plasma $E_{h}(G S H / G S S G)$ and $E_{h}\left(C_{y s} / C y S S\right)$ become progressively oxidized with aging $(136,137)$. They are also more oxidized in smokers compared to non-smokers (138). Plasma $E_{h}(G S H / G S S G)$ becomes oxidized over time with chemotherapy (139). Other risk factors related with oxidation of plasma $E_{h}(G S H / G S S G)$ or $E_{h}($ Cys/CySS) include high serum cholesterol level, low sulfur amino acid diet and alcohol consumption (140-142). 
Those data also support the concept that redox potentials respond to endogenous and exogenous disturbance and might be indicators reflecting combined effects of regulation of redox environment.

Change of redox potential is associated with change of cellular status and biological process, which might promote development of multiple diseases. Cytosolic $\mathrm{E}_{h}(\mathrm{GSH} / \mathrm{GSSG})$ becomes progressively oxidized during the life cycle when cells switch from proliferation $(-240 \mathrm{mV})$ to differentiation $(-200 \mathrm{mV})$ to apoptosis (-170 mV) (143). Oxidation of extracellular $E_{h}$ (Cys/CySS) from -150 $\mathrm{mV}$ to $0 \mathrm{mV}$ stimulates monocyte adhesion, inhibits colorectal adenocarcinoma epithelial proliferation and increases oxidant-induced retinal pigment epithelial apoptosis (144-146). Oxidized extracellular Eh(Cys/CySS) stimulates proinflammatory signaling in endothelial cells, while oxidized plasma $\mathrm{En}_{\mathrm{h}}(\mathrm{GSH} / \mathrm{GSSG})$ directly correlates with thickness of carotid intima-media thickness, both of which suggest increased chance of developing atherosclerosis $(147,148)$. Plasma $E_{h}(G S H / G S S G)$ is more oxidized in diabetic patients (149). For chronic obstructive pulmonary disease (COPD) patients, plasma $E_{h}($ Cys/CySS) is more oxidized (150). Furthermore, loss of lung function correlates with the extent of plasma $E_{h}$ (Cys/CySS) oxidation (150). Severity of human immunodeficiency virus (HIV) infection, which is represented by CD4-positve T cell count, also correlates with plasma $E_{h}$ (Cys/CySS) (150). Reduced extracellular $E_{h}(\mathrm{Cys} / \mathrm{CySS})$ decreases mouse neuronal death via increasing GSH and decreasing ROS (151). Reduced extracellular 
$\mathrm{E}_{\mathrm{h}}$ (Cys/CySS) also reverses the loss of free reduced nicotinamide adenine dinucleotide (NADH) in mouse old and Alzheimer's neurons, protecting against age-related Alzheimer's disease (152). Oxidized extracellular $E_{h}($ Cys/CySS) induces ROS generation, promotes extracellular signal-regulated kinase (ERK) phosphorylation and NF-kB expression via activating metabotropic glutamate receptor 5 (mGlu5), and stimulates inducible nitric oxide synthase (iNOS) expression in rat glioma C6 cells (153). The oxidized media conditioned by C6 glial cells decrease tyrosine hydroxylase expression in mouse dopaminergic neuronal MN9D cells, which is neurotoxic and promotes Parkinson's disease (153). However, reduced extracellular $E_{h}($ Cys/CySS) promotes migration and invasion of PC3 prostate carcinoma cells via increasing MMP9 activity and NOX1-mediated $\mathrm{H}_{2} \mathrm{O}_{2}$ production (154). Reduced $\mathrm{E}_{h}$ (Cys/CySS) also promotes proliferation of colon carcinoma Caco-2 cells via metalloproteinase-mediated epidermal growth factor receptor (EGFR) activation and downstream p44/p42 mitogen-activated protein kinase (MAPK) phosphorylation (155).

Overall, the effects of change of redox potential might be versatile and dependent on cells and tissues. Oxidizing redox potential might generally initiate diseases. Reducing redox potential might generally increase cellular defense against harmful stimulants, thus increasing cancer cell survival and promoting disease progression. However, whether oxidation or reduction is beneficial for patients is determined on a case-by-case basis and might be dependent on the disease course or stage. The underlying mechanisms are not 
well studied and might involve a combination of disturbing intracellular redox environments, influencing post-translational modifications and functions of proteins and affecting multiple signal transduction pathways.

Particularly, changes of redox potential contribute to lung fibrosis. Oxidized extracellular $E_{h}(\mathrm{Cys} / \mathrm{CySS})$ stimulates proliferation of primary mouse lung fibroblasts and fibronectin expression via activating protein kinase $\mathrm{C}$, upregulating TGF- $\beta 1$ and CAMP response element binding protein (CREB) (156). NF-kB and Smad3 levels are also increased (156). The pro-fibrotic effects require thiol/disulfide interaction as conjugating thiols with chemical reagents blocks fibronectin induction (156). In bleomycin-induced lung fibrosis mouse model, oxidation of plasma $\mathrm{Eh}_{\mathrm{h}} \mathrm{GSH} / \mathrm{GSSG}$ ) coincides with the pro-inflammatory phase while oxidation of plasma $E_{h}$ (Cys/CySS) occurs during the fibrotic phase (57). ELF $\mathrm{E}_{h}$ (Cys/CySS) is oxidized to a greater degree than plasma

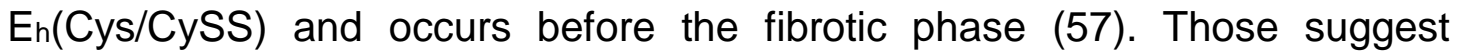
oxidation of redox couples might precede appearance of the fibrotic phenotype and represent a predisposing state with increased susceptibility to injuries from internal and external detrimental exposures.

Oxidative stress increases with aging and is considered an age-related defect. Aging is the most striking risk factor for IPF, and indicators of redox imbalance are observed in and contribute to IPF. Oxidation of redox couples accompanies aging in human plasma. Oxidation of redox couples also regulates multiple signaling pathways in vitro, including stimulation of pro- 
fibrotic cellular responses in mouse lung fibroblasts, and precedes bleomycininduced lung fibrosis in vivo. The downstream effects of oxidized redox potential have been explored while the upstream mechanism of whether and how redox potential are regulated remains unknown. Following study reveals the relationship between redox couples and mouse lung fibroblasts is reciprocal. Fibroblasts actively regulate their extracellular $E_{h}$ (Cys/CySS) in culture (157). A set-point value is reached and maintained for both fibroblasts from young and old mice regardless of whether the initial incubating media are oxidizing or reducing (157). The only difference between young and old fibroblasts is that the set-point value is more oxidizing for old fibroblasts, which is consistent with increased susceptibility of pro-fibrotic responses (157).

Slc7a11 expression is lower in old fibroblasts compared to young fibroblasts (157). It is the light chain subunit of a membrane-bound CySS/glutamate antiporter system $X_{c^{-}}{ }^{-}(158)$. System $X_{c^{-}}$imports CySS and exports glutamate with 1:1 exchange ratio. Inside the cells, CySS can be reduced to Cys, which is the rate-limiting component of GSH synthesis. Expression of SIc3a2, which is the other subunit of system $\mathrm{X}_{c}^{-}$and also known as $4 \mathrm{~F} 2$ heavy chain (4F2hc) or CD98, is not different between young and old fibroblasts $(157,159,160)$. Therefore, Slc7a11 might determine the activity of system $\mathrm{X}_{c}{ }^{-}$and is considered to be a candidate to regulate extracellular $E_{h}($ Cys/CySS) in primary mouse lung fibroblasts.

1.4 Specific aims 
IPF develops insidiously. Honeycombing involves destruction of basement membrane and is not reversible. By the time patients are diagnosed, extensive fibrosis characterized by widespread fibrotic foci and honeycombing has already formed, there is no available treatment to reverse the fibrosis. Therefore, identifying subclinical stage of IPF will probably provide patients with the utmost benefits. Since change of redox potential might precede detectable lung fibrosis, it is critical to understand how redox potential is regulated and whether redox potential is targetable to modify extracellular and intracellular redox environments so that lung redox homeostasis is restored, and the susceptibility of fibrosis is not increased.

This dissertation aims to investigate whether Slc7a11 mediates agedependent change of extracellular and intracellular redox potentials and how Slc7a11 affects intracellular signaling pathways in primary mouse lung fibroblasts. Redox potentials between fibroblasts from IPF patients and nonIPF donors will be compared and the role of Slc7a11 in human fibroblasts will also be explored.

Aim 1: To determine whether age-dependent Slc7a11 expression controls extracellular $E_{h}$ (Cys/CySS) in primary mouse lung fibroblasts.

My data indicate that Slc7a11 can be a potential controller of extracellular $E_{h}($ Cys/CySS) and therefore a switch of pro-fibrotic responses in fibroblasts. The experiments in Aim 1 will be composed of pharmacological and genetic manipulations of Slc7a11 expression to validate the regulatory role of Slc7a11. 
Whether Slc7a11 controls the balance of Cys/CySS and GSH/GSSG redox couples and their redox potentials extracellularly and intracellularly will be explored. The efficacy of sulforaphane to induce Slc7a11 will be demonstrated to establish the potentiality of sulforaphane as a drug to restore redox homeostasis in further clinical trials.

Aim 2: To uncover which protein cysteines and pathways are changed with aging and responsive to Slc7a11-dependent restoration of intracellular redox environment in primary mouse lung fibroblasts.

Aging changes intracellular redox environment and protein oxidation. However, whether Slc7a11 affects intracellular redox environment, to what degree Slc7a11 reverses the effects of aging on intracellular redox environment, and what responses Slc7a11-dependent change of redox environment induce in fibroblasts remain unknown. Thiols of cysteine residues in multiple proteins are targets of various redox modifications. Change of cysteine redox state, which is defined as the ratio of oxidized to reduced form of a specific cysteine thiol, might determine the activity of the cysteine-containing protein and affect downstream signaling pathways. Redox proteomics will be applied in Aim 2 to identify these intracellular protein cysteines whose redox states are altered with aging and in response to Slc7a11 manipulation. Biological processes and functions those proteins are involved in will be analyzed to elucidate agedependent and SIc7a11-sensitive signaling pathways in fibroblasts.

Aim 3: To compare SLC7A11 and redox environments in human lung 
fibroblasts from non-IPF donors and IPF patients.

The purpose of Aim 3 will be to demonstrate that the mechanisms explored in the first 2 Aims are directly applicable to humans. Primary human lung fibroblasts from patients with IPF and from non-IPF controls will be obtained. SLC7A11 expression, extracellular Eh(Cys/CySS), intracellular Eh(GSH/GSSG) and expression of pro-fibrotic genes will be measured in these human lung fibroblasts. This Aim will show the similarity between mouse and human lung fibroblasts and serve as an intermediate step between animal experiments and clinical trials. 


\title{
CHAPTER II
}

\author{
AGE-DEPENDENT OXIDATION OF EXTRACELLULAR \\ CYSTEINE/CYSTINE REDOX STATE (EH(CYS/CYSS)) IN MOUSE LUNG \\ FIBROBLASTS IS MEDIATED BY A DECLINE IN SLC7A11 EXPRESSION
}

\subsection{Introduction}

Reversible reduction and oxidation (redox) of the sulfur-containing amino acid cysteine (Cys) is exploited for a large number of biological processes (87). Redox reactive Cys can be found as the free amino acid, as part of the thiol antioxidant glutathione ( $\mathrm{\gamma}$-glutamylcysteinylglycine; $\mathrm{GSH})$, or as functional/regulatory sites within proteins (143). Cys and its oxidized form, cystine (CySS), constitute a redox couple that can be expressed in terms of its redox potential, or $E_{h}$ value. Likewise, GSH and its disulfide form, abbreviated GSSG, comprise another redox couple. These 2 couples are functionally connected but differentially regulated (127). Cys and CySS are present in greater concentrations than GSH and GSSG outside of cells, whereas GSH and GSSG predominate within the intracellular compartment $(161,162)$. In addition, each couple and each compartment are maintained at different redox potentials (147). Therefore, it is important to specify which compartment is 
being considered when reporting redox potentials. Both intracellularly and extracellularly Cys/CySS and GSH/GSSG function as redox buffers to maintain redox homeostasis (143) and resist or facilitate oxidation of protein thiols to change protein functions and transduce signals $(163,164)$. Thus, changes in redox potential can have a dramatic effect on cellular function. For example, oxidation of extracellular $\mathrm{E}_{h}$ (Cys/CySS) suppressed proliferation and inhibited signal transduction in Caco2 cells $(155,165)$, increased pro-inflammatory IL-1 $\beta$ in human monocytic U937 cells (166), and stimulated proliferation and profibrotic gene expression in mouse lung fibroblasts (156).

Oxidation of the extracellular space is reflected in changes in plasma redox potentials. In vivo studies have shown that plasma $\mathrm{Eh}_{\mathrm{h}}(\mathrm{Cys} / \mathrm{CySS})$ was oxidized in mice with bleomycin-induced lung injury (57), and in rats with kainic acid and pilocarpine-induced epilepsy (167). In humans, plasma $E_{h}$ (Cys/CySS) was found to be more oxidized in adults chronically exposed to arsenic (168), adults acutely exposed to acetaminophen (169), and in children with autism (170). Thus, oxidation of the extracellular environment, or redox stress, is associated with disease processes and environmental or pharmacological exposures.

Aging is a risk factor for development of a number of chronic diseases. One way in which aging may promote disease development or progression is by changing the set-point of the redox buffering systems. Aging is associated with a steady oxidation of plasma $\mathrm{E}_{h}$ (Cys/CySS) (136), but the mechanisms responsible are unclear. Cells in culture maintain an $\mathrm{E}_{h}(\mathrm{Cys} / \mathrm{CySS})$ remarkably 
close to the redox potential of plasma $(145,155,171)$, suggesting that cells are actively involved in controlling their immediate extracellular redox environment. Previous study showed that lung fibroblasts from old mice (24 months old) produced an extracellular $\mathrm{E}_{\mathrm{h}}$ (Cys/CySS) that was more oxidized than that produce by their young counterparts (2 months old) (157).

Differential gene expression analysis revealed that Slc7a11 was downregulated in old mouse lung fibroblasts (157). Slc7a11 (also called xCT) is the light chain of system Xc- which transports CySS into cells and exports glutamate with $1: 1$ as the exchange ratio (172). Previous studies have suggested that Slc7a11 expression is linked to control of the extracellular Cys/CySS redox state. Mice lacking Slc7a11 have a more-oxidizing extracellular $E_{h}(C y s / C y S S)$, as evidenced by an increase in their plasma CySS concentrations that is not balanced by a corresponding increase in plasma Cys (173). Conversely, stimulation of B cell differentiation is accompanied by an upregulation of Slc7a11 and an increase in extracellular Cys concentration (174). In the latter study there was also an increase in intracellular GSH, consistent with other studies showing that Slc7a11 activity supports intracellular GSH levels by supplying Cys, which is the rate-limiting amino acid for its synthesis (175). The purpose of the present study was to determine whether down-regulation of Slc7a11 in fibroblasts from old mice was sufficient to explain the oxidation of the extracellular redox environment associated with aging, and to determine whether synthesis of intracellular GSH was a pre-requisite for this 
effect.

2.2 Materials and methods

\subsubsection{Reagents}

Reagents were purchased from Sigma-Aldrich (St. Louis, MO) or Corning (Manassas, VA) unless otherwise specified.

2.2.2 Primary lung fibroblasts culture

Lung fibroblasts were isolated from young (3 months) or old (24 months) female C57BL/6 mice (157). Mouse lungs were harvested and cut into $1 \mathrm{~mm}$ sections (176). Those lung sections were washed with sterile phosphatebuffered saline (PBS) and resuspended in RPMI-1640 with L-glutamine containing $10 \%$ fetal bovine serum (FBS) and $1 \%$ antibiotic-antimycotic solution (176). Those tissues were transferred to cell culture dishes and incubated in a humidified $5 \% \mathrm{CO}_{2}$ incubator at $37^{\circ} \mathrm{C}$ for 7 to 21 days to let fibroblasts migrate out of tissues (176). Animal use was approved by the Institutional Animal Care and Use Committee of the University of Louisville. DMEM with $10 \%$ FBS and $1 \%$ antibiotic-antimycotic solution were used for regular cell culture (176). Fibroblasts between passage numbers 8 and 15 were used in the experiments.

\subsubsection{Preparation of redox media}

Redox media refers to media with specific Cys and CySS concentrations to achieve a specific redox state. DMEM without Met or Cys was used to make redox media. For $0 \mathrm{mV}$ (oxidizing) media, $99.75 \mu \mathrm{M}$ CySS and $0.5 \mu \mathrm{M}$ Cys were added. All redox media were freshly prepared immediately before use. 


\subsubsection{Pharmacological treatment of fibroblasts}

For sulforaphane experiments, primary lung fibroblasts from old mice were seeded in 6-well plates at the density of $1,000,000$ cells/well in DMEM containing $10 \%$ FBS and $1 \%$ antibiotic-antimycotic solution. After 24 hours, the media was changed in the induction group to fresh DMEM with $5 \mu \mathrm{M}$ sulforaphane. In the controls, media was changed to fresh DMEM. After incubation for 4 hours, media was changed to $0 \mathrm{mV}$ redox media and incubated for another 24 hours. Afterwards, media was collected for HPLC analysis and cells for qPCR analysis.

For sulfasalazine experiments, young fibroblasts were used. Twenty four hours after plating, media were changed to $0 \mathrm{mV}$ redox media with or without $300 \mu \mathrm{M}$ sulfasalazine. Four hours later, cells and media were collected.

For L-buthionine sulfoximine experiments, young fibroblasts were incubated with or without $20 \mu \mathrm{M}$ L-buthionine sulfoximine for 24 hours followed by 4 hours $0 \mathrm{mV}$ redox media incubation. Afterwards, cells and media were collected.

\subsubsection{Genetic manipulation of SIc7a11 expression in lung fibroblasts}

Plasmid transfection was used to over-express Slc7a11 in old fibroblasts, while siRNA was used to knock down Slc7a11 in young fibroblasts. Plasmid encoding mouse Slc7a11 was from Origene Technologies, Inc. (Rockville, MD), and siRNA was from Dharmacon (Lafayette, CO). Plasmid LacZ encoding for beta-D-galactosidase and non-targeted NT2 were used as plasmid and si-RNA 
control. Two $\mu \mathrm{g}$ of plasmid and $30 \mathrm{pmol}$ of siRNA were used for electroporation. Electroporation was conducted using Mode U-023 in Nucleofector ${ }^{\mathrm{TM}} 2 \mathrm{~b}$ Device (Lonza, Allendale, $\mathrm{NJ}$ ) following the protocol in the Amaxa ${ }^{\mathrm{TM}}$ Basic Nucleofector ${ }^{\mathrm{TM}}$ Kit for Primary Mammalian Fibroblasts (Lonza, Allendale, NJ). One million fibroblasts were used for each electroporation and then plated in 6well plates. Serum-free and antibiotic-free DMEM were used to incubate the transfected cells. After 24 hours recovery, DMEM were changed to $0 \mathrm{mV}$ media for 4 hours incubation. Then, media and cells were collected.

\subsubsection{Media derivatization and HPLC analysis}

Collected media were centrifuged at $800 \mathrm{~g}$ for 4 minutes to pellet the suspended fibroblasts. Five hundred $\mu \mathrm{l}$ cell-free media were combined with 500 $\mu \mathrm{l}$ ice-cold $10 \%(\mathrm{w} / \mathrm{v})$ perchloric acid (PCA), $0.2 \mathrm{M}$ boric acid and $20 \mu \mathrm{M} \mathrm{Y}$ glutamyl glutamate (177). These samples were derivatized by iodoacetic acid and dansyl chloride, and then analyzed by HPLC (Waters Corporation, Millford, MA) (178). For derivatization, $300 \mu$ of the media-PCA mixture were combined with $60 \mu$ of freshly prepared $14.8 \mathrm{mg} / \mathrm{ml}$ sodium iodoacetate. Then, the $\mathrm{pH}$ of the solution was adjusted to $9.0 \pm 0.2$ with $1 \mathrm{M}$ potassium hydroxide. The sample was incubated at room temperature for 20 minutes and mixed with 300 $\mu \mathrm{l}$ of freshly prepared $20 \mathrm{mg} / \mathrm{ml}$ acetone-dissolved dansyl chloride. After that, the sample was vortexed and incubated in the dark at room temperature for 24 hours. On the next day, $500 \mu \mathrm{l}$ of chloroform were added and the solution underwent vortex for 10 seconds. The top aqueous layer was used for 
concentration measurements by HPLC. Concentrations of Cys, CySS, GSH, GSSG, CySSG were measured by integration relative to the internal standard Y-glutamyl glutamate. Total Cys concentration $=[$ Cys $]+2 *[$ CySS $]+[$ CySSG $]$ and total GSH concentration $=[$ GSH $]+2^{*}[$ GSSG $]+[$ CySSG $]$. Eh of Cys $/$ CySS and GSH/GSSG were calculated according to the Nernst equation for $\mathrm{pH} 7.4$ : $E_{h}($ Cys $/$ CySS $)=-250+30^{*} \log \left([\right.$ CySS $\left.] /[\text { Cys }]^{2}\right) ; E_{h}($ GSH $/$ GSSG $)=-$ $264+30^{*} \log \left([G S S G] /[G S H]^{2}\right)(179)$.

2.2.7 Measurement of Slc7a11, Gclc and Nqo1 mRNA level

RNAqueous ${ }^{\circledR}-4$ PCR Kit (Thermo Fisher Scientific, Waltham, MA) was used for DNA-free RNA isolation from primary mouse lung fibroblasts. SuperScript ${ }^{\mathrm{TM}}$ VILO $^{\text {TM }}$ cDNA Synthesis Kit (Thermo Fisher Scientific, Waltham, MA) was used for cDNA synthesis. Real-time quantitative PCR (qPCR) was conducted to measure Slc7a11, Gclc, Nqo1 and Gapdh mRNA expression with TaqMan probes (TaqMan® Gene Expression Assay Mm00442530_m1, Mm00802655_m1, Mm01253561_m1, Mm99999915_g1; Applied Biosystems), according to the manufacturer's protocol (TaqMan Universal Master Mix II; Applied Biosystems). Step One Plus Real Time PCR System (Applied Biosystems) was used for qPCR with the parameters: $50^{\circ} \mathrm{C} 2$ minutes, $95^{\circ} \mathrm{C}$ 10 minutes, followed by 40 cycles of $95^{\circ} \mathrm{C} 15$ seconds and $60^{\circ} \mathrm{C} 1$ minute. Results were analyzed using Step One Software version 2.3 (Applied Biosystems). The amplification curves were analyzed by the mathematical equation of the second derivative, and the amounts of Slc7a11, Gclc, Nqo1 
mRNA expression were normalized to the housekeeping gene Gapdh mRNA expression. The $2^{-\Delta \Delta C T}$ method was used to calculate relative quantification (180).

\subsubsection{Measurement of Slc7a11 activity}

Slc7a11 activity was measured as the rate of glutamate release. Fresh 0 $\mathrm{mV}$ media containing $100 \mu \mathrm{M}$ CySS and $0 \mu \mathrm{M}$ glutamate were added to cells, and aliquots of conditioned media were removed at 15-minute intervals. Glutamate concentrations in media were measured using the Glutamate/Glutamate Oxidase assay kit from Molecular Probes (Waltham, MA) according to manufacturer's recommendations. L-glutamate was oxidized by glutamate oxidase to produce $\alpha$-ketoglutarate, ammonia and hydrogen peroxide. L-alanine and L-glutamate-pyruvate transaminase were used to regenerate L-glutamate by transamination of $\alpha$-ketoglutarate to amplify the signal. Large amounts of hydrogen peroxide were produced and reacted with the Amplex® Red reagent in a 1:1 stoichiometry to generate fluorescent resorufin that could be detected.

\subsubsection{Statistical analysis}

Data were presented as mean \pm standard deviation. Significance was evaluated by one-way ANOVA and unpaired two-tailed $t$-test. Linear regression was used to assess differences between rates of glutamate release.

\subsection{Results}

2.3.1 Manipulation of Slc7a11 by pharmacological agents 
Consistent with the previous studies (157), primary lung fibroblasts from old mice had lower expression of Slc7a11 (Figure 2.1.A) and more oxidized extracellular $E_{h}$ (Cys/CySS) redox potential (Figure 2.1.B) relative to fibroblasts from young mice. To begin to assess whether expression level of Slc7a11 was responsible for the observed differences in the extracellular redox states of young and old fibroblasts, old fibroblasts were treated with sulforaphane, an Nrf2 inducer known to increase expression of Slc7a11 (181). Sulforaphane increased Slc7a11 expression in old cells to the level seen in young cells (Figure 2.1.A). Sulforaphane also reduced extracellular $E_{h}(\mathrm{Cys} / \mathrm{CySS})$ to the value seen in young cells (Figure 2.1.B). There was no significant difference in the concentration of CySS in the conditioned media from cultures of young and old fibroblasts (Figure 2.1.C). Rather, a decrease in the amount of Cys (Figure 2.1.D) that accumulated in the media was responsible for the $30 \mathrm{mV}$ oxidation of old fibroblasts conditioned media. 
A

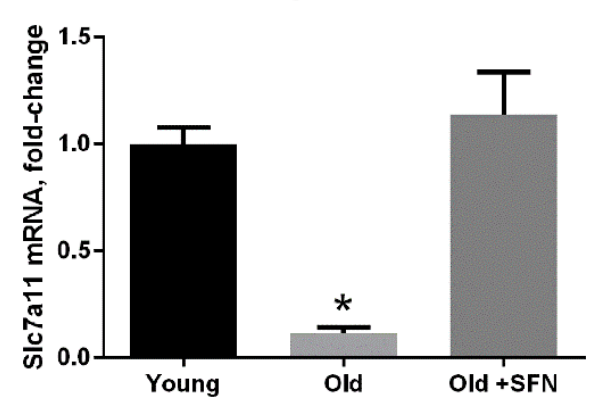

C

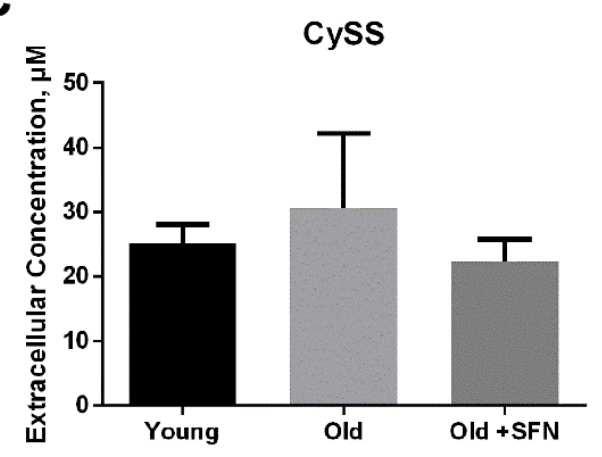

B $E_{h}$ (Cys/Cyss)

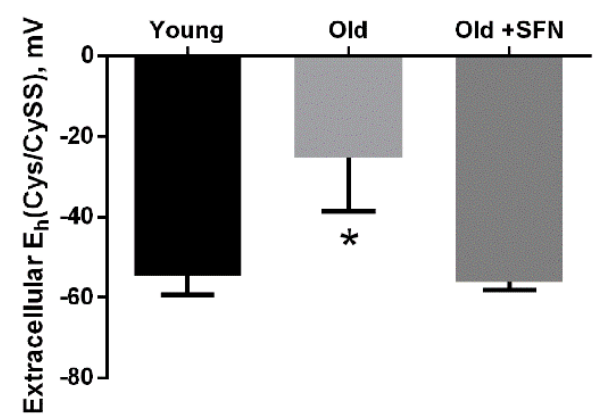

D

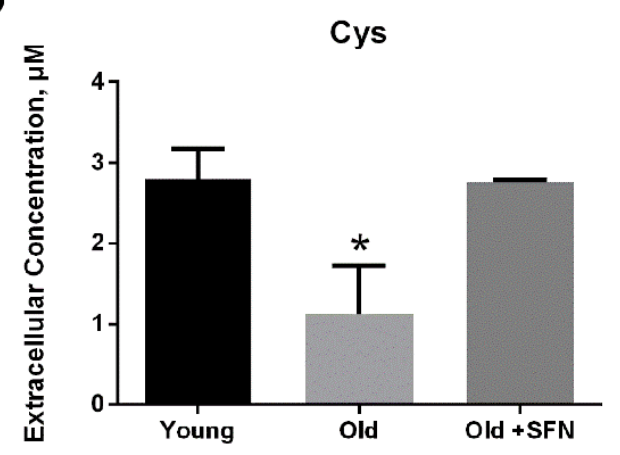

Figure 2.1 Sulforaphane treatment of old fibroblasts restored Slc7a11 expression and extracellular $E_{h}($ Cys/CySS) to the levels seen in young fibroblasts. Primary lung fibroblasts from young and old mice were incubated in DMEM with or without $5 \mu \mathrm{M}$ sulforaphane for 4 hours followed by 24 hours incubation in $0 \mathrm{mV}$ redox media. (A) Slc7a11 mRNA expression, (B) extracellular $\mathrm{Eh}_{\mathrm{h}}(\mathrm{Cys} / \mathrm{CySS}),(\mathrm{C})$ extracellular Cys concentration, and (D) extracellular CySS concentration, were measured as described in Materials and Methods. Data are expressed as mean \pm standard deviation of 3 independent replicates. * - Indicates $p<0.05$ compared to untreated young fibroblasts. 
Extracellular glutathione concentrations were also different between cultures of young and old fibroblasts. GSH, GSSG and the mixed disulfide between CySS and GSH (abbreviated CySSG) were all lower in the conditioned media from old cells compared to young cells (Figure 2.2.A). There was a larger decrease in GSH than in GSSG; as a result, the redox potential of this couple $(\mathrm{Eh}(\mathrm{GSH} / \mathrm{GSSG}))$ was $30 \mathrm{mV}$ more oxidized in the old cultures than in the young (Figure 2.2.B). GSH was not present in the $0 \mathrm{mV}$ redox media initially. Therefore, the appearance of GSH indicated that it was released from the cells. To facilitate analysis of GSH export, the total GSH pool size was calculated by combining GSH contributed by all 3 forms: GSH, GSSG and CySSG. As shown in Figure 2.2.C, total GSH was 4-fold lower in old fibroblasts than in young fibroblasts. Sulforaphane treatment restored extracellular GSH, GSSG, CySSG, total GSH and $E_{h}(G S H / G S S G)$ in old cultures to the levels seen in cultures of young fibroblasts (Figure 2.2.A-C). 
A

CySSG, GSH, GSSG

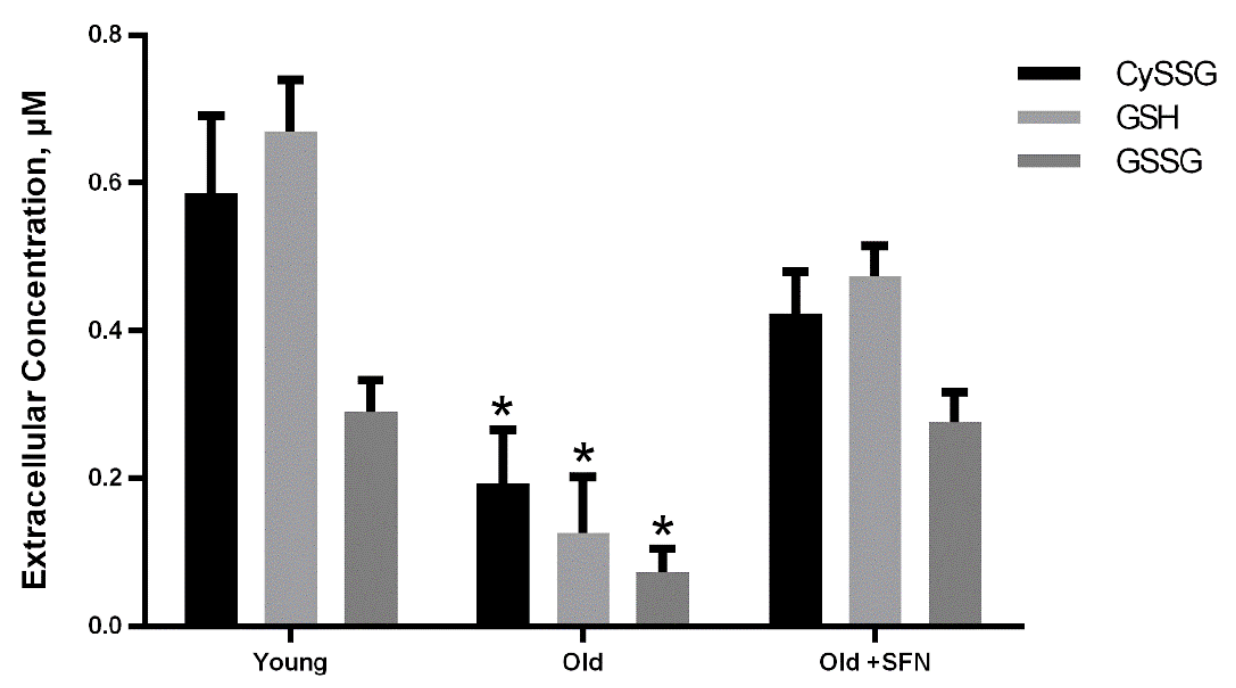

B

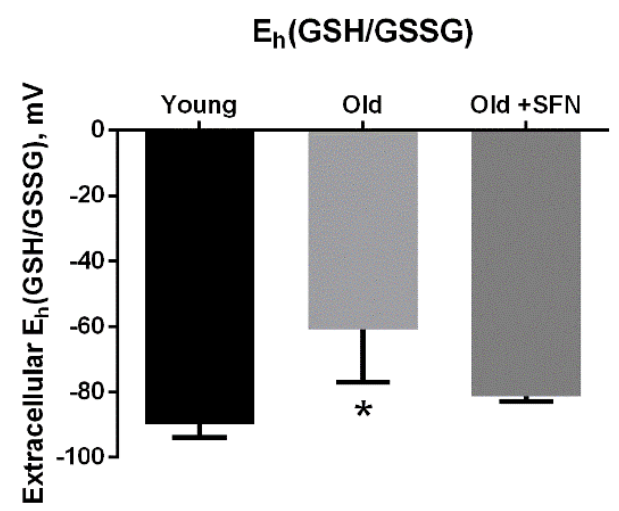

C

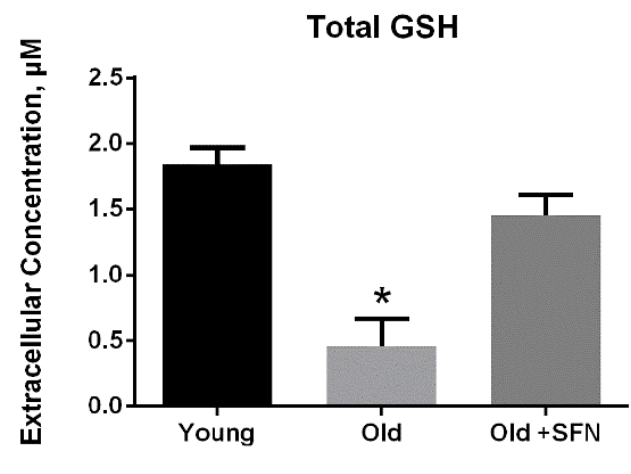

Figure 2.2 Glutathione was less abundant and more oxidized in the conditioned media of old fibroblasts, and sulforaphane corrected these deficiencies. Primary lung fibroblasts from young and old mice were incubated in DMEM with or without $5 \mu \mathrm{M}$ sulforaphane for 4 hours followed by 24 hours incubation in 0 $\mathrm{mV}$ redox media. (A) CySSG, GSH and GSSG, were measured in the conditioned medium by HPLC. (B) Extracellular Eh(GSH/GSSG) was calculated from the Nernst equation. (C) Total GSH concentration was calculated according to the formula: Total Cys $=$ CySSG + GSH $+2^{*} G S S G$. Data are expressed as mean \pm standard deviation of 3 independent replicates. ${ }^{*}$ - 
Indicates $p<0.05$ compared to untreated young fibroblasts. 
Sulforaphane is known to induce the expression of a number of antioxidant genes via its activation of the transcription factor Nrf2 (182). To determine the extent to which the effects of sulforaphane on extracellular $E_{h}$ (Cys/CySS) are mediated by SIc7a11 as opposed to its other transcriptional targets, cells were first transfected with siRNA to knock down Slc7a11 and then treated with sulforaphane. The results in Figure 2.3 showed that extracellular $E_{h}(C y s / C y S S)$ no longer became more reduced in response to sulforaphane in cells depleted of Slc7a11 (Figure 2.3.A). Expression of Slc7a11 was still induced by sulforaphane in the knock down cells, but not to the level seen in control cells (Figure 2.3.B). In contrast, other Nrf2 target genes (Gclc and Nqo1) were induced equally well in both control and knock down cells (Figures 2.3.C and 2.3.D). 
A

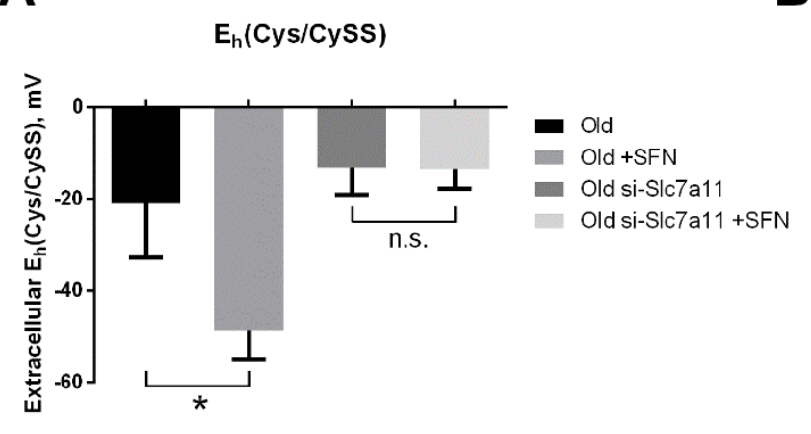

C

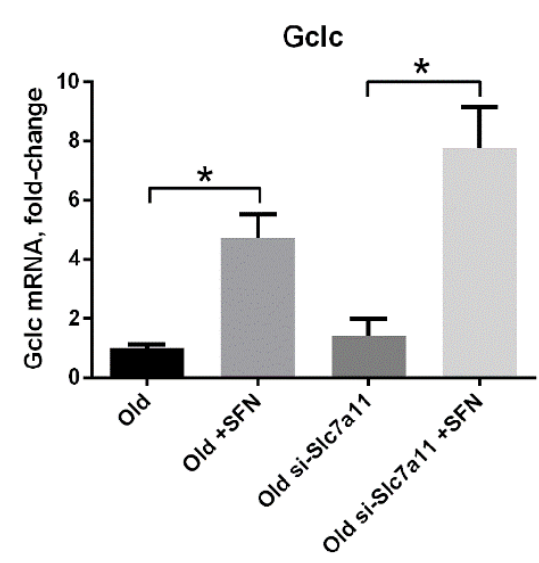

B

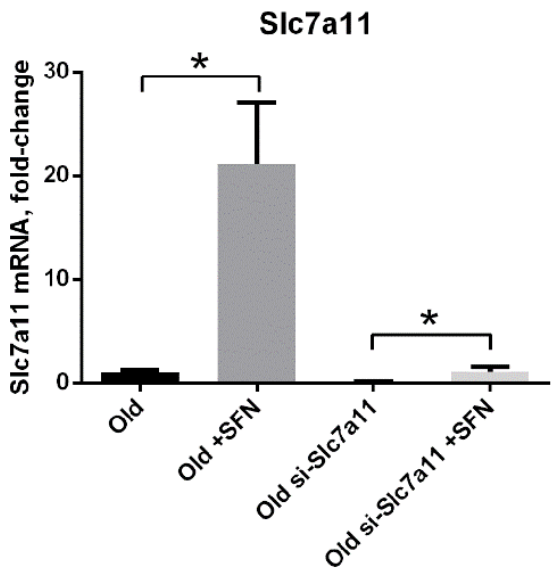

D

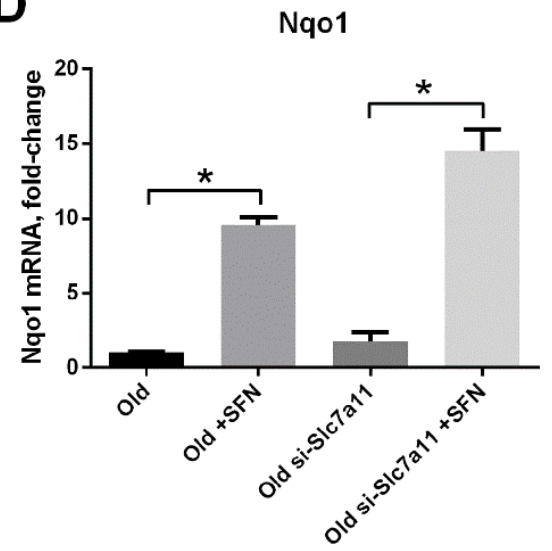

Figure 2.3 Reduction of extracellular $E_{h}($ Cys/CySS) in response to sulforaphane is mediated by Slc7a11. Primary lung fibroblasts from old mice were transfected with siRNA targeting Slc7a11 (si-Slc7a11) via electroporation. Non-targeting siRNA was electroporated as control. Fibroblasts were plated, and 24 hours later the media were changed to fresh DMEM with or without 5 $\mu \mathrm{M}$ sulforaphane for 4 hours. After that, media were changed to $0 \mathrm{mV}$ redox media for 4 hours. (A) Extracellular $E_{h}$ (Cys/CySS), (B) Slc7a11 mRNA expression, (C) Gclc mRNA expression, and (D) Nqo1 mRNA expression, were measured as described in the legends to Figures 2.1 and 2.2. Data are expressed as mean \pm standard deviation of 3 independent replicates. ${ }^{*}$ Indicates $\mathrm{p}<0.05$ between sulforaphane-treated and untreated fibroblasts. 
Having shown that a drug that increased Slc7a11 expression also reduced extracellular $\mathrm{En}_{\mathrm{h}}$ Cys/CySS), I sought to demonstrate that the inverse was also true: that inhibition of system Slc7a11 activity would oxidize extracellular Eh(Cys/CySS). For this purpose, sulfasalazine, an inhibitor of CySS transport that acts on the Slc7a11 subunit of system Xc-, was used(183). As predicted, sulfasalazine treatment of young fibroblasts oxidized extracellular Eh(Cys/CySS) by $30 \mathrm{mV}$ (Figure 2.4.A). This oxidation was due to a significant decrease in the concentration of extracellular Cys, while extracellular CySS concentration was unaffected by sulfasalazine (Figure 2.4.B and 2.4.C). Inhibition of Slc7a11 activity had no effect on accumulation of total GSH in the conditioned media (Figure 2.4.D). 
A

$E_{\mathrm{h}}$ (Cys/Cyss)

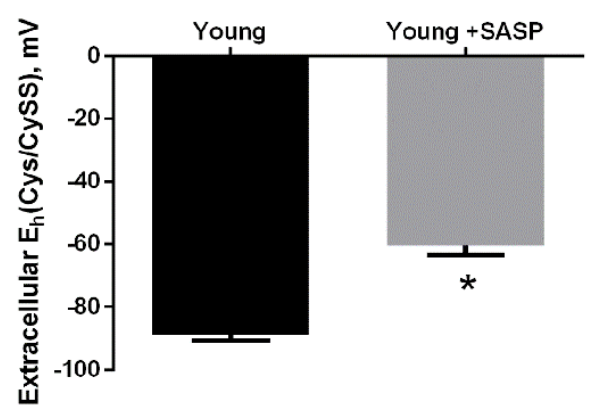

C

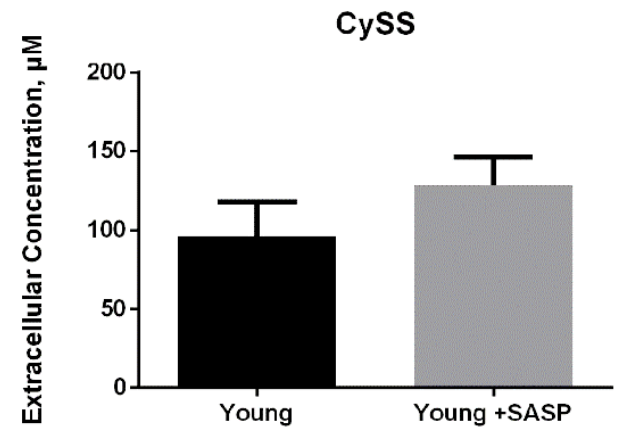

B

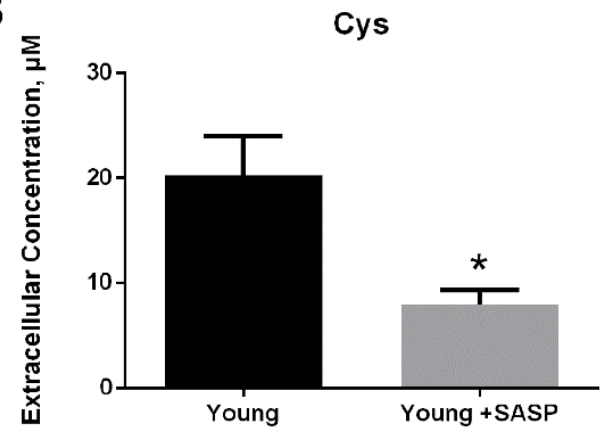

D

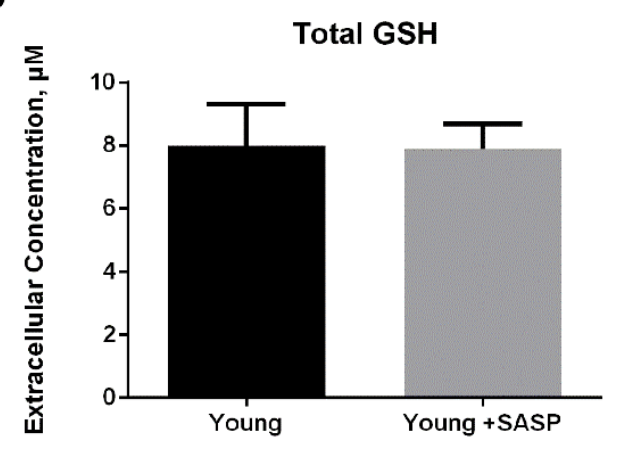

Figure 2.4 Slc7a11 inhibition by sulfasalazine in young mice lung fibroblasts resulted in oxidation of extracellular $\mathrm{E}_{h}(\mathrm{Cys} / \mathrm{CySS})$. Primary lung fibroblasts from young mice were incubated in $0 \mathrm{mV}$ redox media with or without $300 \mu \mathrm{M}$ sulfasalazine (SASP) for 4 hours. Conditioned media were collected for analysis by HPLC. (A) $E_{h}$ (Cys/CySS), (B) Cys concentration, (C) CySS concentration and (D) total GSH concentration were determined as described in the legends to Figures 2.1 and 2.2. Data are expressed as mean \pm standard deviation of 3 independent replicates. ${ }^{*}$ - Indicates $p<0.05$ compared to untreated young fibroblasts. 
Not only Slc7a11 mRNA, but also the activity of SIc7a11-mediated transport was lower in fibroblasts from old mice (Figure 2.5). Because Slc7a11 exports a glutamate for every CySS it imports, accumulation of glutamate in the media was measured as a function of time to assess transport activity. This approach avoided having to account for the multiple fates of CySS once imported into the cell. Using this approach, the pharmacological manipulations were demonstrated to translate to changes in activity. Sulforaphane increased Slc7a11 transporter activity (Figure 2.5.A), whereas sulfasalazine inhibited activity (Figure 2.5.B). 
A

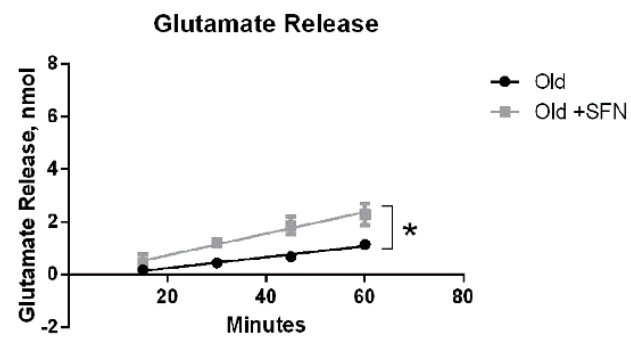

B

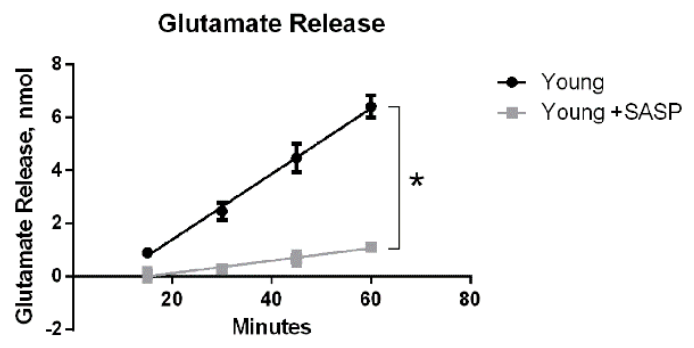

Figure 2.5 Slc7a11 transport activity was increased by sulforaphane and inhibited by sulfasalazine. (A) Glutamate release by old fibroblasts with or without sulforaphane treatment. Primary lung fibroblasts from old mice were incubated in DMEM with or without $5 \mu \mathrm{M}$ sulforaphane for 4 hours followed by 20 hours incubation in $0 \mathrm{mV}$ redox media. After that, media were changed to fresh $0 \mathrm{mV}$ media, and media was collected at $15 \mathrm{~min}, 30 \mathrm{~min}, 45 \mathrm{~min}$ and 60 min for measuring extracellular glutamate as described in Materials and Methods. (B) Glutamate release by young fibroblasts with or without sulfasalazine treatment. Primary lung fibroblasts from young mice were incubated in $0 \mathrm{mV}$ media with or without $300 \mu \mathrm{M}$ sulfasalazine. Media were collected at $15 \mathrm{~min}, 30 \mathrm{~min}, 45 \mathrm{~min}$ and $60 \mathrm{~min}$ for measuring extracellular glutamate. Data are expressed as mean \pm standard deviation of 3 independent replicates. ${ }^{*}$ - Indicates $p<0.05$ between treated and untreated fibroblasts. 
2.3.2 Modulation of Slc7a11 expression via genetic methods

Because pharmacological agents can have off-target effects, I next attempted to verify the above results using transfection to overexpress and knock down Slc7a11 in old and young fibroblasts. Similar to the results with induction by sulforaphane, transfection of old fibroblasts with a Slc7a11encoding plasmid increased Slc7a11 mRNA expression by three-fold (Figure 2.6. A) and reduced the extracellular $\mathrm{E}_{h}$ (Cys/CySS) by $30 \mathrm{mV}$ (Figure 2.6.B). Conversely, siRNA-mediated knock down of Slc7a11 in young fibroblasts decreased Slc7a11 mRNA level by three-fold (Figure 2.6.C). Correspondingly, extracellular $\mathrm{E}_{h}$ (Cys/CySS) became $45 \mathrm{mV}$ more oxidized after Slc7a11 knockdown (Figure 2.6.D). The changes in extracellular Eh(Cys/CySS) were largely driven by differences in Cys concentrations. Neither overexpression nor knock down had a significant effect on extracellular CySS concentrations (Figure 2.7.A and 2.7.B). However, Slc7a11 over-expression in old cells significantly increased extracellular Cys from $2 \mu \mathrm{M}$ to $5 \mu \mathrm{M}$ while Slc7a11 knock-down in young cells significantly decreased extracellular Cys from $6 \mu \mathrm{M}$ to $1 \mu \mathrm{M}$ (Figure 2.7.C and 2.7.D). Neither overexpression nor knock down of Slc7a11 had a significant effect on the accumulation of extracellular GSH (Figure 2.7.E and 2.7.F). 
A

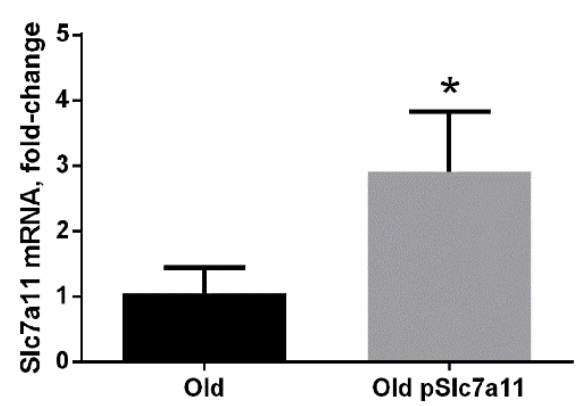

C

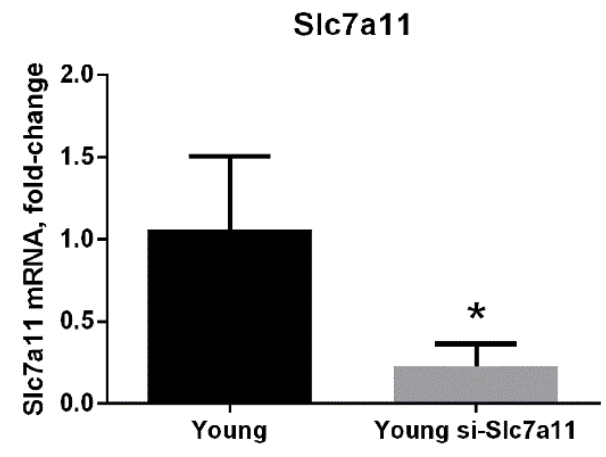

B $E_{h}($ Cys/Cyss)

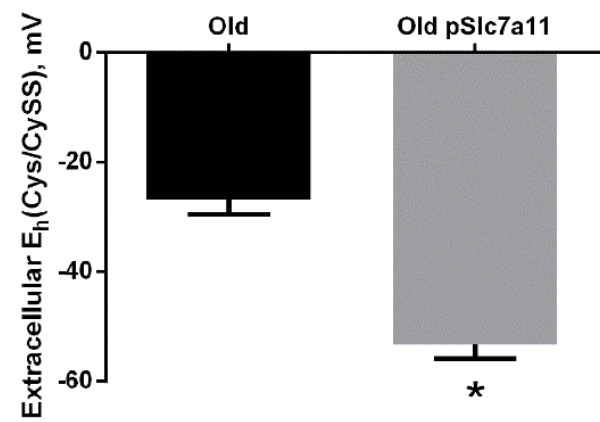

D

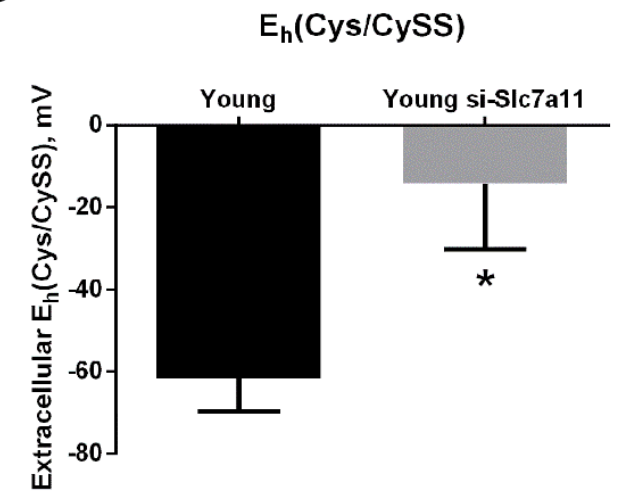

Figure 2.6 Effect of genetic manipulation of Slc7a11 on extracellular $\mathrm{E}_{h}$ (Cys/CySS). Primary lung fibroblasts from old and young mice were transfected with either an Slc7a11-encoding plasmid (pSlc7a11) or siRNA targeting Slc7a11 (si-Slc7a11) via electroporation. Controls were electroporated with pLacZ or non-targeting siRNA. Fibroblasts were plated, and 24 hours later the media were changed to $0 \mathrm{mV}$ redox media for 4 hours. (A) Slc7a11 mRNA expression and (B) extracellular $E_{h}($ Cys/CySS) in old fibroblasts with and without overexpression of Slc7a11. (C) Slc7a11 mRNA expression and (D) extracellular $E_{h}$ (Cys/CySS) of young fibroblasts with and without knock down of Slc7a11. Data are expressed as mean \pm standard deviation of 3 independent replicates. * - Indicates $p<0.05$ compared to controls. 
A

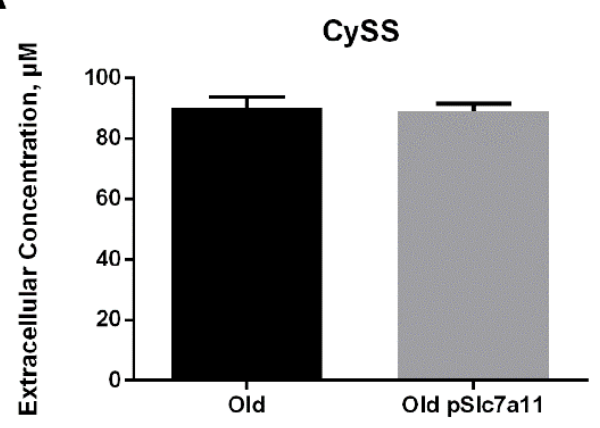

C

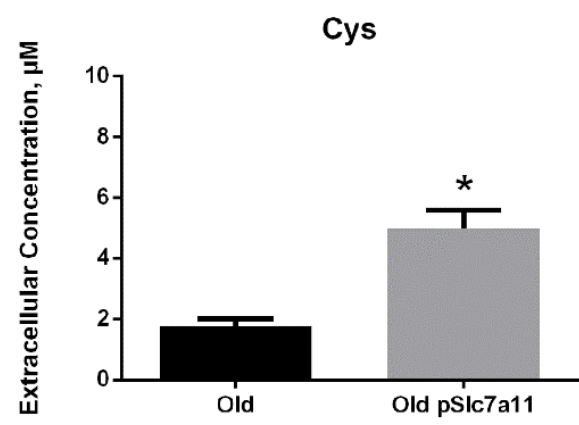

E

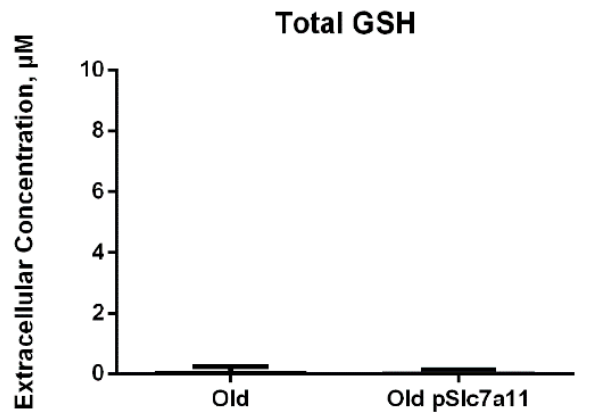

B

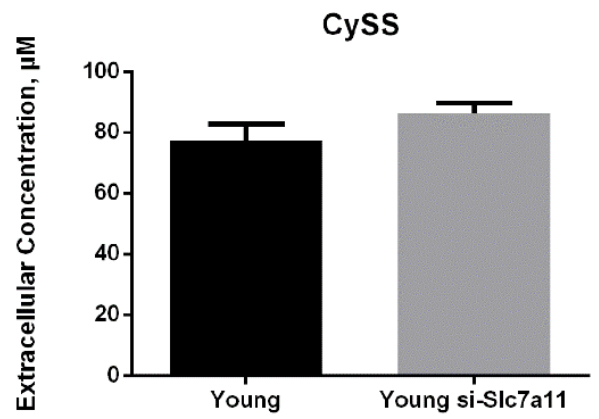

D

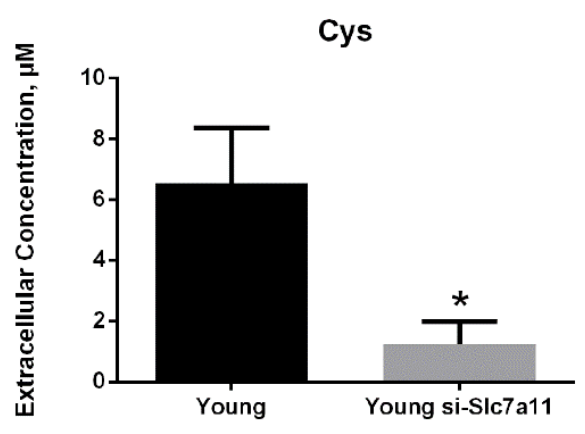

$\mathbf{F}$

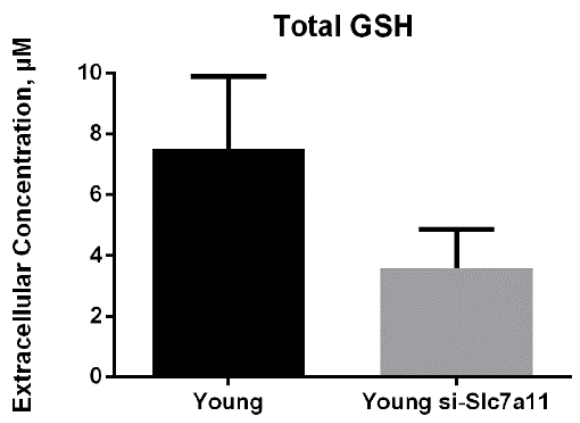

Figure 2.7 Overexpression of Slc7a11 increased, and knock down of Slc7a11 decreased, extracellular Cys concentrations. Primary lung fibroblasts from old and young mice were transfected with either pSIc7a11 (overexpression) or siSlc7a11 (knock down), as described in the legend to Figure 2.4. (A and B) Extracellular CySS concentrations, (C and D) extracellular Cys concentrations, and $(\mathrm{E}$ and $\mathrm{F})$ total extracellular $\mathrm{GSH}$ concentrations in old and young fibroblasts, respectively. Data are expressed as mean \pm standard deviation of 3 independent replicates. * - Indicates $p<0.05$ compared to controls. 
Both Cys and GSH can be exported by cells and used to reduce an oxidized extracellular $\mathrm{En}_{\mathrm{h}}$ Cys/CySS). To determine whether metabolism of Cys to GSH is necessary for cells to reduce an oxidizing extracellular $E_{h}(C y s / C y S S)$, buthionine sulfoximine (BSO) was used to inhibit GSH synthesis by $\mathrm{Y}^{-}$ glutamylcysteinyl ligase. As shown in Figure 2.8, 24 hours pre-treatment with BSO dramatically lowered intracellular GSH (Figure 2.8.A). The inhibition of GSH synthesis by BSO also led to a dramatic decrease in extracellular GSH concentrations (Figure 2.8.B). BSO treatment had no effect on extracellular Cys concentration (Figure 2.8.C) or the ability of cells to restore extracellular $\mathrm{E}_{\mathrm{h}}(\mathrm{Cys} / \mathrm{CySS})$ within 4 hours of challenge with $0 \mathrm{mV}$ media (Figure 2.8.D), suggesting that GSH synthesis was not required for the normalization of an oxidizing extracellular $E_{h}(C y s / C y S S)$. 
A

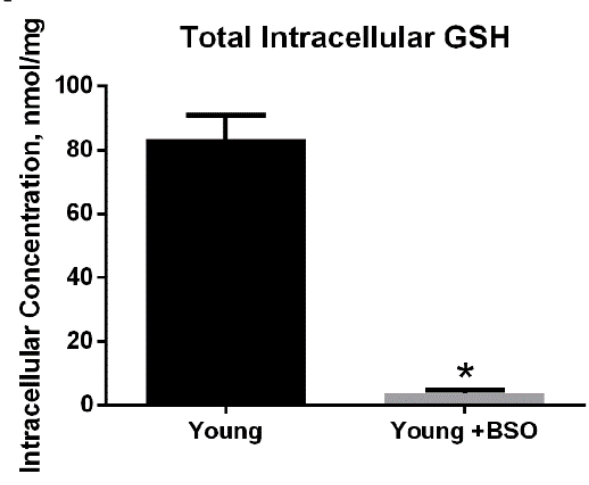

C

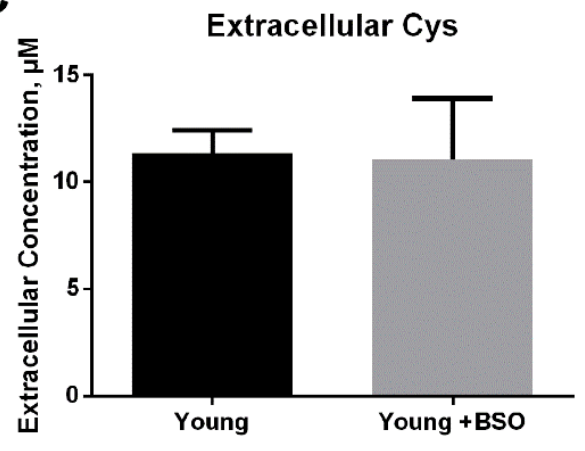

B

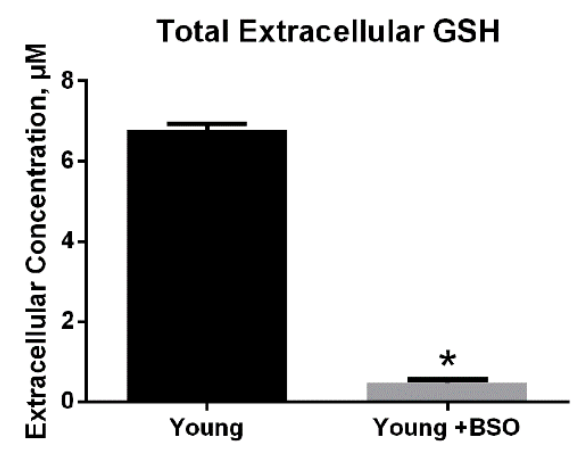

D

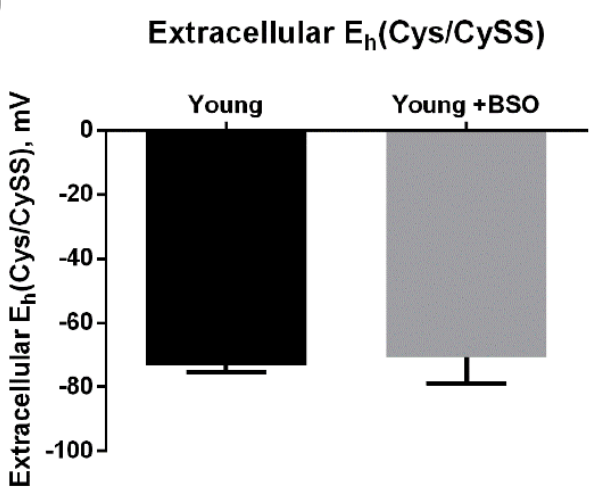

Figure 2.8 Glutathione depletion with BSO did not affect extracellular Cys concentration or $E_{h}$ (Cys/CySS). Primary lung fibroblasts from young mice were incubated in $0 \mathrm{mV}$ redox media with or without $20 \mu \mathrm{M}$ L-buthionine sulfoximine (BSO) for 24 hours. (A) Total intracellular GSH. (B) Total extracellular GSH. (C) Extracellular Cys concentration. (D) Extracellular $E_{h}$ (Cys/CySS). Data are expressed as mean \pm standard deviation of 3 independent replicates. * Indicates $p<0.05$ compared to controls. 


\subsection{Discussion}

The current studies confirm the finding that Slc7a11 expression was lower in primary lung fibroblasts from old mice than in those from young mice, and that this was associated with increased oxidation of extracellular $E_{h}($ Cys/CySS) redox potential (157). Those findings have now been extended by investigating the mechanistic link between these two observations. Up-regulation of Slc7a11 expression by either sulforaphane treatment or transient transfection was sufficient to restore the ability of lung fibroblasts from old mice to reduce their extracellular $E_{h}$ (Cys/CySS) to the level achieved by those from young mice. Conversely, inhibition of Slc7a11 activity by either sulfasalazine treatment or siRNA-mediated knock down produced young fibroblasts that resembled old fibroblasts in terms of their extracellular redox potential. Taken together, these findings show that Slc7a11 activity is the major determinant of the extracellular redox environment produced and maintained by primary lung fibroblasts.

In each of the studies presented here, Slc7a11 was positively correlated with extracellular Cys concentrations. This suggests that intracellular reduction of CySS to Cys, and the subsequent export of Cys, is limited by the rate of delivery of CySS to cells. Differences in Slc7a11 activity had no effect on extracellular CySS concentrations under the culture conditions used in this study. This is most likely a reflection of the process by which cells regulate their extracellular redox environment. CySS cannot be reduced to Cys in the extracellular space. Therefore, cells adjust the relative proportions of 
extracellular CySS and Cys by importing CySS, reducing it to Cys, and then exporting Cys via system alanine-serine-cysteine (system ASC) (184-187). To achieve physiological redox potential of about $-80 \mathrm{mV}$ (136), only a fraction of the media CySS needed to be reduced to Cys. Because each molecule of CySS yields 2 molecules of Cys, very little CySS is consumed in the process of normalizing the extracellular redox potential. Thus, CySS import via Slc7a11 appears to be the rate-limiting factor for intracellular Cys formation and export, just as it is rate-limiting for GSH synthesis in some cell types $(188,189)$.

An increase in extracellular Cys, but not CySS, in response to increased Slc7a11 activity has been observed previously. Overexpression of Slc7a11 in Burkitt's Lymphoma cells was associated with increased extracellular Cys concentrations (190), similar to my results. In contrast, knock out mice had elevated plasma CySS, but no change in plasma Cys (173). The discrepancy between these findings and my results with sulfasalazine or siRNA-mediated knock down of Slc7a11 may have been due to the fact that the fibroblasts used in the current studies do not completely lack Slc7a11, or it could be the presence of other cell types in the mice that may remove Cys from the plasma (191).

Of the 3 component amino acids of GSH, Cys is usually present at the lowest concentrations within cells and limits the rate at which GSH can by synthesized (175). Therefore, changes in Slc7a11 activity can affect intracellular GSH production. In cancer cells, elevated Slc7a11 expression is 
associated with increased resistance to chemotherapy drugs (192). Conversely, inhibition of CySS transport can sensitize cancer cells to radiation therapy (193). The age-related decrease in Slc7a11 expression observed in the current study may limit GSH synthesis and contribute to lower extracellular concentrations of GSH.

CySS and GSH have many fates both extracellularly and intracellularly, complicating efforts to account for contributions of metabolism and transport to changes in concentrations in any given location. Extracellular GSH can be used to increase extracellular Cys concentrations by two mechanisms: it can undergo thiol-disulfide exchange with extracellular CySS to yield Cys and the mixed disulfide CySSG, or it can be catabolized enzymatically by gammaglutamyltransferase and dipeptidase to yield Cys and the other two component amino acids of GSH, glutamate and glycine (175). GSH can also be oxidized to GSSG and used to glutathionylate extracellular proteins. Similarly, CySS can cysteinylate proteins. Extracellular Cys itself can be taken up by some cell types through other amino acid transporters such as systems ASC, EAAT and LAT2 $(194,195)$. Once CySS is imported, it can be reduced to Cys either nonenzymatically through thiol-disulfide exchange with GSH or enzymatically by Txnrd1 or Txndc17 (186). Intracellular Cys can be used to synthesize proteins or GSH, which can then by oxidized, glutathionylate proteins, conjugate to electrophilic metabolites, or be exported. Despite the complexity of these interconnected pathways, my data point to a rather straightforward relationship 
between Slc7a11 activity and extracellular $\mathrm{E}_{\mathrm{h}}$ (Cys/CySS). Importantly, GSH was not involved in regulation of extracellular $\mathrm{En}_{\mathrm{h}}(\mathrm{Cys} / \mathrm{CySS})$, and that accumulation of Cys in the extracellular space was directly related to the level of Slc7a11 activity.

Extracellular GSH was less abundant and more oxidized in cultures of lung fibroblasts from old mice. Whereas sulforaphane treatment corrected these defects, Slc7a11 overexpression did not. A likely explanation for this discrepancy is that sulforaphane activates a much broader antioxidant response than does Slc7a11 overexpression alone. For example, my study confirmed that, in addition to Slc7a11 induction, sulforaphane upregulated the expression of Gclc, the catalytic subunit of the rate-limiting enzyme in GSH synthesis. Both Slc7a11 and Gclc are transcriptionally regulated by the transcription factor Nrf2. Sulforaphane is an electrophile that interacts directly with nucleophilic residues on Keap1, thereby activating Nrf2 (196). In cancer cells, sulforaphane can induce anticancer responses driven in part through production of reactive oxygen species via interactions with mitochondrial respiratory complex I (197). Oxidative stress produced in this way can promote the formation of 4-hydroxynonenal, an endogenous electrophilic activator of Nrf2 (198). However, untransformed cells, such as the primary fibroblasts used in the present study, are typically protected from the anticancer effects of sulforaphane (199).

In conclusion, oxidative stress has been well recognized in aging. While 
oxidative stress can be measured in many ways, my studies focused on oxidation of the extracellular $\mathrm{E}_{\mathrm{h}}$ (Cys/CySS) redox potential. Oxidation of extracellular $\mathrm{E}_{\mathrm{h}}$ (Cys/CySS) has been linked to age-dependent lung matrix remodeling and changes in the phenotype of lung fibroblasts $(156,157)$, as well as phenotypic changes in other cell types $(144,200,201)$. Identification of Slc7a11 as a critical factor in the regulation of the extracellular redox environment will undoubtedly lead to novel approaches to understanding the effects of aging in health and disease. 
CHAPTER III

REDOX STATES OF PROTEIN CYSTEINES IN PATHWAYS OF PROTEIN TURNOVER AND CYTOSKELETON DYNAMICS ARE CHANGED WITH AGING AND REVERSED BY SLC7A11 RESTORATION IN MOUSE LUNG FIBROBLASTS

3.1 Introduction

Aging has been proposed as a consequence of failure of redox networks to sustain biological functions (202). This redox theory of aging accounts for several hallmarks of aging, including altered intercellular communication, loss of proteostasis, epigenetic alterations and mitochondrial dysfunction $(27,202)$, because each of these is sensitive to changes in redox state of one or more of its constituent components. One convenient way to assess changes in systemic redox states is to measure the redox potential $\left(E_{h}\right)$ of the cysteine/cystine (Cys/CySS) thiol/disulfide redox couple (Eh(Cys/CySS)). Human plasma typically has an $E_{h}($ Cys/CySS) of about $-80 \mathrm{mV}$, and cells grown in culture condition their media to this same value $(134,157)$. As we age, plasma $E_{h}$ (Cys/CySS) becomes progressively more oxidized (136), and cultured old cells condition their media to more oxidized values when compared to young 
cells (157). Thus, aging is associated with a disrupted redox environment and decreased ability to fight against oxidative stress.

Previous studies showed that the expression of Slc7a11, the light chain of the CySS transporter system $X_{c^{-}}$, was decreased in old mouse lung fibroblasts compared to that of young fibroblasts, concomitant with oxidation of extracellular $E_{h}($ Cys/CySS) that occurs with age $(157,203)$. Pharmacological induction and genetic overexpression of Slc7a11 restored extracellular balance of the Cys/CySS redox couple and its $E_{h}(\mathrm{Cys} / \mathrm{CySS})$ in a glutathione (GSH) synthesis-independent manner, confirming Slc7a11 is responsible for controlling extracellular $E_{h}($ Cys/CySS) in primary mouse lung fibroblasts (203). Previous studies also showed extracellular Eh(Cys/CySS) was associated with a variety of biological processes and diseases. For example, medium with oxidized $E_{h}($ Cys/CySS $)$ induced the expression of TGF- $\beta$ and fibronectin and stimulated the proliferation of mouse lung fibroblasts (156). Oxidized extracellular $E_{h}$ (Cys/CySS) also promoted mitochondrial thioredoxin-2 oxidation and induced reactive oxygen species (ROS) production in aortic endothelial cells (67). In C6 glial cells, oxidized $E_{h}(\mathrm{Cys} / \mathrm{CySS})$ increased phosphorylation of extracellular signal-regulated kinase (ERK) and expression of nuclear factor-kB (NF-kB) and inducible nitric oxide (iNOS) via activating metabotropic glutamate receptor 5 (153). Plasma $E_{h}$ (Cys/CySS) was more oxidized in patients with chronic obstructive pulmonary disease compared to that in patients with normal lung function (150). Thus, Slc7a11 has the potential 
to influence important intracellular signaling pathways and biological functions via controlling the extracellular redox environment.

Once transported into cells by system $X_{c}^{-}$, CySS is reduced to Cys, which is the rate-limiting component for GSH synthesis. GSH and Cys have the capacity to influence the redox states of individual intracellular proteins. Proteins contain cysteines that can be reversibly oxidized to disulfides or sulfenic acids. If a redox-sensitive cysteine is located in a catalytic or allosteric regulatory site, or a protein-protein or protein-DNA interaction domain, its redox state can determine the function of that protein. Many protein kinases have been shown to contain redox-sensitive cysteines. For example, oxidation of Cys797 to a sulfenic acid in the active site of EGFR increased its tyrosine kinase activity (204). In contrast, intramolecular disulfide bond between Cys297 and Cys311 and oxidation of Cys 124 to a sulfenic acid inhibited the kinase activity of Akt2, demonstrating that predicting specific changes of biological function resulting from alterations in the redox states of cysteines is difficult, thereby requiring evaluation on a case-by-case basis and requiring experimental confirmation $(205,206)$. Furthermore, redox changes can affect structural proteins that contain redox-reactive thiols. In primary mouse lung fibroblasts, for example, cadmium exposure induced oxidation of cysteines of actin and actin-associated cytoskeleton proteins, thus changing actin dynamics via increasing filamentous actin formation and transporting destrin from cytoplasm to nucleus (207). 
The large number and wide distribution of protein cysteines increase the functional flexibility of the proteome and play important roles in signal transduction, aging and disease (208). Redox proteomics can be used to define the redox states of all protein cysteines under a given set of conditions. Various redox proteomics methods have been developed, but they share some common features. In general, these methods rely on differentially labeling reduced and oxidized cysteines and separating the 2 forms from each other, followed by quantification of each form (209). This approach was recently used to study the change of redox states of proteins in brown adipose tissue of mice exposed to cold temperature (210). This method has been adapted for labelling reduced and oxidized cysteines to compare redox states between murine young and old lung fibroblasts, and old fibroblasts with Slc7a11 overexpression.

Currently, there is no information about how Slc7a11 affects the redox state and function of the cysteine proteome. Furthermore, how aging changes the redox state of the cysteine proteome in primary mouse lung fibroblasts is unknown. This study aims to identify intracellular protein cysteines whose redox states are age-dependent, and to explore the potential biological functions of these cysteine-containing proteins. Considering that low Slc7a11 expression in old fibroblasts is responsible for the change of the extracellular redox environment with aging, I also aim to explore whether and to what extent restoration of Slc7a11 expression would reverse the age-dependent changes of intracellular redox environment and signaling pathways. 
3.2 Materials and methods

\subsubsection{Reagents}

All reagents were purchased from Sigma-Aldrich (St. Louis, MO) or Corning (Manassas, VA) unless otherwise specified.

3.2.2 Culture of primary mouse lung fibroblasts

Primary lung fibroblasts were isolated from young (3 months) or old (24 months) female C57BL/6 mice as described in Chapter $2(176,211)$. Animal maintenance and procedures of animal experiments were approved by the Institutional Animal Care and Use Committee of the University of Louisville. After isolation, fibroblasts were cultured in DMEM supplemented with $10 \%$ fetal bovine serum (FBS) and 1\% antibiotic-antimycotic solution in a humidified incubator with $5 \% \mathrm{CO}_{2}$. Fibroblasts between passage numbers 10 and 15 were used in the experiments.

3.2.3 Preparation of redox media with $\mathrm{E}_{\mathrm{h}}$ (Cys/CySS) of $0 \mathrm{mV}$

$0 \mathrm{mV}$ redox media were prepared from adding CySS and Cys to cysteinefree and FBS-free DMEM to reach the final concentrations of $99.75 \mu \mathrm{M}$ for CySS and $0.5 \mu \mathrm{M}$ for Cys. All redox media were freshly prepared and used for fibroblast incubation immediately.

\subsubsection{Overexpression of Slc7a11 expression}

Plasmid encoding mouse Slc7a11 was purchased from Origene Technologies, Inc. (Rockville, MD). Two micrograms of plasmid were used to overexpress Slc7a11 in lung fibroblasts from old mice via electroporation. The 
program of U-023 on the Nucleofector ${ }^{\mathrm{TM}} 2 \mathrm{~b}$ Device (Lonza, Allendale, $\mathrm{NJ}$ ) and the protocol of the Amaxa ${ }^{\mathrm{TM}}$ Basic Nucleofector ${ }^{\mathrm{TM}}$ Kit for Primary Mammalian Fibroblasts (Lonza, Allendale, NJ) were applied for electroporation. One million fibroblasts underwent transfection and were seeded in 6-well plates. Fibroblasts were recovered in antibiotic-free DMEM with 10\% FBS. After 24 hours, the media were changed to $0 \mathrm{mV}$ redox media for 4 hours incubation. Finally, media and cells were collected for measuring extracellular $\mathrm{Eh}$ (Cys/CySS) via HPLC and redox states of cysteine residues of intracellular proteins, respectively.

3.2.5 Media derivatization and high-performance liquid chromatography (HPLC) analysis

Detailed procedures were described in Chapter 2 (203). Briefly, media were transferred to a tube containing the same amount of ice-cold solution consisting of $10 \%(\mathrm{w} / \mathrm{v})$ perchloric acid, $0.2 \mathrm{M}$ boric acid and $20 \mu \mathrm{M}$-glutamyl glutamate (177). Then, media were derivatized with iodoacetic acid and dansyl chloride for HPLC analysis (Waters Corporation, Millford, MA) (178). Calculation of $\mathrm{E}_{h}$ (Cys/CySS) was based on the Nernst equation for $\mathrm{pH} 7.4: \mathrm{E}_{h}(\mathrm{Cys} / \mathrm{CySS})=-$ $250+30^{*} \log \left([\right.$ CySS $\left.] /[\text { Cys }]^{2}\right)(134)$

3.2.6 Labeling of reduced and oxidized cysteine residues of intracellular proteins with different iodoacetyl tandem mass tags (iodoTMTs)

My labeling method was developed based on the isobaric iodoTMTsixplex reagent. Each iodoTMT label contains a thiolate specific-reactive group for 
labeling reduced thiols, a mass reporter with different numbers of isotope positions for distinguishing different tags in the mass spectrometry, and a mass normalizer for balancing the molecular weight. Proteins of fibroblasts in each well were collected and frozen in $300 \mu$ of $10 \%$ trichloroacetic acid (TCA) before labeling. After thawing, proteins were centrifuged at $16,000 \mathrm{~g}$ at $4{ }^{\circ} \mathrm{C}$ for $15 \mathrm{~min}$. The protein pellet was washed with ice-cold acetone. Then, the protein pellet was dissolved in $200 \mu \mathrm{l}$ of denaturing buffer consisting of $200 \mathrm{mM}$ Tris, $1 \mathrm{mM}$ EDTA and $0.1 \%(w / v)$ SDS, pH 8.5. Immediately thereafter, $100 \mu$ of dissolved proteins was added to a tube containing one of six iodoTMT reagents freshly dissolved in $10 \mu \mathrm{l}$ methanol and incubated for 1 hour at $37^{\circ} \mathrm{C}$. This step labels the reduced cysteines. Proteins were precipitated once again in TCA and washed with acetone to remove unincorporated iodoTMT reagent. Proteins were dissolved in $100 \mu \mathrm{l}$ of denaturing buffer and $1 \mu \mathrm{l}$ of $500 \mathrm{mM}$ TCEP was added to reduce reversibly oxidized protein cysteine residues. The newlyreduced cysteines were then labeled by adding the second iodoTMT label and incubating for 1 hour at $37^{\circ} \mathrm{C}$. Again, proteins were precipitated with TCA and washed with acetone to remove unincorporated iodoTMT. Finally, doublelabelled protein pellet was dissolved in $60 \mu \mathrm{l}$ of denaturing buffer.

3.2.7 Digestion, affinity purification and multiplexed liquid chromatographytandem mass spectrometry (LC-MS/MS)

Detailed procedures were described here according to a previous study (212). The same amount of labelled proteins from different groups were mixed. 
Then, trypsin was used to digest the proteins at $37^{\circ} \mathrm{C}$ overnight. After digestion, peptides were lyophilized and dissolved in $100 \mu \mathrm{l}$ of Tris-buffered saline (TBS). $0.2 \mathrm{ml}$ of anti-TMT resin beads ( $0.4 \mathrm{ml}$ of $50 \%$ slurry) was used for enrichment of the digested peptides. Peptides were incubated with the beads at $4{ }^{\circ} \mathrm{C}$ overnight. Then, the beads were washed 3 times with $200 \mu$ of TBS, followed by 3 times with $0.2 \mathrm{ml}$ water. Finally, the bound peptides were eluted 3 times with $0.2 \mathrm{ml}$ elution buffer. The elutes were then desalted with C18 spin column (Pierce, Rockford, IL), dried by speed vacuum, dissolved in $1 \%$ formic acid and analyzed by multiplexed LC-MS/MS.

\subsubsection{Ingenuity Pathway Analysis (IPA)}

Redox state of a cysteine residue in a protein was defined as the ratio of its oxidized form to reduced form. Peptides containing cysteine residues that had significant change of redox state with aging were selected. Among them, the redox states of cysteines in a subgroup of peptides were reversed by Slc7a11 overexpression. Proteins containing peptides in this subgroup were used for IPA (http://www.ingenuity.com) and bioinformatic analyses. IPA predicts networks, canonical pathways and molecular functions associated with the input list of genes or proteins (213). Fold-changes of the redox states of cysteines between old and young fibroblasts for the above subgroup were used as IPA input. The false discovery rate (FDR) threshold was set at 0.05 .

3.2.9 Gene Ontology (GO) enrichment and Kyoto Encyclopedia of Genes and Genomes (KEGG) pathway analysis 
GO analysis is used for annotating genes and their products from 3 categories, including biological process (BP), molecular function (MF) and cellular component (CC) (214). KEGG (www.genome.jp/kegg/) is a database for linking genomic information with functional information to achieve analysis of functions of genes systematically (215). Online Database for Annotation, Visualization and Integrated Discovery (DAVID, https://david.ncifcrf.gov/) was used to conduct GO enrichment analysis and KEGG pathway analysis (216). GO and KEGG terms with a $p$-value $<0.05$ were considered significantly enriched.

3.2.10 Protein-protein interaction (PPI) network construction

STRING database (http://www.string-db.org/) and Cytoscape 3.6.1 (https://cytoscape.org/) were used to construct PPI network to explore functional associations between proteins $(217,218)$. The minimum required interaction score was medium confidence $(\geq 0.4)$.

\subsection{Results}

3.3.1 Slc7a11 overexpression corrects extracellular $E_{h}$ (Cys/CySS) in old fibroblasts

My goal is to explore how aging affects redox states of intracellular cysteine residues of proteins, and to examine whether Slc7a11 overexpression in old cells reverses some, if not all, of those changes. Consistent with the previous studies in Chapter $2(157,203)$, primary lung fibroblasts from old mice showed lower Slc7a11 expression and more oxidized extracellular $E_{h}$ (Cys/CySS) 
compared to fibroblasts harvested from young mice (Figure 3.1). Genetic overexpression of Slc7a11 by plasmid in old fibroblasts successfully restored Slc7a11 expression and resulted in reduction of extracellular $E_{h}(\mathrm{Cys} / \mathrm{CySS})$ close to the levels seen in young fibroblasts, confirming the cell model for testing the effects of Slc7a11 on the redox environment had been reproduced (Figure 3.1). 


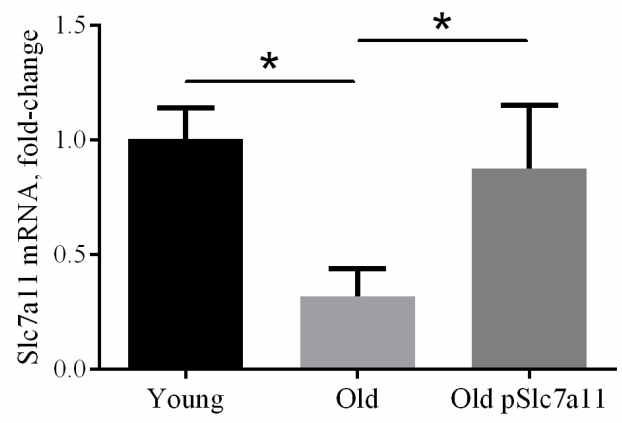

(a)

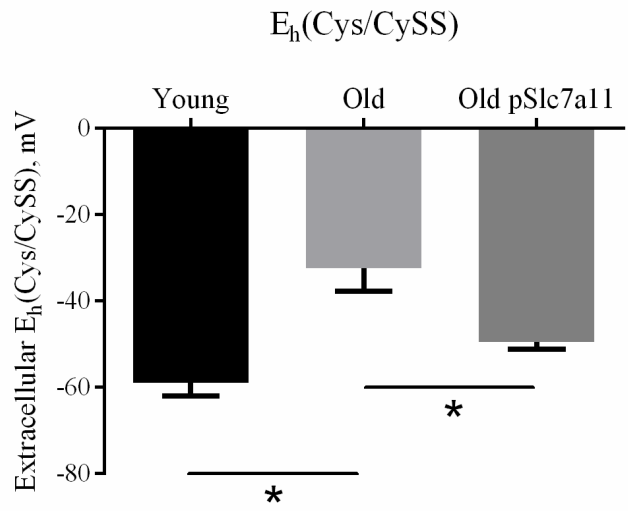

(b)

Figure 3.1 Overexpression of Slc7a11 restored age-dependent oxidation of extracellular $E_{h}$ (Cys/CySS) in old fibroblasts. (a) Slc7a11 mRNA expression and (b) extracellular Eh(Cys/CySS) were measured as described in materials and methods. Primary lung fibroblasts from young and old mice were isolated and old fibroblasts were transfected with Slc7a11-overexpressing plasmid (pSIc7a11). Fibroblasts were plated, and $24 \mathrm{~h}$ later the media were changed to $0 \mathrm{mV}$ redox media for $4 \mathrm{~h}$. Data are expressed as mean \pm standard deviation of 3 independent replicates. ${ }^{*}$ - Indicates $p<0.05$. 
3.3.2 Identification of intracellular protein cysteines whose redox states changed with aging but were restored via Slc7a11 overexpression

After collecting the media for measuring extracellular $\mathrm{E}_{h}$ (Cys/CySS), the cells were processed for intracellular cysteine redox proteomic analysis. Three different iodoTMT labels were used to label biologically reduced cysteine residues of proteins in young, old, and old Slc7a11-overexpressing fibroblasts (Figure 3.2). Next, TCEP was used to reduce reversibly oxidized cysteines, including disulfides, sulfenic acids, S-glutathionylation and S-nitrosylation. Then, 3 different tags were used to label those originally oxidized cysteines. Following digestion and enrichment via iodoTMT-affinity resin, peptides were analyzed via LC-MS/MS. A representative MS/MS spectrum is shown to demonstrate identification of peptides and quantification of oxidized and reduced forms from reporter ions (Figure 3.3). 


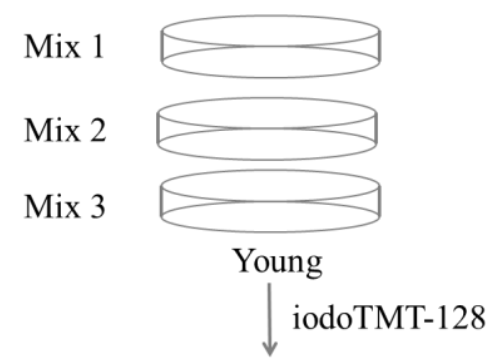

Reduced Cys-128

TCEP $\downarrow$ iodoTMT-129

Reduced Cys-128

Oxidized Cys-129
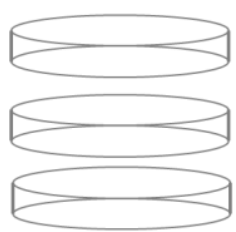

Old

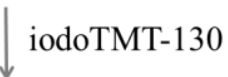

Reduced Cys-130

TCEP $\downarrow$ iodoTMT-131

Reduced Cys-130

Oxidized Cys-131

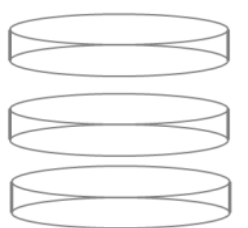

Old Slc7a11 overexpression iodoTMT-126

Reduced Cys-126

TCEP $\downarrow$ iodoTMT-127

Reduced Cys-126 Oxidized Cys-127

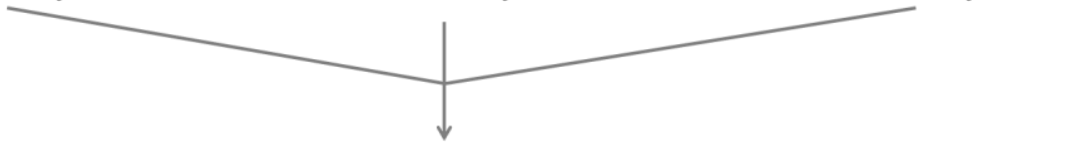

Digestion

Affinity Purification

Multiplexed LC-MS/MS

Figure 3.2 Schematic flow chart of labeling redox-sensitive cysteines in peptides from young, old and old Slc7a11-overexpressing primary mouse lung fibroblasts using iodoTMT reagents. Detailed procedure was described in the main text. 


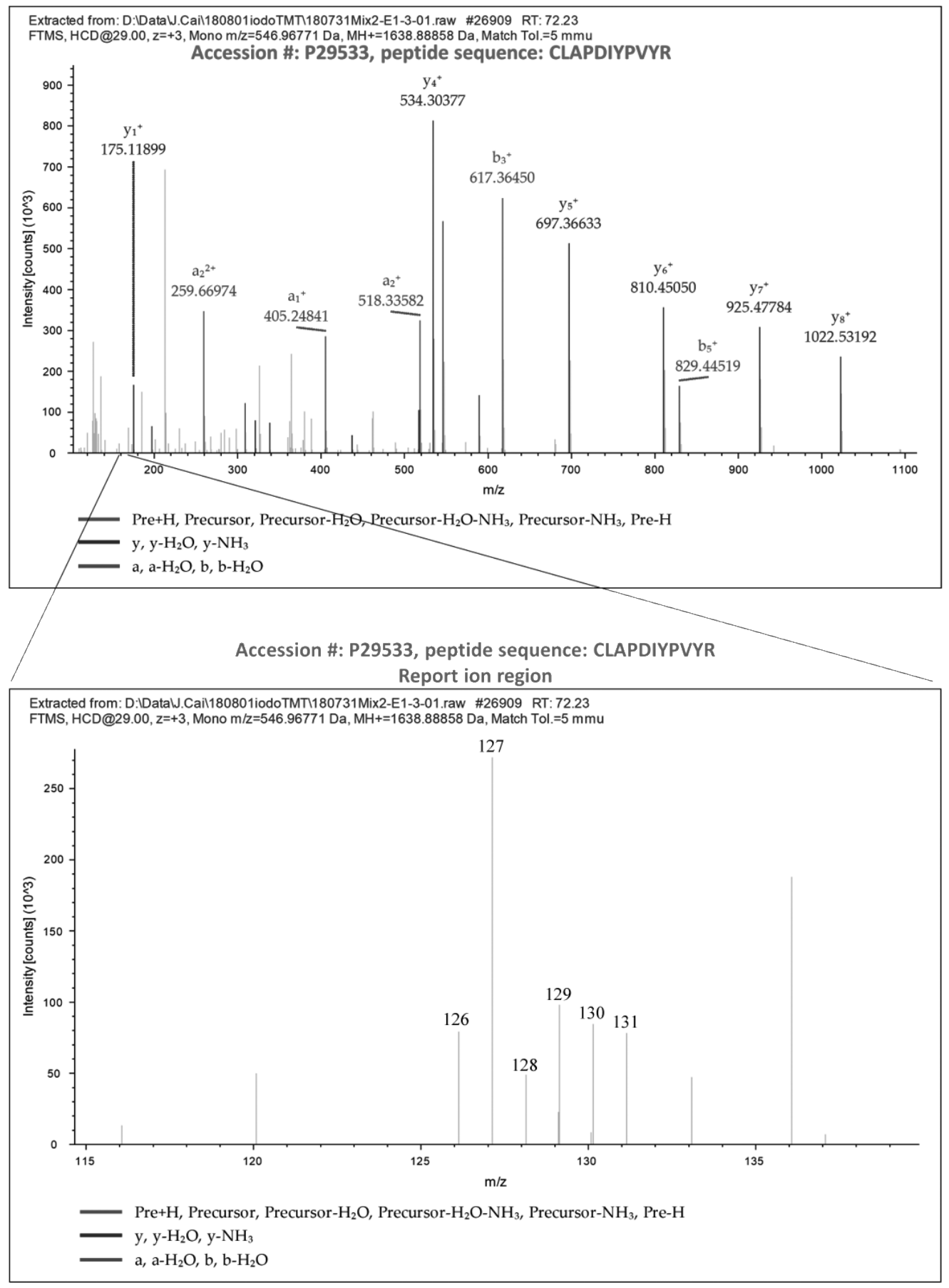

Figure 3.3 Representative mass spectrum for determining Cys redox state. Peptides in the mixed samples were ionized and separated by LC-MS/MS. Specific Cys-containing peptides were selected after the first mass spectrometry based on the mass to charge ratio. Those peptides were further fragmented to smaller ions and detected by the second mass spectrometry as 
shown in the upper panel. The region containing peaks of the reporter ions of the six iodoTMT labels $(\mathrm{m} / \mathrm{z} 126-131)$ is shown in the lower panel. The peak intensity ratios of $129 / 128,131 / 130$ or $127 / 126$ were defined as redox states for young, old or old Slc7a11-overexpressing primary mouse lung fibroblasts, respectively. 
Over one thousand peptides were identified by mass spectrometry. To facilitate quantitative comparisons, the redox state of a specific cysteine residue was represented by the ratio of its oxidized portion to its reduced portion (redox state of cysteine $=$ oxidized portion $/$ reduced portion). To determine how the redox states of individual cysteines differed between old and young cells, a volcano plot representing statistical significance on the $y$-axis and fold-change of cysteine redox states on the $x$-axis was produced (Figure 3.4.a). For each cysteine residue, change of redox state with aging was represented by foldchange (FC) of redox state in old cells relative to young cells (Old vs. Young FC $=$ redox state of cysteine in old cells / redox state of cysteine in young cells) (Figure 3.4.a). Among all cysteines with detectable redox states, 12.6\% (162/1282) peptides contained differentially oxidized cysteines with aging (above the dashed line representing $p$-value cutoff of 0.05 ) (Figure 3.4.a). Of those, $69 \%(112 / 162)$ were more oxidized in the old cells than in the young cells (above the dashed line and $\log _{2} \mathrm{FC}(\mathrm{Old} /$ Young) $>0$ ), while $31 \%(50 / 162)$ were more reduced with aging (above the dashed line and $\log _{2} \mathrm{FC}(\mathrm{Old} /$ Young $)<0$ ) (Figure 3.4.a). 


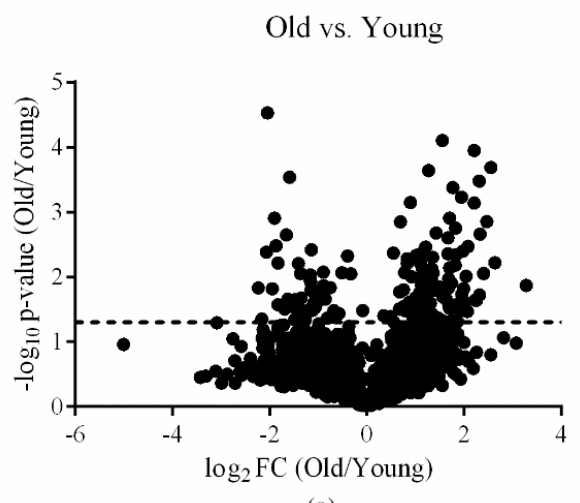

(a)

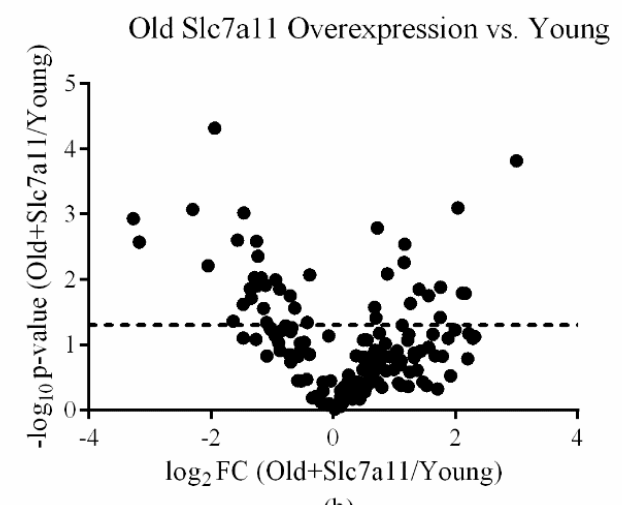

(b)

Figure 3.4 Volcano plot showing peptides with age-dependent Slc7a11reversible Cys redox states. Each dot represented one Cys-containing peptide. (a) Proteins were differentially oxidized between old and young fibroblasts. The peptides above the dashed line were considered to contain cysteines whose redox states changed in response to aging (age-dependent Cys redox state). Those peptides belonged to 151 proteins. (b) Cys redox states were reversed by Slc7a11 overexpression. The proteins containing age-dependent Cys redox states were selected and plotted. Proteins below the dashed line were considered to contain Slc7a11-reversible Cys redox states. Altogether, 104 proteins contained 116 cysteines whose redox states were reversed by SIc7a11 overexpression. Three replicates of young, old and old Slc7a11-overexpressing fibroblasts were used to calculate the $p$-values. 
The 162 peptides whose redox states changed with aging belonged to 151 proteins. These 151 proteins were selected to further explore whether their redox states were reversible via Slc7a11 overexpression. Reversibility of agedependent changes of redox state was assessed by comparing the redox state in old Slc7a11-overexpressing cells relative to young cells (Old Slc7a11 Overexpression vs. Young FC $=$ redox state of cysteine in old Slc7a11overexpressing cells / redox state of cysteine in young cells) (Figure 3.4.b). Noticeably, $71.6 \%(116 / 162)$ of age-dependent changes of cysteine redox states were reversible via Slc7a11 overexpression (below the dashed line) (Figure 3.4.b). The majority of Slc7a11-dependent redox-sensitive cysteines (93 of 116 , or $80 \%$ ) were more oxidized in old cells, but a substantial number (23 of 116 , or $20 \%$ ) were more reduced in old cells. The 116 Slc7a11-dependent cysteine-containing peptides belonged to 104 distinct proteins, with one protein containing both oxidized and reduced cysteines. Those 104 proteins constituted the majority (104 of 151 , or $69 \%$ ) of the age-dependent redoxmodified proteins.

The above observations indicated that decreased expression of Slc7a11 with aging might significantly contribute to the age-dependent global changes observed in the redox states of the intracellular cysteine proteome in primary mouse lung fibroblasts. These 104 proteins with Slc7a11-dependent redoxsensitive cysteines were focused on to further explore their potential biological functions using bioinformatics analyses. 
3.3.3 Ingenuity Pathway Analysis (IPA), Gene Ontology (GO) and pathway enrichment analyses and protein-protein interaction (PPI) network analysis of proteins with Slc7a11-reversed redox-sensitive cysteines

IPA showed that the most significantly enriched pathways related to Slc7a11-dependent redox-sensitive proteins were eukaryotic translation initiation factor 2 (EIF2) signaling, actin cytoskeleton and integrin-linked kinase (ILK) signaling, and protein ubiquitination pathway, which represented protein synthesis, cellular structure and communication, and protein ubiquitinproteasome-mediated degradation, respectively (Table 3.1). Three actinassociated cytoskeleton proteins, TLN1, FLNB and PPP2CA, contained multiple redox-sensitive cysteines, indicating that actin dynamics and communication between cell membrane and intracellular cytoskeleton were prone to age-related oxidative stress and, importantly, that Slc7a11 overexpression restored such redox effects of aging (Table 3.1). Interestingly, not all cysteine residues became more oxidized with aging. On the contrary, some became more reduced (Table 3.1), indicating that although aging promoted overall oxidation of the redox environment, the redox states of distinct components of the intracellular cysteine proteome were differentially regulated instead of simply being oxidized. Slc7a11 overexpression not only reduced agedependent oxidation of proteins, but also oxidized age-dependent reduction of certain proteins, suggesting that Slc7a11 influences the cysteine proteome indirectly via restoring the global intracellular redox environment rather than 
interacting directly with individual redox-sensitive proteins.

$\mathrm{GO}$ is a collection of terms describing gene functions and the relationship between the terms. It consists of 3 aspects: biological process, molecular function and cellular component. Consistent with IPA, GO annotation for biological process revealed that Slc7a11-dependent redox-sensitive proteins were enriched in cell adhesion, initiation and regulation of protein translation, organization of actin cytoskeleton and proteolysis (Table 3.2). Other significant enriched terms related to biological process involved RNA processing, cellular response to stimuli (e.g. virus) and oxidation-reduction processes (Table 3.2). Furthermore, Slc7a11 affected the redox states of proteins with molecular function related to the binding of poly(A) RNA, cellular adhesion-related cadherin, actin, ATP or other proteins (Table 3.3). Related to the GO cellular component, enriched items included extracellular exosome, cell-cell adherens junctions and cytoplasm (Table 3.4). Finally, KEGG pathways were mainly enriched in carbon metabolism and amino acids biosynthesis, ribosome and proteasome, tight and adherens junctions, and focal adhesion (Table 3.5). 
Table 3.1. Ingenuity Pathway Analysis (IPA) indicated proteins whose redox states were restored by Slc7a11 overexpression were enriched in pathways of protein turnover and cytoskeleton signaling. The top 3 most significant enriched pathways were protein synthesis pathway, protein ubiquitin-proteasomemediated degradation pathway and actin cytoskeleton signaling / integrin-linked kinase (ILK) signaling. Some proteins contained more than 1 redox-sensitive Cys residue. Some Cys residues became more oxidized with aging while others became more reduced. 


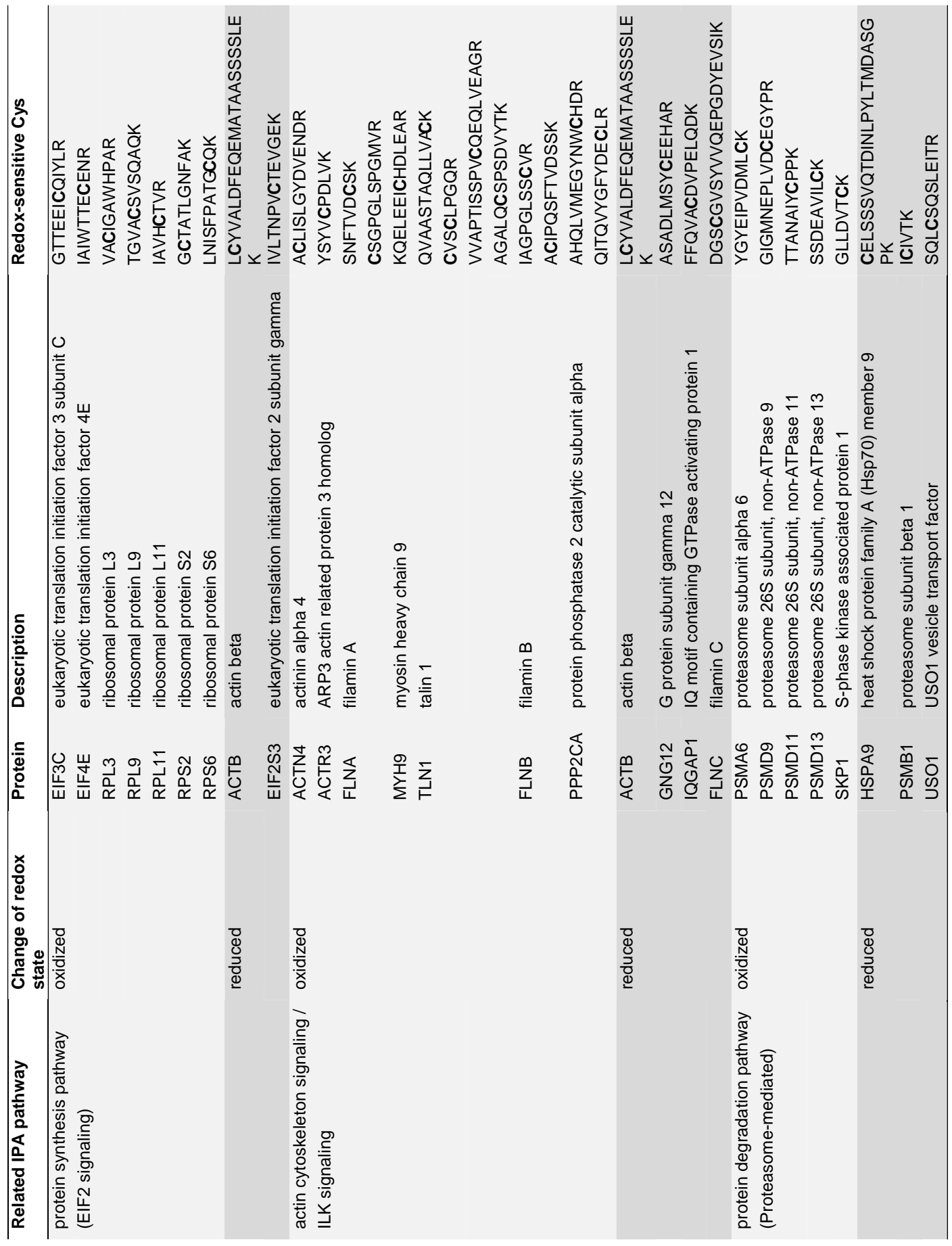


Table 3.2 GO-based biological process analysis of proteins whose redox states were rescued by Slc7a11 overexpression in old fibroblasts. Fisher's exact test p-values for all the items shown were lower than 0.05 .

\begin{tabular}{llcc}
\hline Term & Count & $\boldsymbol{p}$-value & -log10 ( $\boldsymbol{p}$-value) \\
\hline cell-cell adhesion & 11 & 0.000 & 7.197 \\
translation & 11 & 0.000 & 4.274 \\
RNA splicing & 8 & 0.000 & 3.526 \\
tricarboxylic acid cycle & 4 & 0.000 & 3.319 \\
platelet aggregation & 4 & 0.001 & 2.971 \\
mRNA processing & 8 & 0.002 & 2.786 \\
RNA secondary structure unwinding & 4 & 0.002 & 2.757 \\
translational initiation & 4 & 0.003 & 2.529 \\
cellular response to peptide hormone stimulus & 3 & 0.007 & 2.147 \\
formation of translation preinitiation complex & 3 & 0.008 & 2.112 \\
establishment or maintenance of cell polarity & 3 & 0.009 & 2.047 \\
response to virus & 4 & 0.010 & 1.994 \\
pentose-phosphate shunt, non-oxidative branch & 2 & 0.026 & 1.581 \\
oxidation-reduction process & 9 & 0.027 & 1.564 \\
actin cytoskeleton reorganization & 3 & 0.031 & 1.507 \\
isocitrate metabolic process & 2 & 0.031 & 1.503 \\
proteolysis involved in cellular protein catabolic process & 3 & 0.037 & 1.433 \\
positive regulation of translation & 3 & 0.037 & 1.433 \\
neuron projection development & 4 & 0.038 & 1.422 \\
actin cytoskeleton organization & 4 & 0.040 & 1.399 \\
\hline
\end{tabular}


Table 3.3 GO-based molecular function analysis of proteins whose redox states were rescued by Slc7a11 overexpression in old fibroblasts. Fisher's exact test p-values for all the items shown were lower than 0.05 .

\begin{tabular}{llcc}
\hline Term & Count & $\boldsymbol{p}$-value & -log10 ( $\boldsymbol{p}$-value $)$ \\
\hline poly(A) RNA binding & 39 & 0.000 & 20.191 \\
cadherin binding involved in cell-cell adhesion & 20 & 0.000 & 15.060 \\
enzyme binding & 13 & 0.000 & 5.841 \\
actin binding & 12 & 0.000 & 5.558 \\
actin filament binding & 8 & 0.000 & 5.054 \\
RNA binding & 16 & 0.000 & 4.598 \\
protein complex binding & 11 & 0.000 & 4.521 \\
protein binding & 42 & 0.000 & 4.517 \\
nucleotide binding & 25 & 0.000 & 3.935 \\
protein domain specific binding & 9 & 0.000 & 3.682 \\
GTP binding & 9 & 0.001 & 2.878 \\
ATP binding & 19 & 0.001 & 2.826 \\
protein kinase binding & 9 & 0.003 & 2.540 \\
ATP-dependent RNA helicase activity & 4 & 0.005 & 2.284 \\
GTPase activity & 6 & 0.006 & 2.206 \\
oxidoreductase activity & 10 & 0.006 & 2.191 \\
ADP binding & 3 & 0.017 & 1.768 \\
Rac GTPase binding & 3 & 0.022 & 1.662 \\
endopeptidase activity & 4 & 0.025 & 1.610 \\
nucleoside binding & 2 & 0.027 & 1.561 \\
\hline
\end{tabular}


Table 3.4 GO-based cellular component analysis of proteins whose redox states were rescued by Slc7a11 overexpression in old fibroblasts. Fisher's exact test $p$-values for all the items shown were lower than 0.05 .

\begin{tabular}{llcc}
\hline Term & Count & $\boldsymbol{p}$-value & -log10 $(\boldsymbol{p}$-value $)$ \\
\hline extracellular exosome & 57 & 0.000 & 23.688 \\
cell-cell adherens junction & 20 & 0.000 & 14.949 \\
cytoplasm & 72 & 0.000 & 14.704 \\
focal adhesion & 16 & 0.000 & 9.092 \\
myelin sheath & 12 & 0.000 & 8.558 \\
extracellular matrix & 13 & 0.000 & 7.639 \\
cytosol & 28 & 0.000 & 7.085 \\
intracellular ribonucleoprotein complex & 12 & 0.000 & 6.291 \\
spliceosomal complex & 8 & 0.000 & 5.304 \\
actin cytoskeleton & 9 & 0.000 & 5.162 \\
cytoplasmic ribonucleoprotein granule & 5 & 0.000 & 4.983 \\
cortical cytoskeleton & 5 & 0.000 & 4.801 \\
stress fiber & 6 & 0.000 & 4.700 \\
cytoskeleton & 18 & 0.000 & 4.543 \\
perinuclear region of cytoplasm & 14 & 0.000 & 4.457 \\
protein complex & 13 & 0.000 & 4.216 \\
nucleus & 49 & 0.000 & 4.123 \\
extracellular vesicle & 5 & 0.000 & 4.002 \\
catalytic step 2 spliceosome & 6 & 0.000 & 3.947 \\
proteasome complex & 5 & 0.000 & 3.498 \\
\hline
\end{tabular}


Table 3.5 KEGG pathway analysis of proteins whose redox states were rescued by Slc7a11 overexpression in old fibroblasts. Fisher's exact test $p$-values for all the items shown were lower than 0.05 .

\begin{tabular}{llcc}
\hline Term & Count & $\boldsymbol{p}$-value & -log10 $(\boldsymbol{p}$-value $)$ \\
\hline Carbon metabolism & 8 & 0.000 & 4.183 \\
Spliceosome & 7 & 0.001 & 2.972 \\
Tight junction & 7 & 0.001 & 2.873 \\
Citrate cycle (TCA cycle) & 4 & 0.003 & 2.569 \\
Salmonella infection & 5 & 0.005 & 2.317 \\
Proteasome & 4 & 0.007 & 2.147 \\
Biosynthesis of antibiotics & 7 & 0.011 & 1.955 \\
Adherens junction & 4 & 0.025 & 1.597 \\
Biosynthesis of amino acids & 4 & 0.031 & 1.507 \\
Proteoglycans in cancer & 6 & 0.033 & 1.484 \\
Focal adhesion & 6 & 0.035 & 1.453 \\
Ribosome & 5 & 0.039 & 1.414 \\
\hline
\end{tabular}


PPI network demonstrates the interactions between proteins in a straightforward and visualized way. Proteins with functional connections are clustered. Three clusters were identified from the PPI network (Figure 3.5). The upper left cluster was associated with cytoskeleton and cellular interaction, including actin signaling and integrin-linked kinase (ILK) signaling (Figure 3.5). The upper right cluster was involved in protein translation pathways, including EIF2 signaling (Figure 3.5). The lower cluster was related to protein ubiquitination and proteasome-mediated degradation (Figure 3.5). Those data were consistent with the IPA results and suggested that, by reversing the intracellular redox environment, Slc7a11 was able to block the age-dependent changes of redox states of protein cysteines in pathways of cytoskeleton dynamics and protein turnover. 


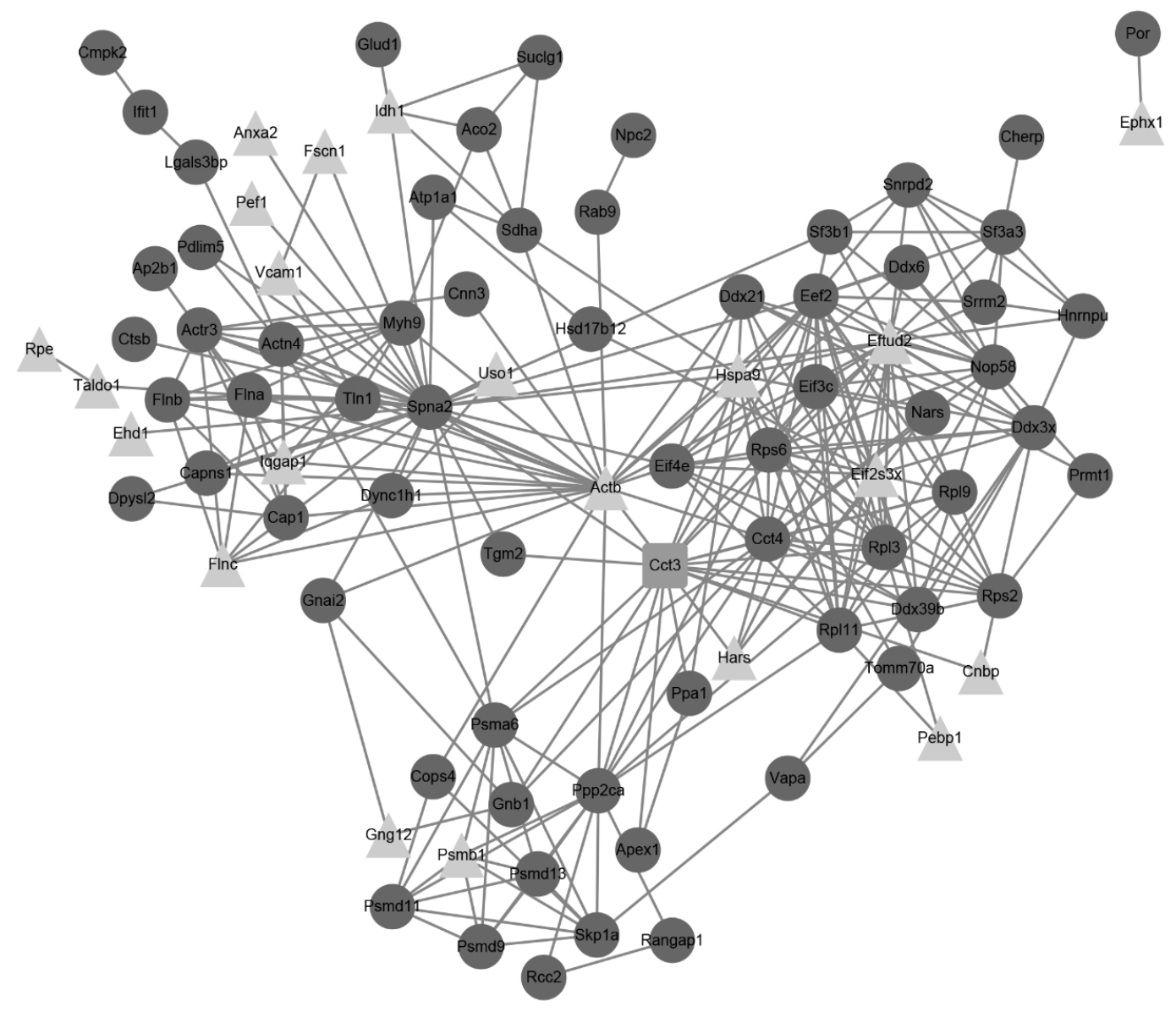

Figure 3.5 Protein-protein interaction (PPI) network showed proteins with agedependent Slc7a11-responsive cysteines were clustered in IPA-predicted pathways. The upper left cluster was associated with cytoskeleton and cellular interaction, including actin signaling and integrin-linked kinase (ILK) signaling. The upper right cluster was associated with protein translation, including eukaryotic translation initiation factor 2 (EIF2) signaling. The lower cluster was related to protein ubiquitination and degradation. Proteins that contained agedependent oxidized, reduced, or both oxidized and reduced cysteines were shown in round, triangular or round rectangular boxes, respectively. 


\subsection{Discussion}

The current study confirms the role of Slc7a11 in mediating age-dependent oxidization of extracellular $E_{h}$ (Cys/CySS) and extends these findings to include the impact of changes in Slc7a11 expression on the redox states of multiple intracellular proteins. Proteins related to the cytoskeleton or involved in protein degradation and protein synthesis are differentially oxidized in old murine lung fibroblasts as a result of lower Slc7a11 expression. Slc7a11 overexpression not only reduces proteins that become oxidized with aging, but also oxidizes certain proteins that are reduced with aging. Thus, targeting Slc7a11 for redox regulation might be a better approach than simply supplementing with antioxidants to re-establish redox homeostasis in aging and diseases.

The redox states of particular cysteines associated with actin cytoskeleton signaling and ILK signaling are age-dependent but can be restored with Slc7a11 overexpression. Integrins mediate cell-cell and cell-matrix interactions, thereby linking extracellular matrices with the intracellular cytoskeleton. Talin and alpha-actinin, which are components of focal adhesion complexes (219, 220), are shown in Chapter 3 to contain cysteines whose redox states are oxidized with aging. These changes might impact integrin-mediated signaling or mechanotransduction through alterations in cell stiffness, a process considered critical for promoting aberrant lung remodeling after injury and fibrosis $(221,222)$. Others have shown that cytoskeletal proteins are oxidized with aging. Protein carbonylation is an irreversible oxidative modification that is 
increased in the brains of old senescence-accelerated-prone 8 (SAMP8) mice compared to the brains of young mice (223). In particular, collapsin response mediator protein-2 (CRMP-2), which is involved in cytoskeletal remodeling, microtubule assembly and cell migration, and alpha-spectrin, which interacts with actin to form a scaffold to maintain cytoskeletal stability and flexibility, are more oxidized (224). Thus, the actin cytoskeleton system might be a major target of age-dependent redox modification. S-glutathionylation is a form of reversible oxidation of cysteines. Actin contains cysteines with different degrees of S-glutathionylation that are flexibly deglutathionylated via glutaredoxin in response to stimuli (225). Glutaredoxin-mediated changes of cysteine Sglutathionylation may regulate actin polymerization (226). These findings are considered important as the actin cytoskeleton is believed to play critical roles in fibroblast differentiation, myofibroblast contraction, focal adhesion complex formation, extracellular matrix remodeling, mechanical to biochemical signal transduction, and gene transcription, which are all important biological processes proposed to be involved in tissue fibrogenesis and maladaptive wound healing (227). In human skin fibroblasts, disassembly of actin cytoskeleton results in disruption of the TGF- $\beta$ signaling pathway with subsequent collagen production, thus promoting skin aging (228). These findings further support the idea that the function of the actin cytoskeleton system is redox-sensitive, and its disruption might be associated with agingrelated degeneration and disease development. 
Loss of proteostasis is a hallmark of aging (27). Proteostasis (from the terms protein and homeostasis) refers to the balance among the processes of protein synthesis, folding, trafficking and degradation. Redox modulation has been shown to affect protein folding and trafficking $(229,230)$. Disruption of proteostasis can result in deposition of protein aggregates and be harmful for cell survival $(231,232)$. Protein degradation by the proteasome changes with aging and is regulated by redox modulation, especially thiol oxidation $(233,234)$. Here, three age-oxidized cysteines in proteasome $26 \mathrm{~S}$ subunit and one agereduced cysteine in heat shock protein 70 (HSP70) were identified, providing further evidence for the idea that the $26 \mathrm{~S}$ proteasome is vulnerable to oxidative stress, and that its chaperone HSP70 responds to redox regulation $(235,236)$. The implications of age-dependent changes of protein redox states in the promotion of aging-associated diseases require further exploration. However, studies in Chapter 3 suggest that they are important and are in line with other observations. For instance, in the hippocampi of patients with Alzheimer disease (AD), peptidyl-prolyl cis-trans isomerase 1 (Pin 1), which catalyzes isomerization of neuronal cytoskeleton protein tau, becomes more oxidized (237). Ubiquitin carboxyl terminal hydrolase L-1 (UCHL-1) is involved in proteasome-mediated protein degradation, and its oxidation in AD disrupts the ubiquitination/de-ubiquitination balance and results in accumulation of dysfunctional proteins (237). Furthermore, proteins in the cell structural and proteasomal pathways are oxidatively modified in Parkinson disease (238). 
Others have identified cysteine redox modifications that affect proteostasis. For example, S-glutathionylation of multiple cysteine residues results in increased accessibility of proteasomal active center and increased proteolytic activity (239). In this chapter, several other novel reversible redox-sensitive cysteines that might also influence proteasomal activity were identified. Whether proteosomal activity is increased or decreased depends on both the location and specific form of the redox modification being evaluated, thereby requiring experimental exploration in the future. Nevertheless, observations in Chapter 3 suggest that both cytoskeleton and proteasomal pathways are susceptible to age-related oxidative stress, and that redox modulations of cysteines might be an important post-translational mechanism responsible for age-dependent change of protein functions in those pathways.

Protein synthesis was also found to be disrupted with aging and modified by Slc7a11-associated change of the redox environment. Compared to relatively well studied age-dependent protein degradation, how protein synthesis is altered with aging remains to be explored. My study found that aging was associated with changes of redox states of cysteines in EIF2 signaling, including EIF4E, RPS6 and RPL9. These three proteins regulate protein synthesis in aging (240). The concentration and activity of eukaryotic translation initiation factors (elFs) and the abundance of ribosomes are decreased with aging, resulting in an overall decrease in protein synthesis (241). These and the identification of several other age-dependent cysteines in other 
parts of elFs and ribosomes suggest that cysteine redox modifications mediate age-dependent defects detected in the protein synthesis machinery. The function of elF2, for instance, is to load methionyl-tRNA to $40 \mathrm{~S}$ ribosomal subunit, which is the first step of $43 S$ preinitiation complex assembly (242). The function of elF4E is to bind with the 5' cap of mRNAs and mediate recruitment and attachment of $43 S$ preinitiation complex (243). Those two steps determine the rate of protein synthesis. Phosphorylation of those initiation factors and their regulatory factors have been shown to control protein synthesis $(244,245)$. Similar to phosphorylation, redox modifications might represent another posttranslational mechanism capable of affecting the efficacy of protein synthesis. This idea is further supported by a recent study that revealed several $\mathrm{H}_{2} \mathrm{O}_{2}$ sensitive thiols in proteins involved in the general translation machinery, and the subsequent attenuation of protein synthesis via such redox modulation (246). More studies are needed to further elucidate this redox control of protein synthesis pathways.

Perhaps the most important finding of Chapter 3 is that Slc7a11 overexpression may restore the protein redox microenvironments. Accordingly, one could predict that alterations in Slc7a11 expression or activity would lead to diseased states. Consistent with this idea, others reported that increase of Slc7a11 was part of the signatures of senescence inflammatory responses in intestinal epithelial cells (247). In peripheral white blood cells from schizophrenia patients, Slc7a11 expression was lower compared to those from 
healthy donors (248). In tissues of non-small cell lung cancer, Slc7a11 expression was higher (249). In glioma cells, the C-terminus of EGFR directly interacts with the central part of Slc7a11 and stabilizes Slc7a11 cell surface expression (250), thereby suggesting a role in oncogenic signaling. CD44 variant directly interacts with Slc7a11 in pulmonary artery endothelial cells suggesting a role in vascular remodeling (251). Those interactions further complicate the potential biological roles of Slc7a11. It is unclear whether and how Slc7a11 expression changes in different cell types and whether such change affect the progression of aging or disease development differently. Although the upstream or downstream pathways of Slc7a11 might be different, the universal underlying mechanism is usually associated with Slc7a11-related change of redox environment, suggesting redox modulation might play a central role in controlling multiple biological processes and signaling pathways.

SIc7a11 provides cells with Cys to support GSH synthesis. Therefore, one of the functions of Slc7a11 activity may be to support reduction of oxidized proteins by providing an essential co-factor for glutaredoxins (94). Glutaredoxins preferentially reduce glutathionylated proteins, whereas thioredoxins more efficiently reduce protein disulfides and sulfenic acids and are involved in de-nitrosylation of cysteines $(87,252-255)$. The link between Slc7a11 activity and thioredoxin-dependent protein reduction is not as direct as it is for glutathione-dependent processes, but an increase in GSH synthesis may decrease the overall burden on the thioredoxin system. The results in 
Chapter 3 showed that while most cysteines became more oxidized in lung fibroblasts from aged mice, some became more reduced, and Slc7a11 overexpression reversed redox states of both oxidized and reduced cysteines, supporting the idea that Slc7a11 manipulation targets intracellular redox signaling pathways rather than simply decreases overall oxidative stress. This might provide novel insight for combatting oxidative stress associated with aging and age-related aberrant wound healing.

In conclusion, aging is associated with disruption of the intracellular redox environment represented by changes in the redox states of cysteines in fundamental pathways including protein turnover and cytoskeleton organization. Slc7a11 is a critical factor not only for regulating the extracellular redox environment, but also for controlling the intracellular redox environment. Targeting Slc7a11 can reverse age-dependent effects of oxidative stress on intracellular signaling pathways and lead to novel approaches to fighting against oxidative stress in age-related diseases. 
CHAPTER IV

\author{
DECREASED SLC7A11 IS ASSOCIATED WITH OXIDATION OF \\ EXTRACELLULAR CYSTEINE/CYSTINE REDOX STATE (EH(CYS/CYSS)) \\ IN HUMAN LUNG FIBROBLASTS: A POTENTIAL SUSCEPTIBILITY STATE \\ FOR IDIOPATHIC PULMONARY FIBROSIS
}

\title{
4.1 Introduction
}

Idiopathic pulmonary fibrosis (IPF) is the most common form of interstitial lung disease (256). It is characterized by irreversible destruction of alveoli, accumulation of aberrant matrix proteins in the lung interstitium, and remodeling of lung tissue resulting in scarring (257). The main clinical symptoms are dry cough and worsening shortness of breath, and the physical signs include bibasilar pulmonary crackles and finger clubbing (3). Oxygen supplementation is often needed as pulmonary function declines. The burden of IPF is not negligible. Over 180,000 Americans are currently affected by IPF, and the incidence as well as prevalence is still increasing worldwide $(6,258)$. IPF is insidious and progressive, usually diagnosed at later stages because of its chronic and non-specific nature. Even though the progression of IPF can be decelerated by pirfenidone or nintedanib, it's still lethal because no intervention 
has been proved to halt or reverse the fibrosis $(18,19)$. Furthermore, IPF mainly affects people from 55 to 75 years of age. The median age at diagnosis is 66 and the median survival time is 3 to 5 years $(12,259)$. As the world population is aging, it is imperative to explore new diagnostic approaches for detecting IPF during its initial stage or new treatment options capable of reversing the fibrotic process. Thorough understanding of the mechanisms underlying IPF on the cellular and molecular levels is fundamental for developing innovative diagnostic biomarkers and effective treatments.

Lung fibroblasts from IPF patients are different than those from non-IPF donors. Fibroblasts are the effector cells that produce excessive extracellular matrix proteins and manifest a fibrotic phenotype in IPF. IPF fibroblasts show decreased proliferation, decreased telomere length, enlarged morphology, increased resistance to apoptosis, increased expression of senescent markers ( $\beta$ - galactosidase, p16, p21, p53), and increased cytokine production (interleukin-6, interleukin-1 $\beta$, fibroblast growth factor 2 ) belonging to the senescence-associated secretory phenotype (SASP), all of which are characteristics of cellular senescence $(29,260)$. Furthermore, media from senescent fibroblasts stimulate the expression of $\alpha$-smooth muscle, collagen and fibronectin in non-senescent fibroblasts, indicating that senescent fibroblasts are pro-fibrotic and fibroblast senescence might play a key role in mediating IPF (261).

Redox homeostasis is disrupted in IPF fibroblasts. For example, 
mitochondrial dysfunction has been documented in IPF fibroblasts, including over-production of reactive oxygen species (ROS), up-regulation of genes related with mitochondrial synthesis and electron transport chain, increased mitochondrial DNA content and increased activity of mammalian target of rapamycin complex 1 (mTORC1), a mitochondrial homoeostasis mediator (111). IPF fibroblasts also express increased NADPH (reduced nicotinamide adenine dinucleotide phosphate) oxidase 4 (NOX4) (96). Expression of nuclear factor, erythroid 2 like 2 (NRF2), a major regulator of multiple anti-oxidant genes, is absent in fibroblast foci of IPF lung tissue (96). Both mitochondrial dysfunction and disturbance of the NOX4-NRF2 axis may promote fibroblast senescence and differentiation into myofibroblast and myofibroblast apoptosis resistance resulting in increased susceptibility to lung fibrosis after injury $(96,111)$.

Redox couple together with its redox potential is another type of readily available biomarker of redox environment. Cysteine (Cys) and its oxidized form, cystine (CySS), constitute the major extracellular redox couple. Glutathione/glutathione disulfide (GSH/GSSG) is the major intracellular redox couple. Previous studies show extracellular Cys/CySS redox potential $\left(E_{h}(\right.$ Cys/CySS $\left.)\right)$ is more oxidized for cultures of fibroblasts from old mice compared to young mice (157). Oxidation of extracellular $E_{h}$ (Cys/CySS) promotes pro-fibrotic responses of primary mouse lung fibroblasts, including activation of TGF- $\beta /$ Smad signaling and induction of extracellular matrix proteins, such as fibronectin (156). Plasma En(Cys/CySS) and En(GSH/GSSG) 
are progressively oxidized with aging in humans (137). Considering aging is the biggest risk factor for IPF, oxidation of redox couples might potentially mediate the effects of aging on promoting lung fibrosis. In the bleomycin-induced lung injury murine model, oxidation of plasma $E_{h}($ Cys/CySS) coincides with fibrosis while oxidation of plasma $E_{h}(\mathrm{GSH} / \mathrm{GSSG})$ precedes the appearance of fibrosis (57). Follow-up study in the mouse lung fibroblasts demonstrates that solute carrier family 7 (cationic amino acid transporter, y+ system), member 11 (Slc7a11), the light chain subunit of system $\mathrm{X}_{c}^{-}$importing CySS and exporting glutamate, is the critical molecule for controlling extracellular $E_{h}$ (Cys/CySS) (203). Lower Slc7a11 correlates with more oxidizing extracellular $\mathrm{E}_{h}$ (Cys/CySS) (203). The relationship between Slc7a11 and GSH/GSSH redox couple is less studied. Furthermore, Slc7a11 affects cysteine thiol oxidation status of intracellular proteins involved in pathways of protein turnover and cytoskeleton dynamics. These data suggest targeting Slc7a11 might be a candidate for interfering development of lung fibrosis.

However, there is no study focusing on the role of redox couples and their redox potentials in IPF fibroblast pathology. Whether solute carrier family 7 member 11 (SLC7A11) has similar regulatory effects on redox environments for human lung fibroblasts remains to be explored. The purpose of this study is to characterize extracellular and intracellular redox potentials for lung fibroblasts from IPF patients and non-IPF donors and to examine SLC7A11 expression and its relationship with redox couples in human fibroblasts. 
4.2 Materials and methods

\subsubsection{Reagents}

Reagents used in this paper were all purchased from Sigma-Aldrich (St. Louis, MO) or Corning (Manassas, VA) unless otherwise specified.

4.2.2 Fibroblast cultures from IPF patients and non-IPF donors

Six lung specimens from explants of 6 IPF patients receiving lung transplantation in the University of Louisville Hospital were collected and used for IPF fibroblast isolation. All patients enrolled in this study provided written consent. This study was approved by University of Louisville Hospital Ethics Committee. Fresh tissues were transported immediately to the laboratory for isolating fibroblasts according to procedures described in Chapter $2(176,262)$. Non-IPF fibroblasts were kindly provided by Dr. Halayko. They were isolated from uninvolved periphery of 6 lung cancer patients. Those cancer-adjacent cells were used as controls. These human lung fibroblasts were cultured in Dulbecco's Modified Essential Medium (DMEM) with 10\% fetal bovine serum (FBS) and 1\% antibiotic-antimycotic mixture. Culture dishes were kept in a humidified incubator at $37^{\circ} \mathrm{C}$ with $5 \% \mathrm{CO}_{2}$. Fibroblasts with passage numbers before 10 were used in the experiments. Age and sex data of IPF patients and non-IPF donors are presented in Table 4.1. 
Table 4.1 Age and sex of IPF patients and non-IPF donors

\begin{tabular}{ccc}
\hline Patient number & Age & Sex \\
\hline IPF-1613 & 68 & Male \\
IPF-1705 & 65 & Male \\
IPF-1710 & 67 & Male \\
IPF-1713 & 71 & Male \\
IPF-1714 & 62 & Female \\
IPF-1715 & 64 & Male \\
Non-IPF-004 & 69 & Female \\
Non-IPF-005 & 64 & Male \\
Non-IPF-090 & 56 & Female \\
Non-IPF-103 & 80 & Female \\
Non-IPF-128 & 57 & Female \\
Non-IPF-138 & 55 & Male \\
\hline
\end{tabular}


4.2.3 $0 \mathrm{mV}$ redox media preparation and fibroblast incubation

DMEM without L-methionine, L-cystine and L-glutamine were used as the base media for preparation of $0 \mathrm{mV}$ redox media. $0 \mathrm{mV}$ media contained 0.2 $\mathrm{mM}$ L-methionine, $4 \mathrm{mM}$ L-glutamine, $99.75 \mu \mathrm{M}$ L-cystine and $0.5 \mu \mathrm{M} \mathrm{L}-$ cysteine. All $0 \mathrm{mV}$ redox media were prepared immediately before media change. Fibroblasts were incubated with $0 \mathrm{mV}$ redox media for $24 \mathrm{~h}$.

4.2.4 Derivatization of media and analysis of high-performance liquid chromatography (HPLC)

Conditioned media were combined with the same amount of ice-cold solution composed of $200 \mathrm{mM}$ boric acid, $10 \%(\mathrm{w} / \mathrm{v})$ perchloric acid and $20 \mu \mathrm{M}$ Y-glutamyl glutamate (177). lodoacetic acid and dansyl chloride were used to derivatize the media for HPLC analysis (Waters Corporation, Millford, MA) (134, 178). Calculations of $E_{h}\left(\right.$ Cys/CySS) and $E_{h}(G S H / G S S G)$ are based on the Nernst equation for $\mathrm{pH} 7.4$ : $\mathrm{E}_{\mathrm{h}}(\mathrm{Cys} / \mathrm{CySS})=-250+30^{*} \log \left([\mathrm{CySS}] /[\mathrm{Cys}]^{2}\right)$ and $E_{h}(G S H / G S S G)=-264+30 * \log \left([G S S G] /[G S H]^{2}\right)(203)$.

\subsubsection{Measurement of SLC7A11, COL1A1 and ACTA2 mRNA level}

RNAqueous ${ }^{\circledR}-4$ PCR Kit (Thermo Fisher Scientific, Waltham, MA) was used for isolating RNA from human lung fibroblasts of 5 IPF patients and 5 non-IPF donors without $0 \mathrm{mV}$ redox media incubation. Non-IPF-103 and IPF-1714 were not included due to cell culture issues. SuperScript ${ }^{\mathrm{TM}}$ VILO ${ }^{\mathrm{TM}}$ Master Mix Kit (Thermo Fisher Scientific, Waltham, MA) was used to synthesize cDNA. Real- 
time quantitative PCR (qPCR) was conducted to measure SLC7A11, COL1A1, ACTA2 mRNA and eukaryotic 18S rRNA (18S) expression with TaqMan probes (TaqMan® Gene Expression Assay Hs00921938_m1, Hs00164004_m1, Hs00426835_g1 and Hs99999901_s1; Applied Biosystems), according to the manufacturer's protocol (TaqMan Universal Master Mix II; Applied Biosystems). Step One Plus Real Time PCR System (Applied Biosystems) was used for qPCR. The following cycle parameters were used: $50^{\circ} \mathrm{C}$ for $2 \mathrm{~min}, 95^{\circ} \mathrm{C}$ for $10 \mathrm{~min}$, followed by 40 cycles at $95^{\circ} \mathrm{C}$ for $15 \mathrm{~s}$ and $60^{\circ} \mathrm{C}$ for $1 \mathrm{~min}$. Results were analyzed using Step One Software version 2.3 (Applied Biosystems). The amplification curve was analyzed by the mathematical equation of the second derivative, and the amounts of SLC7A11, COL1A1, ACTA2 mRNA expression were normalized to the housekeeping gene $18 \mathrm{~S}$ rRNA expression. Relative change compared to the average of non-IPF fibroblast samples were calculated for each cell isolate. Finally, relative expression of mRNA was calculated via using the $2^{-\triangle \Delta C T}$ method as in Chapter 2.

\subsubsection{Statistical analysis}

Data were presented as mean \pm standard deviation. Significance was evaluated by unpaired two-tailed $t$-test. Linear regression was used to assess the correlation between redox potential and SLC7A11 expression.

\subsection{Results}

\subsubsection{Extracellular $E_{h}($ Cys/CySS) for human lung fibroblasts}

Primary lung fibroblasts isolated from IPF patients produced more oxidized 
extracellular $E_{h}($ Cys/CySS) than fibroblasts from non-IPF controls (Figure 4.1.A). The average extracellular $\mathrm{Eh}$ (Cys/CySS) was $-37 \mathrm{mV}$ for IPF fibroblasts and $-70 \mathrm{mV}$ for non-IPF fibroblasts (Figure 4.1.A). Because $\mathrm{E}_{h}$ (Cys/CySS) was calculated from concentrations of Cys and CySS. I then looked at their individual concentrations. Data from 6 IPF and 6 non-IPF patients suggested that the average extracellular Cys concentration was $4 \mu \mathrm{M}$ lower for IPF fibroblasts, accounting for this approximately $33 \mathrm{mV}$ oxidation of $\mathrm{E}_{h}$ (Cys/CySS) (Figure 4.1.B). Such oxidation and the decrease in Cys were consistent with the previous mouse study that compared to young mice, extracellular $E_{h}($ Cys/CySS) was more oxidized for fibroblasts from aged mice whose susceptibility to lung fibrosis was also higher $(157,203)$. There was no difference of extracellular CySS concentration between IPF and non-IPF fibroblasts (Figure 4.1.C). 

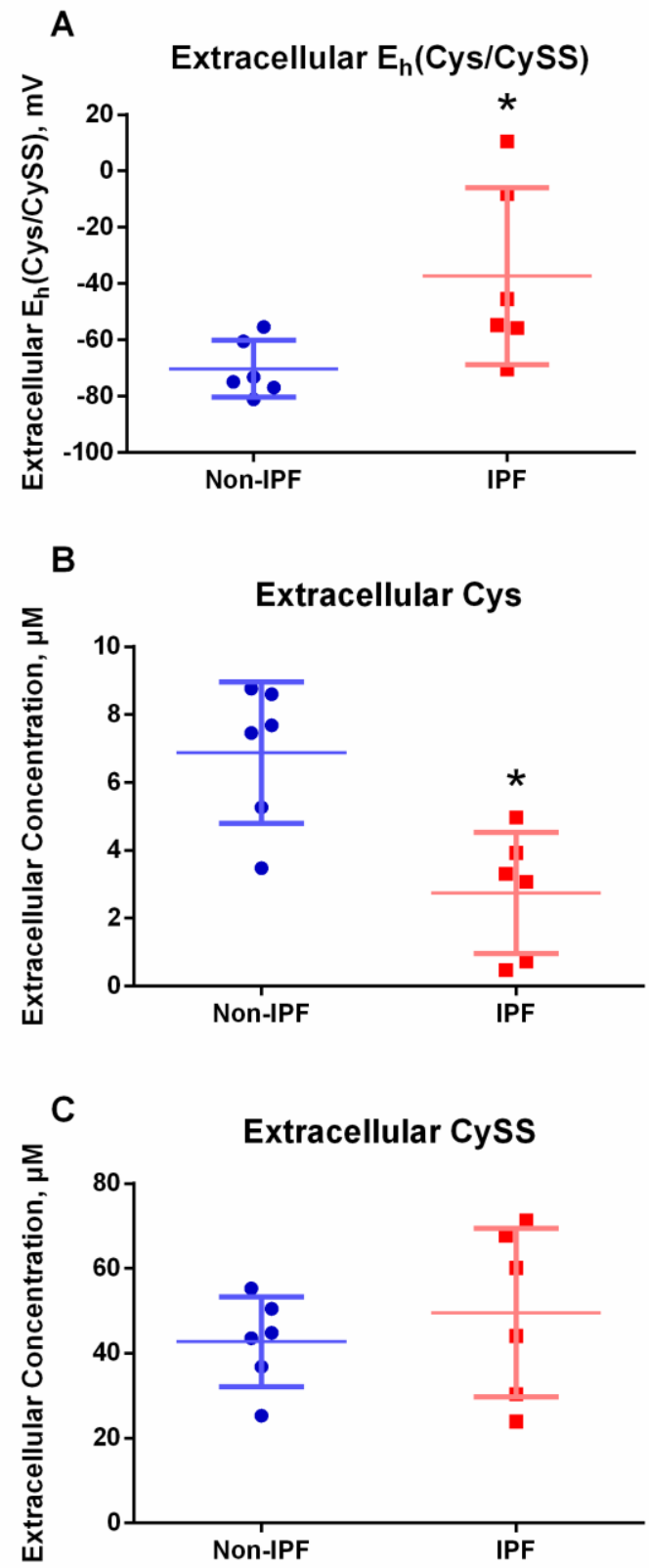

Figure 4.1 Extracellular $\mathrm{E}_{h}$ (Cys/CySS) became more oxidized for lung fibroblasts from IPF patients due to lower extracellular Cys concentration. Lung fibroblasts from uninvolved periphery of lung tissues of 6 lung cancer patients (non-IPF) and 6 IPF patients were isolated and cultured in DMEM containing $10 \%$ fetal bovine serum. When the cultures were $50 \%$ confluent, media were replaced with serum-free media containing $100 \mu \mathrm{M}$ CySS ( $0 \mathrm{mV}$ redox media) for 24 hours. Media were collected to measure concentrations of Cys and CySS. 
Extracellular $E_{h}$ (Cys/CySS) was calculated using Nernst equation according to the following formula: $E_{h}($ Cys $/$ CySS $)=-250+30^{*} \log \left([\right.$ CySS $\left.] /[\text { Cys }]^{2}\right) .(A)$ Extracellular $E_{h}($ Cys/CySS) was more oxidized for IPF fibroblasts. (B) Extracellular Cys concentration was lower for IPF fibroblasts. (C) Extracellular CySS concentration was not altered for IPF fibroblasts. *- Indicates p-value $<$ 0.05 compared to non-IPF fibroblasts. 
4.3.2 Expressions of SLC7A11 and pro-fibrotic genes for human lung fibroblasts Consistent with the hypothesis, SLC7A11 expression was 13-fold lower in IPF fibroblasts, which could account for the oxidation of extracellular $\mathrm{En}_{\mathrm{h}}$ (Cys/CySS) (Figure 4.2). Collagen type I (COL1A1), which is a typical extracellular matrix protein, and actin $\alpha-2$, smooth muscle (ACTA2), which represents differentiation of fibroblasts into the more pro-fibrotic myofibroblasts, were shown to be higher in IPF fibroblasts (Figure 4.3). 


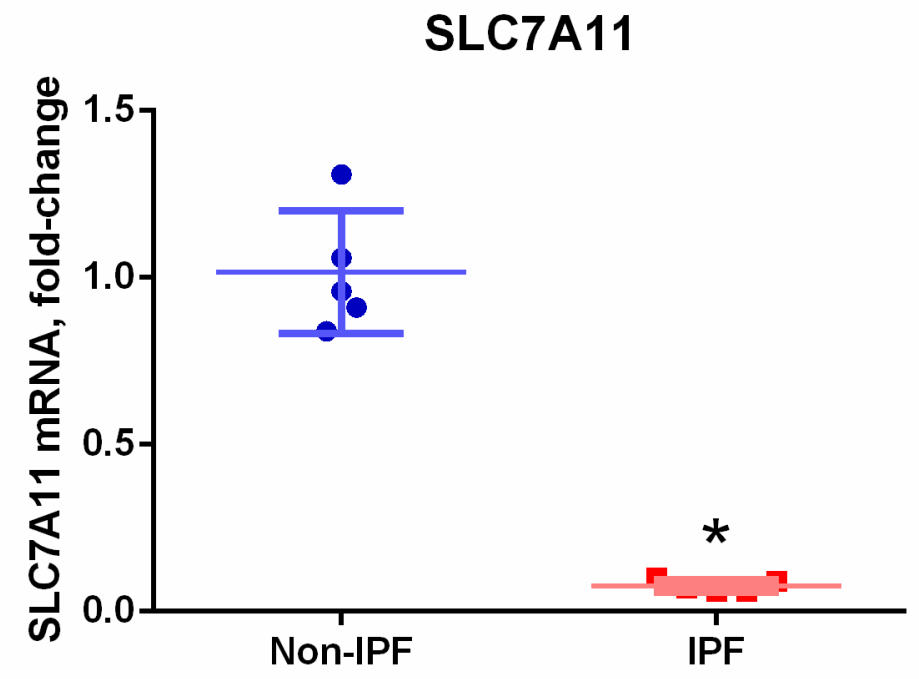

Figure 4.2 SLC7A11 expression was lower for IPF fibroblasts. *- Indicates pvalue $<0.05$ compared to non-IPF fibroblasts. 

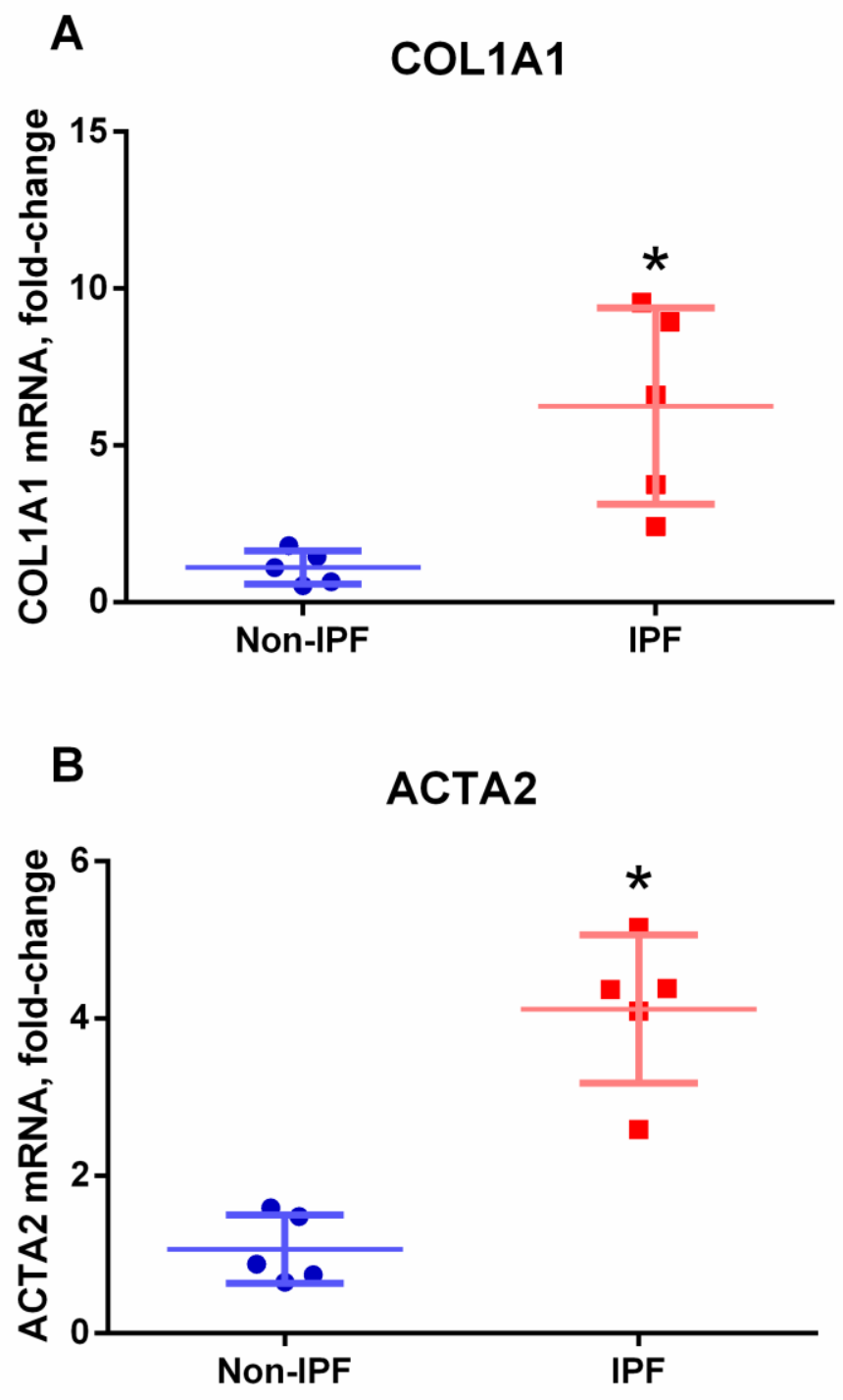

Figure 4.3 Expressions of pro-fibrotic genes were higher for IPF fibroblasts. (A) COL1A1 expression was higher for IPF fibroblasts. (B) ACTA2 expression was higher for IPF fibroblasts. *- Indicates $p$-value $<0.05$ compared to non-IPF fibroblasts. 
4.3.3 Intracellular $E_{h}(\mathrm{GSH} / \mathrm{GSSG})$ for human lung fibroblasts

GSH/GSSG is the major intracellular redox couple. A further step was taken to investigate whether intracellular $\mathrm{E}_{\mathrm{h}}(\mathrm{GSH} / \mathrm{GSSG})$ was different between IPF and non-IPF fibroblasts. In contrast to extracellular $E_{h}$ (Cys/CySS), intracellular $E_{h}(\mathrm{GSH} / \mathrm{GSSG})$ was not more oxidized in IPF fibroblasts (Figure 4.4). 


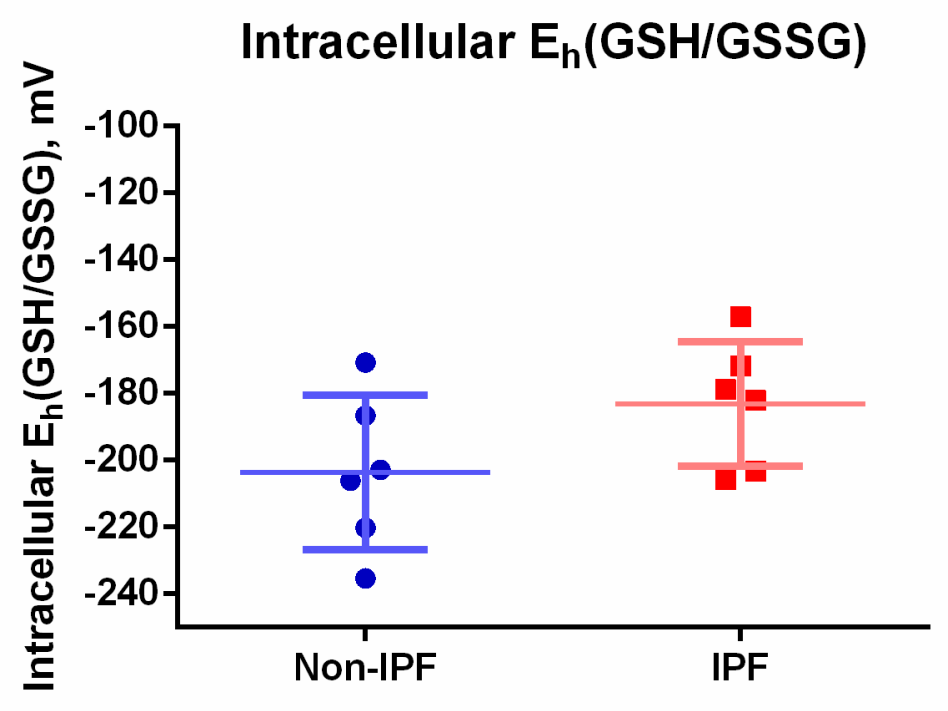

Figure 4.4 Intracellular $\mathrm{E}_{h}(\mathrm{GSH} / \mathrm{GSSG})$ was not more oxidized for IPF fibroblasts. After incubation with $0 \mathrm{mV}$ redox media for 24 hours, cells were collected for measuring intracellular GSH and GSSG concentrations by HPLC. Intracellular $\mathrm{Eh}(\mathrm{GSH} / \mathrm{GSSG})$ was calculated based on Nernst equation using the following formula: $E_{h}(G S H / G S S G)=-264+30^{*} \log \left([G S S G] /[G S H]^{2}\right)$. 


\subsection{Discussion}

The increase of a variety of biomarkers of oxidative stress has been observed in several IPF-derived samples $(34,60)$. Redox couples, Cys/CySS and GSH/GSSG, are among the most commonly used markers to indicate disruptions of redox homeostasis $(75,76,80,81)$. Plasma $E_{h}($ Cys/CySS) is around $-80 \mathrm{mV}$ for healthy elderly people (134). There is about $15 \mathrm{mV}$ oxidation of plasma $E_{h}$ (Cys/CySS) for the age-matched IPF patients according to unpublished data in our group. In the current study, the finding was extended that $E_{h}($ Cys/CySS) became more oxidized not only in human plasma, but also in media of human lung fibroblast cultures. Furthermore, decreased Slc7a11 expression has been shown to be associated with more oxidized extracellular $E_{h}($ Cys/CySS) in mouse lung fibroblasts in Chapter 2. This correlation was also observed in human lung fibroblasts in this Chapter. IPF fibroblasts expressed lower SLC7A11 and their extracellular $E_{h}$ (Cys/CySS) was more oxidized. Previous studies also showed media with oxidized $E_{h}($ Cys/CySS) increased pro-fibrotic gene expression in primary mouse lung fibroblasts (156). The profibrotic effect of oxidation of extracellular $E_{h}$ (Cys/CySS) was confirmed in human fibroblasts in this chapter. Two representative fibrotic genes, COL1A1 and ACTA2, were shown to be upregulated in IPF fibroblasts. In other words, SLC7A11-dependent reduction of extracellular $E_{h}$ (Cys/CySS) in human fibroblasts was associated with lower pro-fibrotic gene expression, decreasing the susceptibility of fibrosis. Those data indicate that SLC7A11 might be a 
potential anti-fibrotic target for intervening redox environments before IPF development and during IPF pathogenesis. Manipulating SLC7A11 might be effective in preventing and treating IPF.

Regulation of intracellular $\mathrm{E}_{\mathrm{h}}$ (GSH/GSSG) was independent of SLC7A11. Though SLC7A11 was lower in IPF fibroblast, intracellular Eh(GSH/GSSG) was not different between IPF and non-IPF fibroblasts. This is consistent with the finding that genetic manipulations of Slc7a11 in mouse lung fibroblasts had no effect on intracellular $E_{h}(G S H / G S S G)$ in Chapter 2. It was not surprising that regulation of extracellular $E_{h}$ (Cys/CySS) and intracellular $E_{h}(G S H / G S S G)$ were mediated via different mechanisms. In fact, this is a good example to show oxidative stress was not simply overall imbalance between oxidants and antioxidants but disruption of redox regulation network which contains components that are controlled independently of each other.

The regulatory role of SLC7A11 on redox environments is dependent on the transporting activity of system $X_{c}$. Exploration of why SLC7A11 was lower in IPF fibroblast and the relation between SLC7A11 expression and system $X_{c}{ }^{-}$ activity is critical for understanding the pathogenesis of IPF. Studies of regulation of SLC7A11 expression and system $X_{c}{ }^{-}$activity are primarily focused on the transcription level and support the idea that transcriptional regulation of SLC7A11 is much more important for determining system $X_{c}^{-}$activity compared to expression of SLC7A11-interacting heavy chain SLC3A2, which forms multiple transporting systems with different subunits and lacks specificity (263). 
Transcriptional up-regulation of SLC7A11 has been shown to be mediated via 3 transcription factors, nuclear factor, erythroid 2 like 2 (NRF2), activating transcription factor 4 (ATF4) and ETS proto-oncogene 1, transcription factor (ETS-1) (264-266). NRF2 is a master switch for inducing expressions of multiple anti-oxidant genes. Not only SLC7A11, but also GSH metabolismassociated genes, inducing glutamate-cysteine ligase (GCL), glutathione synthetase (GSS), glutathione S-transferase (GST), glutathione peroxidase (GPX), are all induced when NRF2 binds with the anti-oxidant response element (ARE) in the corresponding gene promoter in cell nuclei $(267,268)$. Without cellular stress, NRF2 is ubiquitinated via kelch like ECH associated protein 1/cullin 3/ring-box 1 (KEAP1/CUL3/RBX1) E3-ubiquitin ligase complex and degraded via proteasome (269). ROS, electrophiles and heavy metals have been shown to up-regulate SLC7A11 via disrupting the inhibitory effect of KEAP1 on NRF2 and promoting translocation of NRF2 into the nuclei (270, 271). ATF4 mediates SLC7A11 induction via nutrient deficiency (272). Amino acid deprivation, such as CySS starvation, results in increased number of unloaded t-RNAs (273). Free t-RNAs then activates general control nonderepressible 2 (GCN2), which is a kinase that phosphorylates eukaryotic translation initiation factor $2 \alpha$ (elF2 $\alpha)$ (274). Phosphorylation of elF2 $\alpha$ further promotes translation of ATF4, and ATF4 promotes SLC7A11 transcription via binding to the amino acid response element (AARE) in SLC7A11 promoter region (275). Furthermore, ETS proto-oncogene 1, transcription factor (ETS-1), 
downstream of RAS, increases SLC7A11 expression in synergy with ATF4, suggesting the supportive role of SLC7A11 in RAS-induced tumorigenesis (266).

Other transcription factors can down-regulate SLC7A11 transcription, including p53, ATF3, BTB and CNC homology 1 (BACH1) and POU class 2 homeobox 1 (POU2F1) (276-279). The p53 protein is a famous tumor suppressor that regulates genes associated with cell cycle arrest, apoptosis and senescence (280). Though p53 inhibits SLC7A11 transcription, mutation of p53 does not always accompany over-expression of SLC7A11 in multiple tumors, further supporting the idea that transcription of SLC7A11 is regulated by multiple mechanisms $(249,276,281)$. SLC7A11 is closely associated with ferroptosis, a non-apoptotic and iron-dependent form of regulated cell death resulted from GSH exhaustion and lipid peroxides accumulation (282). ATF3, which is induced by various cellular stresses including DNA damage and oxidative stress, decreases SLC7A11 transcription in a p53-independent way and promotes erastin-induced ferroptosis $(277,283,284)$. BACH1 competes with NRF2 for ARE in promoters of redox associated genes (285). Contrary to NRF2, BACH1 suppresses expressions of those anti-oxidant genes including SLC7A11, stimulating ferroptosis $(279,286)$. POU2F1 also binds with SLC7A11 promoter region and inhibits SLC7A11 transcription, resulting in change of melanin production of melanocytes and change of fur color in rabbits (278).

Deubiquitinases involved in chromatin modification have been shown to 
inhibit SLC7A11 transcription. BRCA1-associated protein 1 (BAP1) is a tumor suppressor and inhibits SLC7A11 expression via decreasing H2A ubiquitination $(287,288)$. Under ferroptotic stress, p53 binds with ubiquitin-specific peptidase 7 (USP7) and promotes USP7 translocation into the nuclei, where USP7 inactivates SLC7A11 transcription via removing monoubiquitination of histone H2B at lysine 120 (H2Bub1) (289). This inhibitory effect of p53 on SLC7A11 expression via epigenetic chromatin regulation is independent of its inhibitory effect as a transcription factor (289).

Chromatin remodeling molecules also influence SLC7A11 transcription. SWI/SNF chromatin-remodeling complex is altered in many cancers (290). ATrich interaction domain $1 \mathrm{~A}(\mathrm{ARID1A})$ gene encodes one component of this complex (291). It enhances the effect of NRF2 on inducing SLC7A11 (292). ARID1A deficiency impairs NRF2 recruitment and NRF-mediated SLC7A11 transcription (292). However, over-expression of NRF2 rescues SLC7A11 expression, indicating ARID1A is not essential for SLC7A11 induction (292).

MicroRNAs (miRNAs) bind with and suppress SLC7A11 mRNA. MiR-26b, miR-27a, miR-126-3p, miR-126-5p, miR-372, miR-373, miR-374b-5p, miR-375, miR-627-5p, miR-3683, miR-4669 and miR-4740-3p have all been shown to target 3'-untranslated region (3'-UTR) of SLC7A11 transcripts to inhibit SLC7A11 mRNA translation or to promote SLC7A11 mRNA degradation (293301).

A variety of endogenous cytokines and exogenous stimulants, such as 
interleukin-1 $\beta$ (IL-1 $\beta)$, tumor necrosis factor $\alpha(\mathrm{TNF} \alpha)$, fibroblasts growth factor 2 (FGF2), erythropoietin (EPO) and bacterial lipopolysaccharide (LPS), increases SLC7A11 transcription (195). However, the mechanisms are not elucidated.

Interactions between SLC7A11 protein and other molecules can increase or decrease the activity of system $\mathrm{X}_{c^{-}}^{-}$, depending on the interactive molecules. EGFR physically interacts with and stabilizes SLC7A11 protein in a kinaseindependent manner in EGFR-expressing glioma cells, which manifest increased CySS uptake and enhanced anti-oxidant capacity (250). CD44 is an adhesion molecule (302). Variant isoform of CD44 (CD44v) is expressed in epithelial tumors (303). CD44v interacts with and stabilizes SLC7A11, maintaining higher intracellular GSH synthesis for survival of gastrointestinal cancer cells (304). CD44v-SLC7A11 interaction is also involved in acquisition of cisplatin-resistance in lung cancer and 5-fluorouracil-resistance in gastric cancer $(305,306)$. CD133, a cancer stem cell marker, is shown to stabilize SLC7A11 and increase survival and drug resistance of liver cancer cells (307). The stability of SLC7A11 also requires the heavy chain SLC3A2 (308). On the contrary, formation of SLC7A11-Beclin 1 (BECN1) complex inhibits system $X_{c}{ }^{-}$ activity $(309,310)$. BECN1 is a key component in the stage of nucleation during autophagy (311). Sulfasalazine and erastin induce phosphorylation and activation of AMP-activated protein kinase (AMPK) (309). AMPK further phosphorylates BECN1 (309). Phosphorylated BECN1 interacts with SLC7A11 
protein directly, resulting in suppression of system $X_{c}^{-}$activity (309). Inhibition of system $\mathrm{X}_{c}{ }^{-}$activity via formation of BECN1-SLC7A11 complex has also been shown to mediate isoflurane-induced toxicity (310).

Post-translational modifications of SLC7A11 also change the activity of system $\mathrm{X}_{c^{-}}$. OTU deubiquitinase, ubiquitin aldehyde binding 1 (OTUB1) directly interacts with SLC7A11 and stabilizes SLC7A11 via deuibiquitination (312). Furthermore, this OTUB1-SLC7A11 interaction is enhanced via overexpression of CD44 (312). On the contrary, mTORC2 interacts with and phosphorylates SLC7A11, resulting in inhibition of system $X_{c^{-}}$activity (313).

Methylation of SLC7A11 DNA is shown to be inversely correlated with alcohol consumption in a study using saliva samples from European American males and another study using blood samples from non-Hispanic white females $(314,315)$.

Whether expression and stability of SLC7A11 are regulated via these or other mechanisms and how activity of system $X_{c}^{-}$change correspondingly remain to be explored in IPF and non-IPF human fibroblasts.

In conclusion, lung fibroblasts from IPF patients have more oxidized extracellular $\mathrm{E}_{\mathrm{h}}$ (Cys/CySS) than lung fibroblasts from non-IPF donors. Lower SLC7A11 could account for the oxidation of extracellular Eh(Cys/CySS) and subsequent increase of pro-fibrotic genes in IPF fibroblasts. Future studies will explore how SLC7A11 is dysregulated in IPF fibroblasts to identify new therapeutic targets for preventing and reversing lung fibrosis. 


\section{CHAPTER V}

\section{SUMMARY AND DISCUSSION}

5.1 Slc7a11 and its role in regulating redox environments for lung fibroblasts

Because of the earlier emergence of oxidation of redox potentials compared to clinical symptoms and the pro-fibrotic effects of such oxidation, it is imperative to explore how redox potential is regulated and whether oxidation could be intervened and reversed. Previous studies have shown Slc7a11 is a candidate for controlling redox potentials.

Aging is associated with oxidation of redox potentials. Plasma $\mathrm{E}_{\mathrm{h}}$ (Cys/CySS) becomes progressively oxidized at a linear rate of $0.16 \mathrm{mV} / \mathrm{year}$ over the entire age span $(136,137)$. Plasma $E_{h}(G S H / G S S G)$ is stable before 45 years old, but gets oxidized at a faster rate of $0.7 \mathrm{mV} /$ year after 45 years old $(136,137)$. Lung fibroblasts isolated from old mice produce a more oxidizing extracellular $E_{h}$ (Cys/CySS) than fibroblasts from young mice, which reproduces age-dependent oxidation of redox environment observed in human plasma (157). Furthermore, the capacity to recover from oxidative challenge is decreased in old fibroblasts as it takes longer time to re-establish the set-point value of extracellular $E_{h}$ (Cys/CySS) for those old fibroblasts (157). Therefore, 
those primary lung fibroblasts from young and old mice provide us with a good in vitro model to explore the regulation of redox potentials.

Concomitant with oxidation of extracellular $\mathrm{E}_{h}(\mathrm{Cys} / \mathrm{CySS})$, the expression of Slc7a11 is 10-fold lower in old fibroblasts than young fibroblasts (157). Slc7a11 is the substrate-specific part of system $X_{c}^{-}$, a CySS/glutamate antiporter located on cell membrane $(172,316)$. System $X_{c}{ }^{-}$is a heterodimer and composed of the light chain Slc7a11 and the heavy chain Slc3a2 (317). The ratio of system $X_{c^{-}}-$mediated CySS import and glutamate export is 1:1 (318). Slc7a11 determines the specific function and activity of system $X_{c^{-}}^{-}(195,319)$. Slc3a2 is also known as CD98 or 4F2 heavy chain and is responsible for trafficking of Slc7a11 to the cell membrane as a chaperone $(320,321)$. Slc3a2 also interacts with other light chains to form other amino acid transporters (322324). Therefore, Slc7a11 plays a more important role in studying regulation of system $\mathrm{X}_{c^{-}}$activity.

Slc7a11 connects the Cys/CySS and GSH/GSSG redox couples (Figure 5.1). After CySS is imported via Slc7a11, it is reduced rapidly to Cys enzymatically by thioredoxin reductase 1 (Txnrd1) or thioredoxin domain containing 17 (Txndc17) or non-enzymatically by GSH because of the much more reducing intracellular environment compared to the extracellular environment $(186,195)$. Cys produced from Slc7a11-mediated CySS import is the major source of cellular Cys, although in several tissues, Cys can be synthesized from homocysteine or methionine via transsulfuration pathway 
$(325,326)$. Cys can be exported by system ASC directly and oxidized back to CySS (327). Cys is also the rate limiting component for intracellular GSH synthesis (188). Glutamate-cysteine ligase (GCL) catalyzes the combination of Cys and glutamate to form $y$-glutamyl cysteine, and glutathione synthetase (GSS) further catalyzes the addition of glycine to Y-glutamyl cysteine to generate GSH (175). GSH is the major small molecular anti-oxidant inside the cells. It reduces various oxidants with or without enzymes, mitigating oxidative stress. GSSG is the oxidative product formed from two molecules of GSH linked by a disulfide. GSSG can be reduced back to GSH by glutathione reductase with electrons from NADPH (328). Both GSH and GSSG can be exported by the multi-drug resistance protein (MRP) (329-331). Outside of the cells, membrane-bound $y$-glutamyl transferase (GGT) breaks down GSH to release glutamate and cysteinyl glycine (332). Dipeptidase then cleaves cysteinyl glycine to produce Cys and glycine (332). Though other transporters are present, Slc7a11-dependent transporting system $X_{c}^{-}$is pivotal for the maintenance of the cycle between Cys/CySS and GSH/GSSG. 


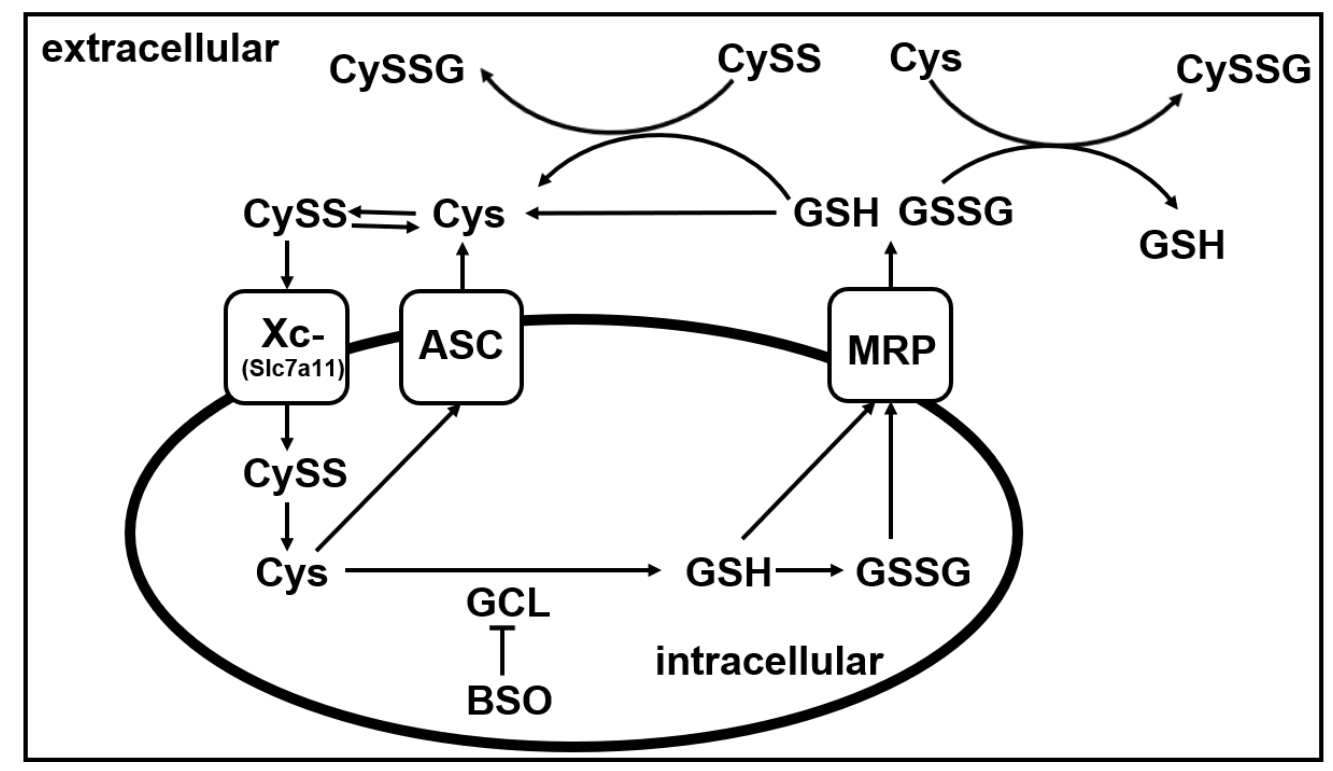

Figure 5.1 Connection between Cys/CySS and GSH/GSSG redox couples. 
The biological roles of SIc7a11 are based on system $X_{c^{-}}$-mediated CySS import and could be mediated by system $\mathrm{X}_{\mathrm{c}}{ }^{-}$-related Cys release. It has been observed that age-dependent oxidation of extracellular $E_{h}$ (Cys/CySS) coincides with age-dependent decrease of Slc7a11 in primary lung fibroblasts, suggesting the potential regulatory role of Slc7a11 on redox potentials (157). Chapter 2 shows Slc7a11 is the key controller of extracellular $E_{h}$ (Cys/CySS) (203). Furthermore, induction or over-expression of Slc7a11 correlates with increase of extracellular Cys concentration, while suppression or knock-down of Slc7a11 correlates with decrease of extracellular Cys concentration (203). Extracellular CySS concentration does not change significantly after SIc7a11 manipulation (203). Those results show although Slc7a11 imports CySS directly, it is the exported Cys that mediates the regulatory effect of Slc7a11 on extracellular $E_{h}($ Cys/CySS). Blocking intracellular GSH synthesis results in dramatic depletion of extracellular GSH concentration without affecting extracellular Cys concentration, indicating GSH synthesis and export might not be essential for regulation of extracellular $E_{h}$ (Cys/CySS) (203). In support of my

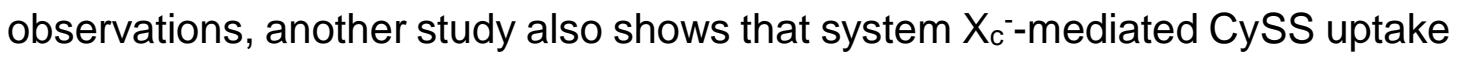
drives Cys release in both vascular smooth muscle cells and endothelial cells (333). Rather than the increased intracellular GSH, the reducing extracellular environment created by system $X_{c^{-}}$-associated Cys release increases lymphoma cell survival (190). Change of extracellular $\mathrm{E}_{h}$ (Cys/CySS) itself also has multiple biological influences, which has been discussed in previous 
paragraphs in Section 1.3. Taken together, one possible mechanism by which Slc7a11 exerts its functions could be directly linked to regulation of Cys/CySS balance and its redox potential without requiring the interaction between Cys/CySS and GSH/GSSG redox couples.

SIc7a11 regulates intracellular redox environments in a GSH-dependent manner, and the effects can also be reflected by multiple peptidyl cysteines. Cysteines are widely distributed among proteins and could be important sites for post-translational modifications $(334,335)$. Cys can be in the reduced form or a variety of oxidized forms (336). Some of the oxidize forms are reversible (e.g. disulfide, sulfinic acid) while some are not (e.g. sulfonic acid) (163). Some thiols exist in the active thiolate form with negative charge while some are not based on local pH (208). Cys can also form coordination compounds with transition metal ions (337). The structural diversity of Cys results in the functional flexibility. Redox state of a specific peptidyl Cys is quantitatively represented by the ratio of the oxidized portion to the reduced portion of this Cys. The results in Chapter 3 show redox states of Cys change spontaneously with aging. Though oxidative stress increases with aging, redox states do not become oxidized for all Cys residues, indicating that in addition to the general oxidation of intracellular redox environment, specific pathways are regulated individually via redox regulation. Slc7a11 decreases aging $(157,203)$. Considering Cys reduced from system $\mathrm{X}_{c}$-imported CySS provides the ratelimiting component for GSH synthesis, decrease of Slc7a11 level could result 
in decrease of intracellular GSH level, disrupting the balance of intracellular redox environments and redox signaling. Many studies have confirmed decrease or Slc7a11 expression or inhibition of system $X_{c}{ }^{-}$activity results in decrease of GSH, rendering cells more susceptible to death (338-340). However, the downstream effects on redox states of Cys and redox signaling are less studied. My studies fill the gap and indicate that over-expression of SIc7a11 might compensate for its age-dependent deficiency and re-establish the optimal homeostasis of redox environments. Furthermore, restoration of intracellular redox environment further results in reverse of Cys redox states and redox signaling, at least partially. In Chapter 3, I have found the most affected age-dependent Slc7a11-responsive signaling pathways are eukaryotic translation initiation factor 2 (EIF2) signaling, actin cytoskeleton and integrinlinked kinase (ILK) signaling and protein ubiquitination pathway. These 3 pathways demonstrate that peptidyl Cys residues whose redox states change with aging but are reversible by Slc7a11 over-expression are enriched in basic biological activities including protein turnover and cytoskeleton dynamics.

Mechanisms responsible for regulating redox environments are altered during IPF pathogenesis. Chapter 4 shows human lung fibroblasts from IPF patients produce a more oxidizing extracellular $\mathrm{En}_{\mathrm{h}}(\mathrm{Cys} / \mathrm{CySS})$ than fibroblasts from non-IPF donors. This is consistent with my observation that plasma $\mathrm{E}_{\mathrm{h}}$ (Cys/CySS) of IPF patients is more oxidized than that of non-IPF donors. Considering Slc7a11 is the key regulator of extracellular Eh(Cys/CySS) in 
primary mouse lung fibroblasts, I hypothesize Slc7a11 could have similar roles in human lung fibroblasts. However, higher Slc7a11 expression only correlates with more reduced extracellular $E_{h}$ (Cys/CySS) in non-IPF fibroblasts, while this correlation is impaired in IPF fibroblasts. Furthermore, the correlation between Slc7a11 expression and intracellular $\mathrm{Eh}_{\mathrm{h}}(\mathrm{GSH} / \mathrm{GSSG})$ in non-IPF fibroblasts is also disrupted in IPF fibroblasts. Those results indicate Slc7a11 might lose its role of mediating the cycle between Cys/CySS and GSH/GSSG redox couples during IPF development.

\subsection{Strengths and weaknesses}

Studies in this dissertation focus on regulation of both extracellular and intracellular redox environments by fibroblasts. The interaction between fibroblasts and redox environments are comparable to that between fibroblasts and matrix proteins. Oxidation of extracellular environments stimulates profibrotic responses of fibroblasts while fibroblasts are able to regulate their redox environments. However, the regulatory capability was impaired with aging, the most important risk factor of IPF, and altered during IPF fibrogenesis. Change of redox environments could occur even before the appearance of irreversible fibrosis, indicating potential clinical significance for early intervention to halt or even reverse fibrosis. My model of lung fibroblasts from young and old mice simulates the effects of aging in vitro and allows us to explore why the ability of maintaining optimal redox environments is decreased with aging and what is the key controller of redox environments for fibroblasts. The young and old 
fibroblasts were isolated from healthy mice. The studies in this dissertation tried to uncover how the susceptibility increases before the actual fibrosis happens. Thus, it is the initial step towards finding novel targets for early intervention to decrease IPF susceptibility. This dissertation also begins to examine whether mouse and human fibroblasts share similar regulatory mechanisms of redox environments and compare IPF and non-IPF fibroblasts. This is the further step towards revealing whether actual redox environments and their regulatory mechanisms are altered in the diseased status of IPF. This is the foundation of developing targets for future clinical trials.

There are several limitations. First, fibroblasts are not the only cells involved in IPF pathogenesis. Epithelial cells, inflammatory cells, endothelial cells and pericytes might all have different and complementary roles to play. Their exact contributions to IPF are not fully elucidated. Whether and how those cell types contribute to regulation of redox environments and whether there are interactions between fibroblasts and other cell types remain unexplored. Second, the discrepancies of redox regulation mechanism between IPF and non-IPF fibroblasts could be attributed to the small sample size. Fibroblasts from only 6 IPF patients and 6 non-IPF donors are available. It is unknown whether adding more samples will change the conclusion. Last, confirmation of the changes of redox states of peptidyl cysteines in the age-dependent Slc7a11-responsive pathways are needed as redox proteomic assays are not as accurate as individual experiments targeting specific proteins. 


\subsection{Future directions}

5.3.1 What is the role of Slc7a11 in mediating the anti-fibrotic effects of sulforaphane in bleomycin-induced lung fibrosis mouse model?

This dissertation focuses on exploring the role of Slc7a11 in regulating extracellular and intracellular redox environments mouse and human lung fibroblasts in vitro. In vivo experiments using wild-type and Slc7a11 knock-out mice will further clarify the role of Slc7a11. Sulforaphane is used in Chapter 2 to induce Slc7a11 expression. Sulforaphane has also been shown to decrease mRNA expression of pro-fibrotic genes and deposition of collagen, mitigating bleomycin-induced lung fibrosis in mice (341). It will be interesting to explore whether Slc7a11 mediates these anti-fibrotic effects of sulforaphane.

5.3.2 How expression of Slc7a11 is regulated with chronological aging?

Chapter 2 shows Slc7a11 expression is lower in fibroblasts isolated from old mice compared to young mice. Why aging results in decrease of Slc7a11 remains to be explored. Chapter 4 includes summaries of transcriptional and post-transcriptional regulation of Slc7a11 expression and post-translational regulation of Slc7a11 activity. Whether those mechanisms are responsible for the age-dependent decrease of Slc7a11 in primary mouse lung fibroblasts and whether there are other mechanisms involved remain to be explored.

5.3.3 Is there a connection between cellular senescence and Slc7a11 expression? How do they relate to each other?

Fibroblasts isolated from IPF patients display multiple characteristics of 
cellular senescence (29). Chapter 3 shows Slc7a11 restores age-dependent changes of redox potentials of many proteins. It will be interesting to explore whether Slc7a11 manipulations could reverse changes of senescent markers. If Slc7a11 could indeed affect the process of aging, it will be of great value to study how such effect is connected to the susceptibility of IPF. 


\section{REFERENCES}

1. Martinez FJ, Collard HR, Pardo A, Raghu G, Richeldi L, Selman M, et al. Idiopathic pulmonary fibrosis. Nature reviews Disease primers. 2017;3:17074. 2. Raghu G, Collard HR, Egan JJ, Martinez FJ, Behr J, Brown KK, et al. An official ATS/ERS/JRS/ALAT statement: idiopathic pulmonary fibrosis: evidencebased guidelines for diagnosis and management. American journal of respiratory and critical care medicine. 2011;183(6):788-824.

3. Raghu G, Remy-Jardin M, Myers JL, Richeldi L, Ryerson CJ, Lederer DJ, et al. Diagnosis of Idiopathic Pulmonary Fibrosis. An Official ATS/ERS/JRS/ALAT Clinical Practice Guideline. American journal of respiratory and critical care medicine. 2018;198(5):e44-e68.

4. Larsen BT, Colby TV. Update for pathologists on idiopathic interstitial pneumonias. Archives of pathology \& laboratory medicine. 2012;136(10):123441.

5. Katzenstein AL, Mukhopadhyay S, Myers JL. Diagnosis of usual interstitial pneumonia and distinction from other fibrosing interstitial lung diseases. Human pathology. 2008;39(9):1275-94.

6. Ley B, Collard HR. Epidemiology of idiopathic pulmonary fibrosis. Clinical epidemiology. 2013;5:483-92 
7. Raghu G, Chen SY, Yeh WS, Maroni B, Li Q, Lee YC, et al. Idiopathic pulmonary fibrosis in US Medicare beneficiaries aged 65 years and older: incidence, prevalence, and survival, 2001-11. The Lancet Respiratory medicine. 2014;2(7):566-72.

8. Raghu G, Chen SY, Hou Q, Yeh WS, Collard HR. Incidence and prevalence of idiopathic pulmonary fibrosis in US adults 18-64 years old. The European respiratory journal. 2016;48(1):179-86.

9. Raghu G, Weycker D, Edelsberg J, Bradford WZ, Oster G. Incidence and prevalence of idiopathic pulmonary fibrosis. American journal of respiratory and critical care medicine. 2006;174(7):810-6.

10. King TE, Jr., Pardo A, Selman M. Idiopathic pulmonary fibrosis. Lancet (London, England). 2011;378(9807):1949-61 .

11. Olson AL, Swigris JJ, Lezotte DC, Norris JM, Wilson CG, Brown KK. Mortality from pulmonary fibrosis increased in the United States from 1992 to 2003. American journal of respiratory and critical care medicine. 2007;176(3):277-84.

12. Nathan SD, Shlobin OA, Weir N, Ahmad S, Kaldjob JM, Battle E, et al. Long-term course and prognosis of idiopathic pulmonary fibrosis in the new millennium. Chest. 2011;140(1):221-9.

13. Vancheri C, Failla M, Crimi N, Raghu G. Idiopathic pulmonary fibrosis: a disease with similarities and links to cancer biology. The European respiratory journal. 2010;35(3):496-504. 
14. Lynch DA, Sverzellati N, Travis WD, Brown KK, Colby TV, Galvin JR, et al. Diagnostic criteria for idiopathic pulmonary fibrosis: a Fleischner Society White Paper. The Lancet Respiratory medicine. 2018;6(2):138-53.

15. Rochester CL, Vogiatzis I, Holland AE, Lareau SC, Marciniuk DD, Puhan MA, et al. An Official American Thoracic Society/European Respiratory Society Policy Statement: Enhancing Implementation, Use, and Delivery of Pulmonary Rehabilitation. American journal of respiratory and critical care medicine. 2015;192(11):1373-86.

16. Dowman L, Hill CJ, Holland AE. Pulmonary rehabilitation for interstitial lung disease. The Cochrane database of systematic reviews. 2014(10):Cd006322. 17. Khor YH, Goh NSL, McDonald CF, Holland AE. Oxygen Therapy for Interstitial Lung Disease. A Mismatch between Patient Expectations and Experiences. Annals of the American Thoracic Society. 2017;14(6):888-95.

18. King TE, Jr., Bradford WZ, Castro-Bernardini S, Fagan EA, Glaspole I, Glassberg MK, et al. A phase 3 trial of pirfenidone in patients with idiopathic pulmonary fibrosis. The New England journal of medicine. 2014;370(22):208392.

19. Richeldi L, du Bois RM, Raghu G, Azuma A, Brown KK, Costabel U, et al. Efficacy and safety of nintedanib in idiopathic pulmonary fibrosis. The New England journal of medicine. 2014;370(22):2071-82.

20. Raghu G, Rochwerg B, Zhang Y, Garcia CA, Azuma A, Behr J, et al. An Official ATS/ERS/JRS/ALAT Clinical Practice Guideline: Treatment of Idiopathic 
Pulmonary Fibrosis. An Update of the 2011 Clinical Practice Guideline. American journal of respiratory and critical care medicine. 2015;192(2):e3-19. 21. Seibold MA, Wise AL, Speer MC, Steele MP, Brown KK, Loyd JE, et al. A common MUC5B promoter polymorphism and pulmonary fibrosis. The New England journal of medicine. 2011;364(16):1503-12.

22. Lee HL, Ryu JH, Wittmer MH, Hartman TE, Lymp JF, Tazelaar HD, et al. Familial idiopathic pulmonary fibrosis: clinical features and outcome. Chest. 2005;127(6):2034-41.

23. Wang Y, Kuan PJ, Xing C, Cronkhite JT, Torres F, Rosenblatt RL, et al. Genetic defects in surfactant protein A2 are associated with pulmonary fibrosis and lung cancer. American journal of human genetics. 2009;84(1):52-9.

24. Ono S, Tanaka T, Ishida M, Kinoshita A, Fukuoka J, Takaki M, et al. Surfactant protein C G100S mutation causes familial pulmonary fibrosis in Japanese kindred. The European respiratory journal. 2011;38(4):861-9.

25. Tsakiri KD, Cronkhite JT, Kuan PJ, Xing C, Raghu G, Weissler JC, et al. Adult-onset pulmonary fibrosis caused by mutations in telomerase. Proceedings of the National Academy of Sciences of the United States of America. 2007;104(18):7552-7.

26. Oldham JM, Ma SF, Martinez FJ, Anstrom KJ, Raghu G, Schwartz DA, et al. TOLLIP, MUC5B, and the Response to N-Acetylcysteine among Individuals with Idiopathic Pulmonary Fibrosis. American journal of respiratory and critical care medicine. 2015;192(12):1475-82. 
27. Lopez-Otin C, Blasco MA, Partridge L, Serrano M, Kroemer G. The hallmarks of aging. Cell. 2013;153(6):1194-217.

28. Thannickal VJ. Mechanistic links between aging and lung fibrosis. Biogerontology. 2013;14(6):609-15.

29. Alvarez D, Cardenes N, Sellares J, Bueno M, Corey C, Hanumanthu VS, et al. IPF lung fibroblasts have a senescent phenotype. American journal of physiology Lung cellular and molecular physiology. 2017;313(6):L1164-I73.

30. Sueblinvong V, Neujahr DC, Mills ST, Roser-Page S, Ritzenthaler JD, Guidot D, et al. Predisposition for disrepair in the aged lung. The American journal of the medical sciences. 2012;344(1):41-51.

31. Taskar VS, Coultas DB. Is idiopathic pulmonary fibrosis an environmental disease? Proceedings of the American Thoracic Society. 2006;3(4):293-8.

32. Oh CK, Murray LA, Molfino NA. Smoking and idiopathic pulmonary fibrosis. Pulmonary medicine. 2012;2012:808260.

33. Zaman T, Lee JS. Risk factors for the development of idiopathic pulmonary fibrosis: A review. Current pulmonology reports. 2018;7(4):118-25.

34. Fois AG, Paliogiannis $P$, Sotgia $S$, Mangoni AA, Zinellu E, Pirina $P$, et al. Evaluation of oxidative stress biomarkers in idiopathic pulmonary fibrosis and therapeutic applications: a systematic review. Respiratory research. 2018;19(1):51 .

35. Chilosi M, Doglioni C, Murer B, Poletti V. Epithelial stem cell exhaustion in the pathogenesis of idiopathic pulmonary fibrosis. Sarcoidosis, vasculitis, and 
diffuse lung diseases : official journal of WASOG. 2010;27(1):7-18.

36. Parimon T, Yao C, Habiel DM, Ge L, Bora SA, Brauer R, et al. Syndecan-1 promotes lung fibrosis by regulating epithelial reprogramming through extracellular vesicles. JCl insight. 2019;5.

37. Selman M, Pardo A. Role of epithelial cells in idiopathic pulmonary fibrosis: from innocent targets to serial killers. Proceedings of the American Thoracic Society. 2006;3(4):364-72.

38. Konigshoff M, Balsara N, Pfaff EM, Kramer M, Chrobak I, Seeger W, et al. Functional Wnt signaling is increased in idiopathic pulmonary fibrosis. PloS one. 2008;3(5):e2142.

39. Bolanos AL, Milla CM, Lira JC, Ramirez R, Checa M, Barrera L, et al. Role of Sonic Hedgehog in idiopathic pulmonary fibrosis. American journal of physiology Lung cellular and molecular physiology. 2012;303(11):L978-90.

40. Agostini C, Gurrieri C. Chemokine/cytokine cocktail in idiopathic pulmonary fibrosis. Proceedings of the American Thoracic Society. 2006;3(4):357-63.

41. Crooks MG, Hart SP. Coagulation and anticoagulation in idiopathic pulmonary fibrosis. European respiratory review : an official journal of the European Respiratory Society. 2015;24(137):392-9.

42. Zhong Q, Zhou B, Ann DK, Minoo P, Liu Y, Banfalvi A, et al. Role of endoplasmic reticulum stress in epithelial-mesenchymal transition of alveolar epithelial cells: effects of misfolded surfactant protein. American journal of respiratory cell and molecular biology. 2011;45(3):498-509. 
43. Camelo A, Dunmore R, Sleeman MA, Clarke DL. The epithelium in idiopathic pulmonary fibrosis: breaking the barrier. Frontiers in pharmacology. 2014;4:173.

44. Raghu G, Anstrom KJ, King TE, Jr., Lasky JA, Martinez FJ. Prednisone, azathioprine, and $\mathrm{N}$-acetylcysteine for pulmonary fibrosis. The New England journal of medicine. 2012;366(21):1968-77.

45. Desai O, Winkler J, Minasyan M, Herzog EL. The Role of Immune and Inflammatory Cells in Idiopathic Pulmonary Fibrosis. Frontiers in medicine. 2018;5:43.

46. Waters DW, Blokland KEC, Pathinayake PS, Burgess JK, Mutsaers SE, Prele CM, et al. Fibroblast senescence in the pathology of idiopathic pulmonary fibrosis. American journal of physiology Lung cellular and molecular physiology. 2018;315(2):L162-I72.

47. Darby IA, Zakuan N, Billet F, Desmouliere A. The myofibroblast, a key cell in normal and pathological tissue repair. Cellular and molecular life sciences : CMLS. 2016;73(6):1145-57.

48. Habiel DM, Hogaboam CM. Heterogeneity of Fibroblasts and Myofibroblasts in Pulmonary Fibrosis. Current pathobiology reports. 2017;5(2):101-10.

49. Moore MW, Herzog EL. Regulation and Relevance of Myofibroblast Responses in Idiopathic Pulmonary Fibrosis. Current pathobiology reports. 2013;1(3):199-208. 
50. Kendall RT, Feghali-Bostwick CA. Fibroblasts in fibrosis: novel roles and mediators. Frontiers in pharmacology. 2014;5:123.

51. Horowitz JC, Lee DY, Waghray M, Keshamouni VG, Thomas PE, Zhang H, et al. Activation of the pro-survival phosphatidylinositol 3-kinase/AKT pathway by transforming growth factor-beta1 in mesenchymal cells is mediated by p38 MAPK-dependent induction of an autocrine growth factor. The Journal of biological chemistry. 2004;279(2):1359-67.

52. Thannickal VJ, Lee DY, White ES, Cui Z, Larios JM, Chacon R, et al. Myofibroblast differentiation by transforming growth factor-beta1 is dependent on cell adhesion and integrin signaling via focal adhesion kinase. The Journal of biological chemistry. 2003;278(14):12384-9.

53. Liu F, Mih JD, Shea BS, Kho AT, Sharif AS, Tager AM, et al. Feedback amplification of fibrosis through matrix stiffening and COX-2 suppression. The Journal of cell biology. 2010;190(4):693-706.

54. Tashiro J, Rubio GA, Limper AH, Williams K, Elliot SJ, Ninou I, et al. Exploring Animal Models That Resemble Idiopathic Pulmonary Fibrosis. Frontiers in medicine. 2017;4:118.

55. Mouratis MA, Aidinis V. Modeling pulmonary fibrosis with bleomycin. Current opinion in pulmonary medicine. 2011;17(5):355-61.

56. Moore BB, Hogaboam CM. Murine models of pulmonary fibrosis. American journal of physiology Lung cellular and molecular physiology. 2008;294(2):L152-60. 
57. Iyer SS, Ramirez AM, Ritzenthaler JD, Torres-Gonzalez E, Roser-Page S, Mora $\mathrm{AL}$, et al. Oxidation of extracellular cysteine/cystine redox state in bleomycin-induced lung fibrosis. American journal of physiology Lung cellular and molecular physiology. 2009;296(1):L37-45.

58. Carrington R, Jordan S, Pitchford SC, Page CP. Use of animal models in IPF research. Pulmonary pharmacology \& therapeutics. 2018;51:73-8.

59. Sies H. Oxidative stress: oxidants and antioxidants. Experimental physiology. 1997;82(2):291-5.

60. Veith C, Boots AW, Idris M, van Schooten FJ, van der Vliet A. Redox Imbalance in Idiopathic Pulmonary Fibrosis: A Role for Oxidant Cross-Talk Between NADPH Oxidase Enzymes and Mitochondria. Antioxidants \& redox signaling. 2019;31(14):1092-115.

61. Cantin AM, North SL, Fells GA, Hubbard RC, Crystal RG. Oxidantmediated epithelial cell injury in idiopathic pulmonary fibrosis. The Journal of clinical investigation. 1987;79(6):1665-73.

62. Psathakis K, Mermigkis D, Papatheodorou G, Loukides S, Panagou P, Polychronopoulos V, et al. Exhaled markers of oxidative stress in idiopathic pulmonary fibrosis. European journal of clinical investigation. 2006;36(5):3627.

63. Daniil ZD, Papageorgiou E, Koutsokera A, Kostikas K, Kiropoulos T, Papaioannou Al, et al. Serum levels of oxidative stress as a marker of disease severity in idiopathic pulmonary fibrosis. Pulmonary pharmacology \& 
therapeutics. 2008;21(1):26-31.

64. Matsuzawa Y, Kawashima T, Kuwabara R, Hayakawa S, Irie T, Yoshida T, et al. Change in serum marker of oxidative stress in the progression of idiopathic pulmonary fibrosis. Pulmonary pharmacology \& therapeutics. 2015;32:1-6.

65. Montuschi P, Ciabattoni G, Paredi P, Pantelidis P, du Bois RM, Kharitonov SA, et al. 8-Isoprostane as a biomarker of oxidative stress in interstitial lung diseases. American journal of respiratory and critical care medicine. 1998;158(5 Pt 1):1524-7.

66. Chow S, Thomas PS, Malouf M, Yates DH. Exhaled breath condensate (EBC) biomarkers in pulmonary fibrosis. Journal of breath research. 2012;6(1):016004.

67. Jackson R, Ramos C, Gupta C, Gomez-Marin O. Exercise decreases plasma antioxidant capacity and increases urinary isoprostanes of IPF patients. Respiratory medicine. 2010;104(12):1919-28.

68. Malli F, Bardaka F, Tsilioni I, Karetsi E, Gourgoulianis KI, Daniil Z. 8isoprostane levels in serum and bronchoalveolar lavage in idiopathic pulmonary fibrosis and sarcoidosis. Food and chemical toxicology : an international journal published for the British Industrial Biological Research Association. 2013;61:160-3.

69. Rottoli P, Magi B, Cianti R, Bargagli E, Vagaggini C, Nikiforakis N, et al. Carbonylated proteins in bronchoalveolar lavage of patients with sarcoidosis, 
pulmonary fibrosis associated with systemic sclerosis and idiopathic pulmonary fibrosis. Proteomics. 2005;5(10):2612-8.

70. Bargagli E, Penza F, Vagaggini C, Magi B, Perari MG, Rottoli P. Analysis of carbonylated proteins in bronchoalveolar lavage of patients with diffuse lung diseases. Lung. 2007;185(3):139-44.

71. Saleh D, Barnes PJ, Giaid A. Increased production of the potent oxidant peroxynitrite in the lungs of patients with idiopathic pulmonary fibrosis. American journal of respiratory and critical care medicine. 1997;155(5):1763-9. 72. Kanoh S, Kobayashi H, Motoyoshi K. Exhaled ethane: an in vivo biomarker of lipid peroxidation in interstitial lung diseases. Chest. 2005;128(4):2387-92.

73. Kuwano K, Nakashima N, Inoshima I, Hagimoto N, Fujita M, Yoshimi M, et al. Oxidative stress in lung epithelial cells from patients with idiopathic interstitial pneumonias. The European respiratory journal. 2003;21(2):232-40.

74. Rahman I, Skwarska E, Henry M, Davis M, O'Connor CM, FitzGerald MX, et al. Systemic and pulmonary oxidative stress in idiopathic pulmonary fibrosis. Free radical biology \& medicine. 1999;27(1-2):60-8.

75. Veith C, Drent M, Bast A, van Schooten FJ, Boots AW. The disturbed redoxbalance in pulmonary fibrosis is modulated by the plant flavonoid quercetin. Toxicology and applied pharmacology. 2017;336:40-8.

76. Markart P, Luboeinski T, Korfei M, Schmidt R, Wygrecka M, Mahavadi P, et al. Alveolar oxidative stress is associated with elevated levels of nonenzymatic low-molecular-weight antioxidants in patients with different forms of chronic 
fibrosing interstitial lung diseases. Antioxidants \& redox signaling. 2009;11(2):227-40.

77. Muramatsu Y, Sugino K, Ishida F, Tatebe J, Morita T, Homma S. Effect of inhaled $\mathrm{N}$-acetylcysteine monotherapy on lung function and redox balance in idiopathic pulmonary fibrosis. Respiratory investigation. 2016;54(3):170-8.

78. Cantin AM, Hubbard RC, Crystal RG. Glutathione deficiency in the epithelial lining fluid of the lower respiratory tract in idiopathic pulmonary fibrosis. The American review of respiratory disease. 1989;139(2):370-2.

79. Meyer A, Buhl R, Magnussen $\mathrm{H}$. The effect of oral $\mathrm{N}$-acetylcysteine on lung glutathione levels in idiopathic pulmonary fibrosis. The European respiratory journal. 1994;7(3):431-6.

80. Beeh KM, Beier J, Haas IC, Kornmann O, Micke P, Buhl R. Glutathione deficiency of the lower respiratory tract in patients with idiopathic pulmonary fibrosis. The European respiratory journal. 2002;19(6):1119-23.

81. Kang YP, Lee SB, Lee JM, Kim HM, Hong JY, Lee WJ, et al. Metabolic Profiling Regarding Pathogenesis of Idiopathic Pulmonary Fibrosis. Journal of proteome research. 2016;15(5):1717-24.

82. Odajima N, Betsuyaku T, Nagai K, Moriyama C, Wang DH, Takigawa T, et al. The role of catalase in pulmonary fibrosis. Respiratory research. 2010;11:183.

83. Schamberger AC, Schiller HB, Fernandez IE, Sterclova M, Heinzelmann K, Hennen E, et al. Glutathione peroxidase 3 localizes to the epithelial lining fluid 
and the extracellular matrix in interstitial lung disease. Scientific reports. 2016;6:29952.

84. Kinnula VL, Hodgson UA, Lakari EK, Tan RJ, Sormunen RT, Soini YM, et al. Extracellular superoxide dismutase has a highly specific localization in idiopathic pulmonary fibrosis/usual interstitial pneumonia. Histopathology. 2006;49(1):66-74.

85. Borzi RM, Grigolo B, Meliconi R, Fasano L, Sturani C, Fabbri M, et al. Elevated serum superoxide dismutase levels correlate with disease severity and neutrophil degranulation in idiopathic pulmonary fibrosis. Clinical science (London, England : 1979). 1993;85(3):353-9.

86. Anathy V, Lahue KG, Chapman DG, Chia SB, Casey DT, Aboushousha R, et al. Reducing protein oxidation reverses lung fibrosis. Nature medicine. 2018;24(8):1128-35.

87. Watson WH, Yang X, Choi YE, Jones DP, Kehrer JP. Thioredoxin and its role in toxicology. Toxicological sciences : an official journal of the Society of Toxicology. 2004;78(1):3-14.

88. Tiitto L, Kaarteenaho-Wiik R, Sormunen R, Holmgren A, Paakko P, Soini Y, et al. Expression of the thioredoxin system in interstitial lung disease. The Journal of pathology. 2003;201(3):363-70.

89. Vuorinen K, Ohlmeier S, Lepparanta O, Salmenkivi K, Myllarniemi M, Kinnula VL. Peroxiredoxin II expression and its association with oxidative stress and cell proliferation in human idiopathic pulmonary fibrosis. The journal of 
histochemistry and cytochemistry : official journal of the Histochemistry Society. 2008;56(10):951-9.

90. Mazur W, Lindholm P, Vuorinen K, Myllarniemi M, Salmenkivi K, Kinnula VL. Cell-specific elevation of NRF2 and sulfiredoxin-1 as markers of oxidative stress in the lungs of idiopathic pulmonary fibrosis and non-specific interstitial pneumonia. APMIS : acta pathologica, microbiologica, et immunologica Scandinavica. 2010;118(9):703-12.

91. Nicolussi A, D'Inzeo S, Capalbo C, Giannini G, Coppa A. The role of peroxiredoxins in cancer. Molecular and clinical oncology. 2017;6(2):139-53.

92. Ramesh A, Varghese SS, Doraiswamy J, Malaiappan S. Role of sulfiredoxin in systemic diseases influenced by oxidative stress. Redox biology. 2014;2:1023-8.

93. Jeong W, Bae SH, Toledano MB, Rhee SG. Role of sulfiredoxin as a regulator of peroxiredoxin function and regulation of its expression. Free radical biology \& medicine. 2012;53(3):447-56.

94. Hanschmann EM, Godoy JR, Berndt C, Hudemann C, Lillig CH. Thioredoxins, glutaredoxins, and peroxiredoxins--molecular mechanisms and health significance: from cofactors to antioxidants to redox signaling. Antioxidants \& redox signaling. 2013;19(13):1539-605.

95. Hecker L, Cheng J, Thannickal VJ. Targeting NOX enzymes in pulmonary fibrosis. Cellular and molecular life sciences : CMLS. 2012;69(14):2365-71.

96. Hecker L, Logsdon NJ, Kurundkar D, Kurundkar A, Bernard K, Hock T, et 
al. Reversal of persistent fibrosis in aging by targeting Nox4-Nrf2 redox imbalance. Science translational medicine. 2014;6(231):231ra47.

97. Pache JC, Carnesecchi S, Deffert C, Donati Y, Herrmann FR, BarazzoneArgiroffo $\mathrm{C}$, et al. NOX-4 is expressed in thickened pulmonary arteries in idiopathic pulmonary fibrosis. Nature medicine. 2011;17(1):31-2; author reply 2-3.

98. Hara H, Kuwano K, Araya J. Mitochondrial Quality Control in COPD and IPF. Cells. 2018;7(8).

99. Liu Y, Fiskum G, Schubert D. Generation of reactive oxygen species by the mitochondrial electron transport chain. Journal of neurochemistry. 2002;80(5):780-7.

100. Liu RM, Gaston Pravia KA. Oxidative stress and glutathione in TGFbeta-mediated fibrogenesis. Free radical biology \& medicine. 2010;48(1):1-15. 101. Krstic J, Trivanovic D, Mojsilovic S, Santibanez JF. Transforming Growth Factor-Beta and Oxidative Stress Interplay: Implications in Tumorigenesis and Cancer Progression. Oxidative medicine and cellular longevity. 2015;2015:654594.

102. Liu RM, Desai LP. Reciprocal regulation of TGF-beta and reactive oxygen species: A perverse cycle for fibrosis. Redox biology. 2015;6:565-77.

103. Oruqaj G, Karnati S, Vijayan V, Kotarkonda LK, Boateng E, Zhang W, et al. Compromised peroxisomes in idiopathic pulmonary fibrosis, a vicious cycle inducing a higher fibrotic response via TGF-beta signaling. Proceedings 
of the National Academy of Sciences of the United States of America. 2015;112(16):E2048-57.

104. Bellocq A, Azoulay E, Marullo S, Flahault A, Fouqueray B, Philippe C, et al. Reactive oxygen and nitrogen intermediates increase transforming growth factor-beta1 release from human epithelial alveolar cells through two different mechanisms. American journal of respiratory cell and molecular biology. 1999;21(1):128-36.

105. Amara N, Goven D, Prost F, Muloway R, Crestani B, Boczkowski J. NOX4/NADPH oxidase expression is increased in pulmonary fibroblasts from patients with idiopathic pulmonary fibrosis and mediates TGFbeta1-induced fibroblast differentiation into myofibroblasts. Thorax. 2010;65(8):733-8.

106. Zank DC, Bueno M, Mora AL, Rojas M. Idiopathic Pulmonary Fibrosis: Aging, Mitochondrial Dysfunction, and Cellular Bioenergetics. Frontiers in medicine. 2018;5:10.

107. Liu RM, Vayalil PK, Ballinger C, Dickinson DA, Huang WT, Wang S, et al. Transforming growth factor beta suppresses glutamate-cysteine ligase gene expression and induces oxidative stress in a lung fibrosis model. Free radical biology \& medicine. 2012;53(3):554-63.

108. Peltoniemi M, Kaarteenaho-Wiik R, Saily M, Sormunen R, Paakko P, Holmgren A, et al. Expression of glutaredoxin is highly cell specific in human lung and is decreased by transforming growth factor-beta in vitro and in interstitial lung diseases in vivo. Human pathology. 2004;35(8):1000-7. 
109. Michaeloudes C, Sukkar MB, Khorasani NM, Bhavsar PK, Chung KF. TGF-beta regulates Nox4, MnSOD and catalase expression, and IL-6 release in airway smooth muscle cells. American journal of physiology Lung cellular and molecular physiology. 2011;300(2):L295-304.

110. Ghatak S, Hascall VC, Markwald RR, Feghali-Bostwick C, Artlett CM, Gooz M, et al. Transforming growth factor beta1 (TGFbeta1)-induced CD44V6NOX4 signaling in pathogenesis of idiopathic pulmonary fibrosis. The Journal of biological chemistry. 2017;292(25):10490-519.

111. Schuliga M, Pechkovsky DV, Read J, Waters DW, Blokland KEC, Reid AT, et al. Mitochondrial dysfunction contributes to the senescent phenotype of IPF lung fibroblasts. Journal of cellular and molecular medicine. 2018;22(12):5847-61.

112. Davalli P, Mitic T, Caporali A, Lauriola A, D'Arca D. ROS, Cell Senescence, and Novel Molecular Mechanisms in Aging and Age-Related Diseases. Oxidative medicine and cellular longevity. 2016;2016:3565127.

113. Colavitti R, Finkel T. Reactive oxygen species as mediators of cellular senescence. IUBMB life. 2005;57(4-5):277-81.

114. Jain M, Rivera S, Monclus EA, Synenki L, Zirk A, Eisenbart J, et al. Mitochondrial reactive oxygen species regulate transforming growth factor-beta signaling. The Journal of biological chemistry. 2013;288(2):770-7.

115. Bocchino M, Agnese S, Fagone E, Svegliati S, Grieco D, Vancheri C, et al. Reactive oxygen species are required for maintenance and differentiation 
of primary lung fibroblasts in idiopathic pulmonary fibrosis. PloS one. 2010;5(11):e14003.

116. Gorowiec MR, Borthwick LA, Parker SM, Kirby JA, Saretzki GC, Fisher AJ. Free radical generation induces epithelial-to-mesenchymal transition in lung epithelium via a TGF-beta1-dependent mechanism. Free radical biology \& medicine. 2012;52(6):1024-32.

117. He C, Ryan AJ, Murthy S, Carter AB. Accelerated development of pulmonary fibrosis via $\mathrm{Cu}, \mathrm{Zn}$-superoxide dismutase-induced alternative activation of macrophages. The Journal of biological chemistry. 2013;288(28):20745-57.

118. Waghray M, Cui Z, Horowitz JC, Subramanian IM, Martinez FJ, Toews $\mathrm{GB}$, et al. Hydrogen peroxide is a diffusible paracrine signal for the induction of epithelial cell death by activated myofibroblasts. FASEB journal : official publication of the Federation of American Societies for Experimental Biology. 2005;19(7):854-6.

119. Geiser T, Ishigaki M, van Leer C, Matthay MA, Broaddus VC. $H(2) O(2)$ inhibits alveolar epithelial wound repair in vitro by induction of apoptosis. American journal of physiology Lung cellular and molecular physiology. 2004;287(2):L448-53.

120. Andersson-Sjoland A, Karlsson JC, Rydell-Tormanen K. ROS-induced endothelial stress contributes to pulmonary fibrosis through pericytes and Wnt signaling. Laboratory investigation; a journal of technical methods and 
pathology. 2016;96(2):206-17.

121. Kliment CR, Oury TD. Oxidative stress, extracellular matrix targets, and idiopathic pulmonary fibrosis. Free radical biology \& medicine. 2010;49(5):70717.

122. Bjermer L, Lundgren R, Hallgren R. Hyaluronan and type III procollagen peptide concentrations in bronchoalveolar lavage fluid in idiopathic pulmonary fibrosis. Thorax. 1989;44(2):126-31.

123. Gao F, Koenitzer JR, Tobolewski JM, Jiang D, Liang J, Noble PW, et al. Extracellular superoxide dismutase inhibits inflammation by preventing oxidative fragmentation of hyaluronan. The Journal of biological chemistry. 2008;283(10):6058-66 .

124. Kliment CR, Englert JM, Gochuico BR, Yu G, Kaminski N, Rosas I, et al. Oxidative stress alters syndecan-1 distribution in lungs with pulmonary fibrosis. The Journal of biological chemistry. 2009;284(6):3537-45.

125. Martinez FJ, de Andrade JA, Anstrom KJ, King TE, Jr., Raghu G. Randomized trial of acetylcysteine in idiopathic pulmonary fibrosis. The New England journal of medicine. 2014;370(22):2093-101.

126. Spagnolo P, Maher TM. Clinical trial research in focus: why do so many clinical trials fail in IPF? The Lancet Respiratory medicine. 2017;5(5):372-4. 127. Jones DP. Redefining oxidative stress. Antioxidants \& redox signaling. 2006;8(9-10):1865-79.

128. Sies H. Oxidative stress: a concept in redox biology and medicine. 
Redox biology. 2015;4:180-3.

129. Mirzahosseini A, Noszal B. Species-Specific Standard Redox Potential of Thiol-Disulfide Systems: A Key Parameter to Develop Agents against Oxidative Stress. Scientific reports. 2016;6:37596.

130. Poole LB. The basics of thiols and cysteines in redox biology and chemistry. Free radical biology \& medicine. 2015;80:148-57.

131. Go YM, Jones DP. Redox compartmentalization in eukaryotic cells. Biochimica et biophysica acta. 2008;1780(11):1273-90.

132. Bilan DS, Shokhina AG, Lukyanov SA, Belousov VV. [Main Cellular Redox Couples]. Bioorganicheskaia khimiia. 2015;41(4):385-402.

133. Jones DP, Go YM. Redox compartmentalization and cellular stress. Diabetes, obesity \& metabolism. 2010;12 Suppl 2:116-25.

134. Jones DP, Liang Y. Measuring the poise of thiol/disulfide couples in vivo. Free radical biology \& medicine. 2009;47(10):1329-38.

135. Zhang P, Wu J, Xiao F, Zhao D, Luan Y. Disulfide bond based polymeric drug carriers for cancer chemotherapy and relevant redox environments in mammals. Medicinal research reviews. 2018;38(5):1485-510.

136. Jones DP, Mody VC, Jr., Carlson JL, Lynn MJ, Sternberg P, Jr. Redox analysis of human plasma allows separation of pro-oxidant events of aging from decline in antioxidant defenses. Free radical biology \& medicine. 2002;33(9):1290-300.

137. Go YM, Jones DP. Redox theory of aging: implications for health and 
disease. Clinical science (London, England : 1979). 2017;131(14):1669-88.

138. Moriarty SE, Shah JH, Lynn M, Jiang S, Openo K, Jones DP, et al. Oxidation of glutathione and cysteine in human plasma associated with smoking. Free radical biology \& medicine. 2003;35(12):1582-8.

139. Jonas CR, Puckett AB, Jones DP, Griffith DP, Szeszycki EE, Bergman GF, et al. Plasma antioxidant status after high-dose chemotherapy: a randomized trial of parenteral nutrition in bone marrow transplantation patients. The American journal of clinical nutrition. 2000;72(1):181-9.

140. Kinscherf R, Cafaltzis K, Roder F, Hildebrandt W, Edler L, Deigner HP, et al. Cholesterol levels linked to abnormal plasma thiol concentrations and thiol/disulfide redox status in hyperlipidemic subjects. Free radical biology \& medicine. 2003;35(10):1286-92.

141. Park Y, Ziegler TR, Gletsu-Miller N, Liang Y, Yu T, Accardi CJ, et al. Postprandial cysteine/cystine redox potential in human plasma varies with meal content of sulfur amino acids. The Journal of nutrition. 2010;140(4):760-5.

142. Yeh MY, Burnham EL, Moss M, Brown LA. Chronic alcoholism alters systemic and pulmonary glutathione redox status. American journal of respiratory and critical care medicine. 2007;176(3):270-6.

143. Schafer FQ, Buettner GR. Redox environment of the cell as viewed through the redox state of the glutathione disulfide/glutathione couple. Free radical biology \& medicine. 2001;30(11):1191-212.

144. Go YM, Jones DP. Intracellular proatherogenic events and cell 
adhesion modulated by extracellular thiol/disulfide redox state. Circulation. 2005;111(22):2973-80.

145. Jonas CR, Gu LH, Nkabyo YS, Mannery YO, Avissar NE, Sax HC, et al. Glutamine and KGF each regulate extracellular thiol/disulfide redox and enhance proliferation in Caco-2 cells. American journal of physiology Regulatory, integrative and comparative physiology. 2003;285(6):R1421-9.

146. Jiang S, Moriarty-Craige SE, Orr M, Cai J, Sternberg P, Jr., Jones DP. Oxidant-induced apoptosis in human retinal pigment epithelial cells: dependence on extracellular redox state. Investigative ophthalmology \& visual science. 2005;46(3):1054-61.

147. Go YM, Jones DP. Cysteine/cystine redox signaling in cardiovascular disease. Free radical biology \& medicine. 2011;50(4):495-509.

148. Ashfaq S, Abramson JL, Jones DP, Rhodes SD, Weintraub WS, Hooper WC, et al. The relationship between plasma levels of oxidized and reduced thiols and early atherosclerosis in healthy adults. Journal of the American College of Cardiology. 2006;47(5):1005-11.

149. Samiec PS, Drews-Botsch C, Flagg EW, Kurtz JC, Sternberg P, Jr., Reed RL, et al. Glutathione in human plasma: decline in association with aging, age-related macular degeneration, and diabetes. Free radical biology \& medicine. 1998;24(5):699-704.

150. Watson WH, Ritzenthaler JD, Peyrani P, Wiemken TL, Furmanek S, Reyes Vega AM, et al. Plasma cysteine/cystine and glutathione/glutathione 
disulfide redox potentials in HIV and COPD patients. Free radical biology \& medicine. 2019;143:55-61.

151. Ghosh D, Brewer GJ. External cys/cySS redox state modification controls the intracellular redox state and neurodegeneration via Akt in aging and Alzheimer's disease mouse model neurons. Journal of Alzheimer's disease : JAD. 2014;42(1):313-24.

152. Dong Y, Sameni S, Digman MA, Brewer GJ. Reversibility of Age-related Oxidized Free NADH Redox States in Alzheimer's Disease Neurons by Imposed External Cys/CySS Redox Shifts. Scientific reports. 2019;9(1):11274. 153. Zhu JW, Yuan JF, Yang HM, Wang ST, Zhang CG, Sun LL, et al. Extracellular cysteine (Cys)/cystine (CySS) redox regulates metabotropic glutamate receptor 5 activity. Biochimie. 2012;94(3):617-27 .

154. Chaiswing L, Zhong W, Liang Y, Jones DP, Oberley TD. Regulation of prostate cancer cell invasion by modulation of extra- and intracellular redox balance. Free radical biology \& medicine. 2012;52(2):452-61.

155. Nkabyo YS, Go YM, Ziegler TR, Jones DP. Extracellular cysteine/cystine redox regulates the p44/p42 MAPK pathway by metalloproteinase-dependent epidermal growth factor receptor signaling. American journal of physiology Gastrointestinal and liver physiology. 2005;289(1):G70-8.

156. Ramirez A, Ramadan B, Ritzenthaler JD, Rivera HN, Jones DP, Roman J. Extracellular cysteine/cystine redox potential controls lung fibroblast 
proliferation and matrix expression through upregulation of transforming growth factor-beta. American journal of physiology Lung cellular and molecular physiology. 2007;293(4):L972-81.

157. Watson WH, Burke TJ, Zelko IN, Torres-Gonzalez E, Ritzenthaler JD, Roman J. Differential Regulation of the Extracellular Cysteine/Cystine Redox State (EhCySS) by Lung Fibroblasts from Young and Old Mice. Oxidative medicine and cellular longevity. 2016;2016:1561305.

158. Huang Y, Dai Z, Barbacioru C, Sadee W. Cystine-glutamate transporter SLC7A11 in cancer chemosensitivity and chemoresistance. Cancer research. 2005;65(16):7446-54.

159. Mastroberardino L, Spindler B, Pfeiffer R, Skelly PJ, Loffing J, Shoemaker CB, et al. Amino-acid transport by heterodimers of $4 \mathrm{~F} 2 \mathrm{hc} / \mathrm{CD} 98$ and members of a permease family. Nature. 1998;395(6699):288-91.

160. Huang $\mathrm{Y}$, Sadee W. Membrane transporters and channels in chemoresistance and -sensitivity of tumor cells. Cancer letters. 2006;239(2):168-82.

161. Jones DP, Go YM, Anderson CL, Ziegler TR, Kinkade JM, Jr., Kirlin WG. Cysteine/cystine couple is a newly recognized node in the circuitry for biologic redox signaling and control. FASEB journal : official publication of the Federation of American Societies for Experimental Biology. 2004;18(11):12468.

162. Jones DP, Carlson JL, Mody VC, Cai J, Lynn MJ, Sternberg P. Redox 
state of glutathione in human plasma. Free radical biology \& medicine. 2000;28(4):625-35.

163. Go YM, Jones DP. The redox proteome. The Journal of biological chemistry. 2013;288(37):26512-20.

164. Watson $\mathrm{WH}$, Chen $\mathrm{Y}$, Jones DP. Redox state of glutathione and thioredoxin in differentiation and apoptosis. BioFactors (Oxford, England). 2003;17(1-4):307-14.

165. Jonas CR, Ziegler TR, Gu LH, Jones DP. Extracellular thiol/disulfide redox state affects proliferation rate in a human colon carcinoma (Caco2) cell line. Free radical biology \& medicine. 2002;33(11):1499-506.

166. lyer SS, Accardi CJ, Ziegler TR, Blanco RA, Ritzenthaler JD, Rojas M, et al. Cysteine redox potential determines pro-inflammatory IL-1beta levels. PloS one. 2009;4(3):e5017.

167. Liang LP, Patel M. Plasma cysteine/cystine redox couple disruption in animal models of temporal lobe epilepsy. Redox Biol. 2016;9:45-9.

168. Hall MN, Niedzwiecki M, Liu X, Harper KN, Alam S, Slavkovich V, et al. Chronic arsenic exposure and blood glutathione and glutathione disulfide concentrations in bangladeshi adults. Environ Health Perspect. 2013;121(9):1068-74.

169. Mannery YO, Ziegler TR, Park Y, Jones DP. Oxidation of plasma cysteine/cystine and GSH/GSSG redox potentials by acetaminophen and sulfur amino acid insufficiency in humans. J Pharmacol Exp Ther. 2010;333(3):939- 
47.

170. Rose S, Melnyk S, Trusty TA, Pavliv O, Seidel L, Li J, et al. Intracellular and extracellular redox status and free radical generation in primary immune cells from children with autism. Autism Res Treat. 2012;2012:986519.

171. Miller LT, Watson WH, Kirlin WG, Ziegler TR, Jones DP. Oxidation of the glutathione/glutathione disulfide redox state is induced by cysteine deficiency in human colon carcinoma HT29 cells. J Nutr. 2002;132(8):2303-6.

172. Sato $\mathrm{H}$, Tamba M, Ishii T, Bannai S. Cloning and expression of a plasma membrane cystine/glutamate exchange transporter composed of two distinct proteins. The Journal of biological chemistry. 1999;274(17):11455-8.

173. Sato H, Shiiya A, Kimata M, Maebara K, Tamba M, Sakakura Y, et al. Redox imbalance in cystine/glutamate transporter-deficient mice. The Journal of biological chemistry. 2005;280(45):37423-9.

174. Vene R, Delfino L, Castellani P, Balza E, Bertolotti M, Sitia R, et al. Redox remodeling allows and controls B-cell activation and differentiation. Antioxid Redox Signal. 2010;13(8):1145-55.

175. Lu SC. Glutathione synthesis. Biochimica et biophysica acta. 2013;1830(5):3143-53.

176. Roman J, Ritzenthaler JD, Gil-Acosta A, Rivera HN, Roser-Page S. Nicotine and fibronectin expression in lung fibroblasts: implications for tobaccorelated lung tissue remodeling. FASEB journal : official publication of the Federation of American Societies for Experimental Biology. 2004;18(12):1436- 
8.

177. Jones DP. Redox potential of GSH/GSSG couple: assay and biological significance. Methods in enzymology. 2002;348:93-112.

178. Jones DP, Carlson JL, Samiec PS, Sternberg P, Jr., Mody VC, Jr., Reed $\mathrm{RL}$, et al. Glutathione measurement in human plasma. Evaluation of sample collection, storage and derivatization conditions for analysis of dansyl derivatives by HPLC. Clinica chimica acta; international journal of clinical chemistry. 1998;275(2):175-84.

179. Nkabyo YS, Ziegler TR, Gu LH, Watson WH, Jones DP. Glutathione and thioredoxin redox during differentiation in human colon epithelial (Caco-2) cells. American journal of physiology Gastrointestinal and liver physiology. 2002;283(6):G1352-9.

180. Livak KJ, Schmittgen TD. Analysis of relative gene expression data using real-time quantitative PCR and the 2(-Delta Delta $\mathrm{C}(\mathrm{T})$ ) Method. Methods (San Diego, Calif). $2001 ; 25(4): 402-8$.

181. Mann GE. Nrf2-mediated redox signalling in vascular health and disease. Free Radic Biol Med. 2014;75 Suppl 1:S1.

182. Dinkova-Kostova AT, Fahey JW, Kostov RV, Kensler TW. KEAP1 and Done? Targeting the NRF2 Pathway with Sulforaphane. Trends Food Sci Technol. 2017;69(Pt B):257-69.

183. Bridges RJ, Natale NR, Patel SA. System xc(-) cystine/glutamate antiporter: an update on molecular pharmacology and roles within the CNS. 
British journal of pharmacology. 2012;165(1):20-34.

184. Bannai S, Ishii T. Formation of sulfhydryl groups in the culture medium by human diploid fibroblasts. J Cell Physiol. 1980;104(2):215-23.

185. Bannai S, Ishii T. Transport of cystine and cysteine and cell growth in cultured human diploid fibroblasts: effect of glutamate and homocysteate. Journal of cellular physiology. 1982;112(2):265-72.

186. Pader I, Sengupta R, Cebula M, Xu J, Lundberg JO, Holmgren A, et al. Thioredoxin-related protein of $14 \mathrm{kDa}$ is an efficient L-cystine reductase and Sdenitrosylase. Proceedings of the National Academy of Sciences of the United States of America. 2014;111(19):6964-9.

187. Anderson CL, lyer SS, Ziegler TR, Jones DP. Control of extracellular cysteine/cystine redox state by HT-29 cells is independent of cellular glutathione. Am J Physiol Regul Integr Comp Physiol. 2007;293(3):R1069-75. 188. Lu SC. Regulation of glutathione synthesis. Molecular aspects of medicine. 2009;30(1-2):42-59.

189. Burdo J, Dargusch R, Schubert D. Distribution of the cystine/glutamate antiporter system $\mathrm{xc}$ - in the brain, kidney, and duodenum. J Histochem Cytochem. 2006;54(5):549-57.

190. Banjac A, Perisic T, Sato H, Seiler A, Bannai S, Weiss N, et al. The cystine/cysteine cycle: a redox cycle regulating susceptibility versus resistance to cell death. Oncogene. 2008;27(11):1618-28.

191. Ishii T, Mann GE. Redox status in mammalian cells and stem cells 
during culture in vitro: critical roles of Nrf2 and cystine transporter activity in the maintenance of redox balance. Redox Biol. 2014;2:786-94.

192. Okuno S, Sato H, Kuriyama-Matsumura K, Tamba M, Wang H, Sohda $\mathrm{S}$, et al. Role of cystine transport in intracellular glutathione level and cisplatin resistance in human ovarian cancer cell lines. $\mathrm{Br} J$ Cancer. 2003;88(6):951-6.

193. Sleire L, Skeie BS, Netland IA, Forde HE, Dodoo E, Selheim F, et al. Drug repurposing: sulfasalazine sensitizes gliomas to gamma knife radiosurgery by blocking cystine uptake through system Xc-, leading to glutathione depletion. Oncogene. 2015;34(49):5951-9.

194. Balthasar C, Stangl H, Widhalm R, Granitzer S, Hengstschlager M, Gundacker C. Methylmercury Uptake into BeWo Cells Depends on LAT2-4F2hc, a System L Amino Acid Transporter. Int J Mol Sci. 2017;18(8).

195. Lewerenz J, Hewett SJ, Huang Y, Lambros M, Gout PW, Kalivas PW, et al. The cystine/glutamate antiporter system $\times(c)(-)$ in health and disease: from molecular mechanisms to novel therapeutic opportunities. Antioxidants \& redox signaling. 2013;18(5):522-55.

196. Kwak MK, Kensler TW. Targeting NRF2 signaling for cancer chemoprevention. Toxicol Appl Pharmacol. 2010;244(1):66-76.

197. Sestili P, Fimognari C. Cytotoxic and Antitumor Activity of Sulforaphane: The Role of Reactive Oxygen Species. Biomed Res Int. 2015;2015:402386. 198. Leone A, Diorio G, Sexton W, Schell M, Alexandrow M, Fahey JW, et al. Sulforaphane for the chemoprevention of bladder cancer: molecular 
mechanism targeted approach. Oncotarget. 2017;8(21):35412-24.

199. Veeranki OL, Bhattacharya A, Tang L, Marshall JR, Zhang Y. Cruciferous vegetables, isothiocyanates, and prevention of bladder cancer. Curr Pharmacol Rep. 2015;1(4):272-82.

200. Imhoff BR, Hansen JM. Extracellular redox status regulates Nrf2 activation through mitochondrial reactive oxygen species. Biochem $\mathrm{J}$. 2009;424(3):491-500.

201. Angelini G, Gardella S, Ardy M, Ciriolo MR, Filomeni G, Di Trapani G, et al. Antigen-presenting dendritic cells provide the reducing extracellular microenvironment required for T lymphocyte activation. Proc Natl Acad Sci U S A. 2002;99(3):1491-6.

202. Jones DP. Redox theory of aging. Redox biology. 2015;5:71-9.

203. Zheng Y, Ritzenthaler JD, Burke TJ, Otero J, Roman J, Watson WH. Age-dependent oxidation of extracellular cysteine/cystine redox state (Eh(Cys/CySS)) in mouse lung fibroblasts is mediated by a decline in Slc7a11 expression. Free radical biology \& medicine. 2018;118:13-22.

204. Truong TH, Carroll KS. Redox regulation of epidermal growth factor receptor signaling through cysteine oxidation. Biochemistry. 2012;51(50):995465.

205. Huang X, Begley M, Morgenstern KA, Gu Y, Rose P, Zhao H, et al. Crystal structure of an inactive Akt2 kinase domain. Structure (London, England : 1993). 2003;11(1):21-30. 
206. Wani R, Qian J, Yin L, Bechtold E, King SB, Poole LB, et al. Isoformspecific regulation of Akt by PDGF-induced reactive oxygen species. Proceedings of the National Academy of Sciences of the United States of America. 2011;108(26):10550-5.

207. Go YM, Orr M, Jones DP. Actin cytoskeleton redox proteome oxidation by cadmium. American journal of physiology Lung cellular and molecular physiology. 2013;305(11):L831-43.

208. Go YM, Chandler JD, Jones DP. The cysteine proteome. Free radical biology \& medicine. 2015;84:227-45.

209. Mermelekas G, Makridakis M, Koeck T, Vlahou A. Redox proteomics: from residue modifications to putative biomarker identification by gel- and LCMS-based approaches. Expert review of proteomics. 2013;10(6):537-49.

210. Chouchani ET, Kazak L, Jedrychowski MP, Lu GZ, Erickson BK, Szpyt $\mathrm{J}$, et al. Mitochondrial ROS regulate thermogenic energy expenditure and sulfenylation of UCP1. Nature. 2016;532(7597):112-6.

211. Shi X, Wei X, Koo I, Schmidt RH, Yin X, Kim SH, et al. Metabolomic analysis of the effects of chronic arsenic exposure in a mouse model of dietinduced Fatty liver disease. Journal of proteome research. 2014;13(2):547-54. 212. Shakir S, Vinh J, Chiappetta G. Quantitative analysis of the cysteine redoxome by iodoacetyl tandem mass tags. Analytical and bioanalytical chemistry. 2017;409(15):3821-30.

213. Kramer A, Green J, Pollard J, Jr., Tugendreich S. Causal analysis 
approaches in Ingenuity Pathway Analysis. Bioinformatics (Oxford, England). 2014;30(4):523-30.

214. Ashburner M, Ball CA, Blake JA, Botstein D, Butler H, Cherry JM, et al. Gene ontology: tool for the unification of biology. The Gene Ontology Consortium. Nature genetics. 2000;25(1):25-9.

215. Kanehisa M, Goto S. KEGG: kyoto encyclopedia of genes and genomes. Nucleic acids research. 2000;28(1):27-30.

216. Huang da W, Sherman BT, Lempicki RA. Systematic and integrative analysis of large gene lists using DAVID bioinformatics resources. Nature protocols. 2009;4(1):44-57.

217. von Mering C, Huynen M, Jaeggi D, Schmidt S, Bork P, Snel B. STRING: a database of predicted functional associations between proteins. Nucleic acids research. 2003;31(1):258-61.

218. Shannon P, Markiel A, Ozier O, Baliga NS, Wang JT, Ramage D, et al. Cytoscape: a software environment for integrated models of biomolecular interaction networks. Genome research. 2003;13(11):2498-504.

219. Roca-Cusachs P, del Rio A, Puklin-Faucher E, Gauthier NC, Biais N, Sheetz MP. Integrin-dependent force transmission to the extracellular matrix by alpha-actinin triggers adhesion maturation. Proceedings of the National Academy of Sciences of the United States of America. 2013;110(15):E1361-70. 220. Chinthalapudi K, Rangarajan ES, Izard T. The interaction of talin with the cell membrane is essential for integrin activation and focal adhesion 
formation. Proceedings of the National Academy of Sciences of the United States of America. 2018;115(41):10339-44.

221. Watson WH, Ritzenthaler JD, Roman J. Lung extracellular matrix and redox regulation. Redox biology. 2016;8:305-15.

222. Herrera J, Henke CA, Bitterman PB. Extracellular matrix as a driver of progressive fibrosis. The Journal of clinical investigation. 2018;128(1):45-53.

223. Poon HF, Castegna A, Farr SA, Thongboonkerd V, Lynn BC, Banks WA, et al. Quantitative proteomics analysis of specific protein expression and oxidative modification in aged senescence-accelerated-prone 8 mice brain. Neuroscience. 2004;126(4):915-26.

224. Perluigi M, Swomley AM, Butterfield DA. Redox proteomics and the dynamic molecular landscape of the aging brain. Ageing research reviews. 2014;13:75-89.

225. Shelton MD, Chock PB, Mieyal JJ. Glutaredoxin: role in reversible protein s-glutathionylation and regulation of redox signal transduction and protein translocation. Antioxidants \& redox signaling. 2005;7(3-4):348-66.

226. Murdoch CE, Bachschmid MM, Matsui R. Regulation of neovascularization by S-glutathionylation via the Wnt5a/sFlt-1 pathway. Biochemical Society transactions. 2014;42(6):1665-70.

227. Sandbo N, Dulin N. Actin cytoskeleton in myofibroblast differentiation: ultrastructure defining form and driving function. Translational research : the journal of laboratory and clinical medicine. 2011;158(4):181-96. 
228. Qin Z, Fisher GJ, Voorhees JJ, Quan T. Actin cytoskeleton assembly regulates collagen production via TGF-beta type II receptor in human skin fibroblasts. Journal of cellular and molecular medicine. 2018;22(9):4085-96.

229. Margittai E, Sitia R. Oxidative protein folding in the secretory pathway and redox signaling across compartments and cells. Traffic (Copenhagen, Denmark). 2011;12(1):1-8.

230. Bolter B, Soll J, Schwenkert S. Redox meets protein trafficking. Biochimica et biophysica acta. 2015;1847(9):949-56 .

231. Hwang JS, Hwang JS, Chang I, Kim S. Age-associated decrease in proteasome content and activities in human dermal fibroblasts: restoration of normal level of proteasome subunits reduces aging markers in fibroblasts from elderly persons. The journals of gerontology Series A, Biological sciences and medical sciences. 2007;62(5):490-9.

232. Saez I, Vilchez D. The Mechanistic Links Between Proteasome Activity, Aging and Age-related Diseases. Current genomics. 2014;15(1):38-51 .

233. Hohn TJ, Grune T. The proteasome and the degradation of oxidized proteins: part III-Redox regulation of the proteasomal system. Redox biology. 2014;2:388-94.

234. Hugo M, Korovila I, Kohler M, Garcia-Garcia C, Cabrera-Garcia JD, Marina A, et al. Early cysteine-dependent inactivation of $26 \mathrm{~S}$ proteasomes does not involve particle disassembly. Redox biology. 2018;16:123-8.

235. Reinheckel T, Sitte N, Ullrich O, Kuckelkorn U, Davies KJ, Grune T. 
Comparative resistance of the $20 \mathrm{~S}$ and $26 \mathrm{~S}$ proteasome to oxidative stress. The Biochemical journal. 1998;335 ( Pt 3):637-42.

236. Guo S, Wharton W, Moseley P, Shi H. Heat shock protein 70 regulates cellular redox status by modulating glutathione-related enzyme activities. Cell stress \& chaperones. 2007;12(3):245-54.

237. Sultana R, Boyd-Kimball D, Poon HF, Cai J, Pierce WM, Klein JB, et al. Redox proteomics identification of oxidized proteins in Alzheimer's disease hippocampus and cerebellum: an approach to understand pathological and biochemical alterations in AD. Neurobiology of aging. 2006;27(11):1564-76.

238. Butterfield DA, Perluigi M, Reed T, Muharib T, Hughes CP, Robinson RA, et al. Redox proteomics in selected neurodegenerative disorders: from its infancy to future applications. Antioxidants \& redox signaling. 2012;17(11):1610-55.

239. Silva GM, Netto LE, Simoes V, Santos LF, Gozzo FC, Demasi MA, et al. Redox control of $20 \mathrm{~S}$ proteasome gating. Antioxidants \& redox signaling. 2012;16(11):1183-94.

240. Tavernarakis N. Ageing and the regulation of protein synthesis: a balancing act? Trends in cell biology. 2008;18(5):228-35.

241. Anisimova AS, Alexandrov AI, Makarova NE, Gladyshev VN, Dmitriev SE. Protein synthesis and quality control in aging. Aging. 2018;10(12):4269-88. 242. Hashem Y, des Georges A, Dhote V, Langlois R, Liao HY, Grassucci RA, et al. Structure of the mammalian ribosomal $43 S$ preinitiation complex 
bound to the scanning factor DHX29. Cell. 2013;153(5):1108-19.

243. Jackson RJ, Hellen CU, Pestova TV. The mechanism of eukaryotic translation initiation and principles of its regulation. Nature reviews Molecular cell biology. 2010;11(2):113-27.

244. Richter JD, Sonenberg N. Regulation of cap-dependent translation by elF4E inhibitory proteins. Nature. 2005;433(7025):477-80.

245. Pyronnet S, Imataka H, Gingras AC, Fukunaga R, Hunter T, Sonenberg N. Human eukaryotic translation initiation factor $4 \mathrm{G}$ (elF4G) recruits mnk1 to phosphorylate elF4E. The EMBO journal. 1999;18(1):270-9.

246. Topf U, Suppanz I, Samluk L, Wrobel L, Boser A, Sakowska P, et al. Quantitative proteomics identifies redox switches for global translation modulation by mitochondrially produced reactive oxygen species. Nature communications. 2018;9(1):324.

247. Lasry A, Ben-Neriah Y. Senescence-associated inflammatory responses: aging and cancer perspectives. Trends in immunology. 2015;36(4):217-28.

248. Lin $\mathrm{CH}$, Lin PP, Lin CY, Lin CH, Huang CH, Huang YJ, et al. Decreased mRNA expression for the two subunits of system xc(-), SLC3A2 and SLC7A11, in WBC in patients with schizophrenia: Evidence in support of the hypoglutamatergic hypothesis of schizophrenia. Journal of psychiatric research. 2016;72:58-63.

249. Ji X, Qian J, Rahman SMJ, Siska PJ, Zou Y, Harris BK, et al. xCT 
(SLC7A11)-mediated metabolic reprogramming promotes non-small cell lung cancer progression. Oncogene. 2018;37(36):5007-19.

250. Tsuchihashi K, Okazaki S, Ohmura M, Ishikawa M, Sampetrean O, Onishi N, et al. The EGF Receptor Promotes the Malignant Potential of Glioma by Regulating Amino Acid Transport System xc(-). Cancer research. 2016;76(10):2954-63.

251. Isobe S, Kataoka M, Endo J, Moriyama H, Okazaki S, Tsuchihashi K, et al. Endothelial-Mesenchymal Transition Drives Expression of CD44 Variant and $\mathrm{xCT}$ in Pulmonary Hypertension. American journal of respiratory cell and molecular biology. 2019.

252. Hansen JM, Watson WH, Jones DP. Compartmentation of Nrf-2 redox control: regulation of cytoplasmic activation by glutathione and DNA binding by thioredoxin-1. Toxicological sciences : an official journal of the Society of Toxicology. 2004;82(1):308-17.

253. Watson $\mathrm{WH}$, Jones DP. Oxidation of nuclear thioredoxin during oxidative stress. FEBS letters. 2003;543(1-3):144-7.

254. Halvey PJ, Watson WH, Hansen JM, Go YM, Samali A, Jones DP. Compartmental oxidation of thiol-disulphide redox couples during epidermal growth factor signalling. The Biochemical journal. 2005;386(Pt 2):215-9.

255. Heilman JM, Burke TJ, McClain CJ, Watson WH. Transactivation of gene expression by NF-kappaB is dependent on thioredoxin reductase activity. Free radical biology \& medicine. 2011;51(8):1533-42. 
256. Barratt SL, Creamer A, Hayton C, Chaudhuri N. Idiopathic Pulmonary Fibrosis (IPF): An Overview. Journal of clinical medicine. 2018;7(8).

257. Sgalla G, lovene B, Calvello M, Ori M, Varone F, Richeldi L. Idiopathic pulmonary fibrosis: pathogenesis and management. Respiratory research. 2018;19(1):32.

258. Fernandez Perez ER, Daniels CE, Schroeder DR, St Sauver J, Hartman TE, Bartholmai BJ, et al. Incidence, prevalence, and clinical course of idiopathic pulmonary fibrosis: a population-based study. Chest. 2010;137(1):129-37.

259. Ley B, Collard HR, King TE, Jr. Clinical course and prediction of survival in idiopathic pulmonary fibrosis. American journal of respiratory and critical care medicine. 2011;183(4):431-40.

260. Yanai H, Shteinberg A, Porat Z, Budovsky A, Braiman A, Ziesche R, et al. Cellular senescence-like features of lung fibroblasts derived from idiopathic pulmonary fibrosis patients. Aging. 2015;7(9):664-72 .

261. Schafer MJ, White TA, lijima K, Haak AJ, Ligresti G, Atkinson EJ, et al. Cellular senescence mediates fibrotic pulmonary disease. Nature communications. 2017;8:14532.

262. Pardo A, Selman M, Ramirez R, Ramos C, Montano M, Stricklin G, et al. Production of collagenase and tissue inhibitor of metalloproteinases by fibroblasts derived from normal and fibrotic human lungs. Chest. 1992;102(4):1085-9. 
263. Verrey F, Closs El, Wagner CA, Palacin M, Endou H, Kanai Y. CATs and HATs: the SLC7 family of amino acid transporters. Pflugers Archiv : European journal of physiology. 2004;447(5):532-42.

264. Habib E, Linher-Melville K, Lin HX, Singh G. Expression of xCT and activity of system xc(-) are regulated by NRF2 in human breast cancer cells in response to oxidative stress. Redox biology. 2015;5:33-42.

265. Chen D, Fan Z, Rauh M, Buchfelder M, Eyupoglu IY, Savaskan N. ATF4 promotes angiogenesis and neuronal cell death and confers ferroptosis in a xCT-dependent manner. Oncogene. 2017;36(40):5593-608.

266. Lim JKM, Delaidelli A, Minaker SW, Zhang HF, Colovic M, Yang H, et al. Cystine/glutamate antiporter xCT (SLC7A11) facilitates oncogenic RAS transformation by preserving intracellular redox balance. Proceedings of the National Academy of Sciences of the United States of America. 2019;116(19):9433-42.

267. Harvey CJ, Thimmulappa RK, Singh A, Blake DJ, Ling G, Wakabayashi $\mathrm{N}$, et al. Nrf2-regulated glutathione recycling independent of biosynthesis is critical for cell survival during oxidative stress. Free radical biology \& medicine. 2009;46(4):443-53.

268. Bell KF, Fowler JH, Al-Mubarak B, Horsburgh K, Hardingham GE. Activation of Nrf2-regulated glutathione pathway genes by ischemic preconditioning. Oxidative medicine and cellular longevity. 2011;2011:689524. 269. Yamamoto M, Kensler TW, Motohashi H. The KEAP1-NRF2 System: a 
Thiol-Based Sensor-Effector Apparatus for Maintaining Redox Homeostasis. Physiological reviews. 2018;98(3):1169-203.

270. Suzuki T, Yamamoto M. Stress-sensing mechanisms and the physiological roles of the Keap1-Nrf2 system during cellular stress. The Journal of biological chemistry. 2017;292(41):16817-24.

271. Lau A, Whitman SA, Jaramillo MC, Zhang DD. Arsenic-mediated activation of the Nrf2-Keap1 antioxidant pathway. Journal of biochemical and molecular toxicology. 2013;27(2):99-105.

272. Sato H, Nomura S, Maebara K, Sato K, Tamba M, Bannai S. Transcriptional control of cystine/glutamate transporter gene by amino acid deprivation. Biochemical and biophysical research communications. 2004;325(1):109-16.

273. Kilberg MS, Shan J, Su N. ATF4-dependent transcription mediates signaling of amino acid limitation. Trends in endocrinology and metabolism: TEM. 2009;20(9):436-43.

274. Zhang P, McGrath BC, Reinert J, Olsen DS, Lei L, Gill S, et al. The GCN2 elF2alpha kinase is required for adaptation to amino acid deprivation in mice. Molecular and cellular biology. 2002;22(19):6681-8.

275. Lewerenz J, Maher P. Basal levels of elF2alpha phosphorylation determine cellular antioxidant status by regulating ATF4 and xCT expression. The Journal of biological chemistry. 2009;284(2):1106-15.

276. Jiang L, Kon N, Li T, Wang SJ, Su T, Hibshoosh H, et al. Ferroptosis as 
a p53-mediated activity during tumour suppression. Nature. 2015;520(7545):57-62.

277. Wang L, Liu Y, Du T, Yang H, Lei L, Guo M, et al. ATF3 promotes erastin-induced ferroptosis by suppressing system $\mathrm{Xc}()$. Cell death and differentiation. 2020;27(2):662-75.

278. Chen Y, Hu S, Mu L, Zhao B, Wang M, Yang N, et al. Slc7a11 Modulated by POU2F1 is Involved in Pigmentation in Rabbit. International journal of molecular sciences. 2019;20(10).

279. Nishizawa H, Matsumoto M, Shindo T, Saigusa D, Kato H, Suzuki K, et al. Ferroptosis is controlled by the coordinated transcriptional regulation of glutathione and labile iron metabolism by the transcription factor $\mathrm{BACH} 1$. The Journal of biological chemistry. 2020;295(1):69-82.

280. Vousden $\mathrm{KH}$, Lane DP. p53 in health and disease. Nature reviews Molecular cell biology. 2007;8(4):275-83.

281. Guo W, Zhao Y, Zhang Z, Tan N, Zhao F, Ge C, et al. Disruption of xCT inhibits cell growth via the ROS/autophagy pathway in hepatocellular carcinoma. Cancer letters. 2011;312(1):55-61.

282. Xie Y, Hou W, Song X, Yu Y, Huang J, Sun X, et al. Ferroptosis: process and function. Cell death and differentiation. 2016;23(3):369-79.

283. Hai T, Wolfgang CD, Marsee DK, Allen AE, Sivaprasad U. ATF3 and stress responses. Gene expression. 1999;7(4-6):321-35.

284. Okamoto A, Iwamoto Y, Maru Y. Oxidative stress-responsive 
transcription factor ATF3 potentially mediates diabetic angiopathy. Molecular and cellular biology. 2006;26(3):1087-97.

285. Dhakshinamoorthy S, Jain AK, Bloom DA, Jaiswal AK. Bach1 competes with Nrf2 leading to negative regulation of the antioxidant response element (ARE)-mediated $\mathrm{NAD}(\mathrm{P}) \mathrm{H}$ :quinone oxidoreductase 1 gene expression and induction in response to antioxidants. The Journal of biological chemistry. 2005;280(17):16891-900.

286. Warnatz HJ, Schmidt D, Manke T, Piccini I, Sultan M, Borodina T, et al. The BTB and CNC homology 1 (BACH1) target genes are involved in the oxidative stress response and in control of the cell cycle. The Journal of biological chemistry. 2011;286(26):23521-32 .

287. Zhang $\mathrm{Y}$, Koppula $\mathrm{P}$, Gan B. Regulation of $\mathrm{H} 2 \mathrm{~A}$ ubiquitination and SLC7A11 expression by BAP1 and PRC1. Cell cycle (Georgetown, Tex). 2019;18(8):773-83.

288. Zhang Y, Shi J, Liu X, Feng L, Gong Z, Koppula P, et al. BAP1 links metabolic regulation of ferroptosis to tumour suppression. Nature cell biology. 2018;20(10):1181-92.

289. Wang Y, Yang L, Zhang X, Cui W, Liu Y, Sun QR, et al. Epigenetic regulation of ferroptosis by $\mathrm{H} 2 \mathrm{~B}$ monoubiquitination and p53. EMBO reports. 2019;20(7):e47563.

290. Alver BH, Kim KH, Lu P, Wang X, Manchester HE, Wang W, et al. The SWI/SNF chromatin remodelling complex is required for maintenance of 
lineage specific enhancers. Nature communications. 2017;8:14648.

291. Wu JN, Roberts CW. ARID1A mutations in cancer: another epigenetic tumor suppressor? Cancer discovery. 2013;3(1):35-43.

292. Ogiwara H, Takahashi K, Sasaki M, Kuroda T, Yoshida H, Watanabe R, et al. Targeting the Vulnerability of Glutathione Metabolism in ARID1A-Deficient Cancers. Cancer cell. 2019;35(2):177-90.e8.

293. Wach S, Taubert H, Weigelt K, Hase N, Kohn M, Misiak D, et al. RNA Sequencing of Collecting Duct Renal Cell Carcinoma Suggests an Interaction between miRNA and Target Genes and a Predominance of Deregulated Solute Carrier Genes. Cancers. 2019;12(1).

294. Huang J, Jiang W, Tong X, Zhang L, Zhang Y, Fan H. Identification of gene and microRNA changes in response to smoking in human airway epithelium by bioinformatics analyses. Medicine. 2019;98(38):e17267.

295. Yu H, Han Z, Xu Z, An C, Xu L, Xin H. RNA sequencing uncovers the key long non-coding RNAs and potential molecular mechanism contributing to XAV939-mediated inhibition of non-small cell lung cancer. Oncology letters. 2019;17(6):4994-5004 .

296. Yue C, Ren Y, Ge H, Liang C, Xu Y, Li G, et al. Comprehensive analysis of potential prognostic genes for the construction of a competing endogenous RNA regulatory network in hepatocellular carcinoma. OncoTargets and therapy. 2019;12:561-76.

297. Chen P, Gu YY, Ma FC, He RQ, Li ZY, Zhai GQ, et al. Expression levels 
and cotargets of miRNA1263p and miRNA1265p in lung adenocarcinoma tissues: Alphan exploration with RTqPCR, microarray and bioinformatic analyses. Oncology reports. 2019;41(2):939-53.

298. Wei B, Kong W, Mou X, Wang S. Comprehensive analysis of tumor immune infiltration associated with endogenous competitive RNA networks in lung adenocarcinoma. Pathology, research and practice. 2019;215(1):159-70. 299. Wu Y, Sun X, Song B, Qiu X, Zhao J. MiR-375/SLC7A11 axis regulates oral squamous cell carcinoma proliferation and invasion. Cancer medicine. 2017;6(7):1686-97.

300. Drayton RM, Dudziec E, Peter S, Bertz S, Hartmann A, Bryant HE, et al. Reduced expression of miRNA-27a modulates cisplatin resistance in bladder cancer by targeting the cystine/glutamate exchanger SLC7A11. Clinical cancer research : an official journal of the American Association for Cancer Research. 2014;20(7):1990-2000.

301. Liu XX, Li XJ, Zhang B, Liang YJ, Zhou CX, Cao DX, et al. MicroRNA$26 \mathrm{~b}$ is underexpressed in human breast cancer and induces cell apoptosis by targeting SLC7A11. FEBS letters. 2011;585(9):1363-7.

302. Ponta H, Sherman L, Herrlich PA. CD44: from adhesion molecules to signalling regulators. Nature reviews Molecular cell biology. 2003;4(1):33-45.

303. Zoller M. CD44: can a cancer-initiating cell profit from an abundantly expressed molecule? Nature reviews Cancer. 2011;11(4):254-67.

304. Ishimoto T, Nagano O, Yae T, Tamada M, Motohara T, Oshima H, et al. 
CD44 variant regulates redox status in cancer cells by stabilizing the $\mathrm{xCT}$ subunit of system xc(-) and thereby promotes tumor growth. Cancer cell. 2011;19(3):387-400.

305. Horibe S, Kawauchi S, Tanahashi T, Sasaki N, Mizuno S, Rikitake Y. CD44v-dependent upregulation of $\mathrm{XCT}$ is involved in the acquisition of cisplatinresistance in human lung cancer A549cells. Biochemical and biophysical research communications. 2018;507(1-4):426-32.

306. Miyoshi S, Tsugawa H, Matsuzaki J, Hirata K, Mori H, Saya H, et al. Inhibiting xCT Improves 5-Fluorouracil Resistance of Gastric Cancer Induced by CD44 Variant 9 Expression. Anticancer research. 2018;38(11):6163-70.

307. Song Y, Park IS, Kim J, Seo HR. Actinomycin D inhibits the expression of the cystine/glutamate transporter XCT via attenuation of CD133 synthesis in CD133(+) HCC. Chemico-biological interactions. 2019;309:108713.

308. Shin CS, Mishra P, Watrous JD, Carelli V, D'Aurelio M, Jain M, et al. The glutamate/cystine xCT antiporter antagonizes glutamine metabolism and reduces nutrient flexibility. Nature communications. 2017;8:15074.

309. Song X, Zhu S, Chen P, Hou W, Wen Q, Liu J, et al. AMPK-Mediated BECN1 Phosphorylation Promotes Ferroptosis by Directly Blocking System Xc(-) Activity. Current biology : CB. 2018;28(15):2388-99.e5.

310. Liu R, Li X, Zhao G. Beclin1-mediated ferroptosis activation is associated with isoflurane-induced toxicity in SH-SY5Y neuroblastoma cells. Acta biochimica et biophysica Sinica. 2019;51(11):1134-41. 
311. Kang R, Zeh HJ, Lotze MT, Tang D. The Beclin 1 network regulates autophagy and apoptosis. Cell death and differentiation. 2011;18(4):571-80.

312. Liu T, Jiang L, Tavana O, Gu W. The Deubiquitylase OTUB1 Mediates Ferroptosis via Stabilization of SLC7A11. Cancer research. 2019;79(8):191324.

313. Gu Y, Albuquerque CP, Braas D, Zhang W, Villa GR, Bi J, et al. mTORC2 Regulates Amino Acid Metabolism in Cancer by Phosphorylation of the Cystine-Glutamate Antiporter xCT. Molecular cell. 2017;67(1):128-38.e7.

314. Wilson LE, Xu Z, Harlid S, White AJ, Troester MA, Sandler DP, et al. Alcohol and DNA Methylation: An Epigenome-Wide Association Study in Blood and Normal Breast Tissue. American journal of epidemiology. 2019;188(6):1055-65.

315. Xu K, Montalvo-Ortiz JL, Zhang X, Southwick SM, Krystal JH, Pietrzak $\mathrm{RH}$, et al. Epigenome-Wide DNA Methylation Association Analysis Identified Novel Loci in Peripheral Cells for Alcohol Consumption Among European American Male Veterans. Alcoholism, clinical and experimental research. 2019;43(10):2111-21.

316. Sato H, Tamba M, Kuriyama-Matsumura K, Okuno S, Bannai S. Molecular cloning and expression of human $\mathrm{xCT}$, the light chain of amino acid transport system xc. Antioxidants \& redox signaling. 2000;2(4):665-71.

317. Sato M, Kusumi R, Hamashima S, Kobayashi S, Sasaki S, Komiyama $\mathrm{Y}$, et al. The ferroptosis inducer erastin irreversibly inhibits system xc- and 
synergizes with cisplatin to increase cisplatin's cytotoxicity in cancer cells. Scientific reports. 2018;8(1):968.

318. Bannai S. Exchange of cystine and glutamate across plasma membrane of human fibroblasts. The Journal of biological chemistry. 1986;261(5):2256-63.

319. Koppula P, Zhang Y, Zhuang L, Gan B. Amino acid transporter SLC7A11/xCT at the crossroads of regulating redox homeostasis and nutrient dependency of cancer. Cancer communications (London, England). 2018;38(1):12.

320. Nakamura E, Sato M, Yang H, Miyagawa F, Harasaki M, Tomita K, et al. 4F2 (CD98) heavy chain is associated covalently with an amino acid transporter and controls intracellular trafficking and membrane topology of 4F2 heterodimer. The Journal of biological chemistry. 1999;274(5):3009-16.

321. Cantor JM, Ginsberg MH. CD98 at the crossroads of adaptive immunity and cancer. Journal of cell science. 2012;125(Pt 6):1373-82.

322. Torrents D, Estevez R, Pineda M, Fernandez E, Lloberas J, Shi YB, et al. Identification and characterization of a membrane protein $(y+L$ amino acid transporter-1) that associates with $4 \mathrm{~F} 2 \mathrm{hc}$ to encode the amino acid transport activity $y+L$. A candidate gene for lysinuric protein intolerance. The Journal of biological chemistry. 1998;273(49):32437-45.

323. Kanai $\mathrm{Y}$, Segawa H, Miyamoto K, Uchino H, Takeda E, Endou H. Expression cloning and characterization of a transporter for large neutral amino 
acids activated by the heavy chain of $4 \mathrm{~F} 2$ antigen (CD98). The Journal of biological chemistry. 1998;273(37):23629-32.

324. Fotiadis D, Kanai Y, Palacin M. The SLC3 and SLC7 families of amino acid transporters. Molecular aspects of medicine. 2013;34(2-3):139-58.

325. Conrad M, Sato $\mathrm{H}$. The oxidative stress-inducible cystine/glutamate antiporter, system $\times$ (c) (-) : cystine supplier and beyond. Amino acids. 2012;42(1):231-46.

326. Vitvitsky V, Thomas M, Ghorpade A, Gendelman HE, Banerjee R. A functional transsulfuration pathway in the brain links to glutathione homeostasis. The Journal of biological chemistry. 2006;281(47):35785-93.

327. Kandasamy P, Gyimesi G, Kanai Y, Hediger MA. Amino acid transporters revisited: New views in health and disease. Trends in biochemical sciences. 2018;43(10):752-89.

328. Wu G, Fang YZ, Yang S, Lupton JR, Turner ND. Glutathione metabolism and its implications for health. The Journal of nutrition. 2004;134(3):489-92.

329. Hirrlinger J, Dringen R. Multidrug resistance protein 1-mediated export of glutathione and glutathione disulfide from brain astrocytes. Methods in enzymology. 2005;400:395-409.

330. Rappa G, Lorico A, Flavell RA, Sartorelli AC. Evidence that the multidrug resistance protein (MRP) functions as a co-transporter of glutathione and natural product toxins. Cancer research. 1997;57(23):5232-7. 
331. Mueller CF, Widder JD, McNally JS, McCann L, Jones DP, Harrison DG. The role of the multidrug resistance protein- 1 in modulation of endothelial cell oxidative stress. Circulation research. 2005;97(7):637-44.

332. Yu X, Long YC. Crosstalk between cystine and glutathione is critical for the regulation of amino acid signaling pathways and ferroptosis. Scientific reports. 2016;6:30033.

333. Zhu J, Li S, Marshall ZM, Whorton AR. A cystine-cysteine shuttle mediated by $\mathrm{xCT}$ facilitates cellular responses to S-nitrosoalbumin. American journal of physiology Cell physiology. 2008;294(4):C1012-20.

334. Fomenko DE, Marino SM, Gladyshev VN. Functional diversity of cysteine residues in proteins and unique features of catalytic redox-active cysteines in thiol oxidoreductases. Molecules and cells. 2008;26(3):228-35.

335. Jones DP, Sies H. The Redox Code. Antioxidants \& redox signaling. 2015;23(9):734-46.

336. Yuan K, Liu Y, Chen HN, Zhang L, Lan J, Gao W, et al. Thiol-based redox proteomics in cancer research. Proteomics. 2015;15(2-3):287-99.

337. Pace NJ, Weerapana E. Zinc-binding cysteines: diverse functions and structural motifs. Biomolecules. 2014;4(2):419-34.

338. Hu K, Li K, Lv J, Feng J, Chen J, Wu H, et al. Suppression of the SLC7A11/glutathione axis causes synthetic lethality in KRAS-mutant lung adenocarcinoma. The Journal of clinical investigation. 2019.

339. Daher B, Parks SK, Durivault J, Cormerais Y, Baidarjad H, Tambutte E, 
et al. Genetic Ablation of the Cystine Transporter xCT in PDAC Cells Inhibits mTORC1, Growth, Survival, and Tumor Formation via Nutrient and Oxidative Stresses. Cancer research. 2019;79(15):3877-90.

340. Clemons NJ, Liu DS, Duong CP, Phillips WA. Inhibiting system xC(-) and glutathione biosynthesis - a potential Achilles' heel in mutant-p53 cancers. Molecular \& cellular oncology. 2017;4(5):e1344757.

341. Pekovic-Vaughan V, Gibbs J, Yoshitane H, Yang N, Pathiranage D, Guo $\mathrm{B}$, et al. The circadian clock regulates rhythmic activation of the NRF2/glutathione-mediated antioxidant defense pathway to modulate pulmonary fibrosis. Genes \& development. 2014;28(6):548-60. 


\section{LIST OF ABBREVIATIONS}

\begin{tabular}{ll} 
a-SMA & Alpha-smooth muscle actin \\
AARE & Amino acid response element \\
ACTA2 & Actin $\alpha$-2, smooth muscle \\
AD & Alzheimer disease \\
AEC & Alveolar epithelial cell \\
Akt2 & Serine/threonine kinase 2 \\
AMPK & AMP-activated protein kinase \\
ARE & Anti-oxidant response element \\
ARID1A & AT-rich interaction domain 1A \\
ATF4 & Activating transcription factor 4 \\
BACH1 & BTB and CNC homology 1 \\
BAL & Bronchoalveolar lavage \\
BAP1 & BRCA1-associated protein 1 \\
BECN1 & Beclin 1 \\
BP & Biological process \\
BSO & Buthionine sulfoximine \\
CAT & Catalase \\
CC & Cellular component \\
CD44 & Cluster of differentiation 44 \\
\hline
\end{tabular}




\begin{tabular}{ll} 
CD44v & Variant isoform of CD44 \\
CO2 & Carbon dioxide \\
COL1A1 & Collagen type I \\
COPD & Chronic obstructive pulmonary disease \\
CREB & cAMP response element binding protein \\
CRMP-2 & Collapsin response mediator protein-2 \\
CUL3 & Cullin 3 \\
Cys & Cysteine \\
CySS & Cystine \\
DAVID & Database for Annotation, Visualization and Integrated \\
ELF & Discovery \\
EMT & Dulbecco's Modified Essential Medium \\
DMEM & Exithelial-to-mesenchymal transition \\
EBC & Exhaled breath condensate \\
ECM & Extracellular matrix \\
EDTA & Ethylenediaminetetraacetic acid \\
EGFR & Epidermal growth factor receptor \\
EIF4E & Eukaryotic translation initiation factor $4 \mathrm{E}$ \\
ELF & Eukaryotic translation initiation factor $2 \alpha$ \\
\hline
\end{tabular}




$\begin{array}{ll}\text { EPO } & \text { Erythropoietin } \\ \text { ERK } & \text { Extracellular signal-regulated kinase } \\ \text { ETS-1 } & \text { ETS proto-oncogene 1, transcription factor } \\ \text { FBS } & \text { Fetal bovine serum } \\ \text { FDR } & \text { False discovery rate } \\ \text { FGF2 } & \text { Fibroblasts growth factor 2 } \\ \text { FLNB } & \text { Filamin B } \\ \text { GCL } & \text { Glutamate-cysteine ligase } \\ \text { GCLC } & \text { Catalytic subunit of glutamate-cysteine ligase } \\ \text { GCLM } & \text { Modifier subunit of glutamate-cysteine ligase } \\ \text { GCN2 } & \text { General control non-derepressible 2 } \\ \text { GGT } & \text { Gamma-glutamyl transferase } \\ \text { GO } & \text { Gene Ontology } \\ \text { GPX } & \text { Glutathione peroxidase } \\ \text { GRX } & \text { Glutaredoxin } \\ \text { GSH } & \text { Glutathione } \\ \text { GSS } & \text { Glutathione synthetase } \\ \text { GSSG } & \text { Glutathione disulfide } \\ \text { GST } & \text { Glutathione S-transferase } \\ \text { H2O } & \text { Water } \\ \text { H2Odrogen peroxide }\end{array}$




\begin{tabular}{|c|c|}
\hline HPLC & High-performance liquid chromatography \\
\hline HRCT & High-resolution computed tomography \\
\hline HSP70 & Heat shock protein 70 \\
\hline ICAT & Isotope-coded affinity tag \\
\hline IL-1 $\beta$ & Interleukin-1 $\beta$ \\
\hline ILK & Integrin-linked kinase \\
\hline iNOS & Inducible nitric oxide synthase \\
\hline lodoTMT & lodoacetyl tandem mass tag \\
\hline IPA & Ingenuity Pathway Analysis \\
\hline IPF & Idiopathic pulmonary fibrosis \\
\hline KEAP1 & Kelch like $\mathrm{ECH}$ associated protein 1 \\
\hline KEGG & Kyoto Encyclopedia of Genes and Genomes \\
\hline LAP & Latency-associated protein \\
\hline LC-MS/MS & Liquid chromatography-tandem mass spectrometry \\
\hline LPS & Lipopolysaccharide \\
\hline LTBP & Latent TGF- $\beta$ binding protein \\
\hline MAPK & Mitogen-activated protein kinase \\
\hline MDA & Malondialdehyde \\
\hline MF & Molecular function \\
\hline mGlu5 & Metabotropic glutamate receptor 5 \\
\hline miRNA & MicroRNA \\
\hline MMP & Matrix metallopeptidase \\
\hline
\end{tabular}




$\begin{array}{ll}\text { MRP } & \text { Multi-drug resistance protein } \\ \text { mTORC1 } & \text { Mammalian target of rapamycin complex 1 } \\ \text { NAC } & \text { N-acetylcysteine } \\ \text { NADH } & \text { Reduced nicotinamide adenine dinucleotide } \\ \text { NADPH } & \text { Reduced nicotinamide adenine dinucleotide phosphate } \\ \text { NF-KB } & \text { Nuclear factor-kB } \\ \text { NOX } & \text { NADPH oxidase } \\ \text { NRF2 } & \text { Nuclear factor, erythroid 2 like 2 } \\ \text { O2 } & \text { Oxygen } \\ \text { O2- } & \text { Superoxide anion } \\ \text { OTUB1 } & \text { OTU deubiquitinase, ubiquitin aldehyde binding } 1 \\ \text { PBS } & \text { Phosphate-buffered saline } \\ \text { PCA } & \text { Perchloric acid } \\ \text { Pin 1 } & \text { Peptidyl-prolyl cis-trans isomerase 1 } \\ \text { POU2F1 } & \text { POU class 2 homeobox 1 } \\ \text { PPI } & \text { Protein-protein interaction } \\ \text { PPP2CA } & \text { Protein phosphatase 2 catalytic subunit alpha } \\ \text { RRX } & \text { Peroxiredoxin } \\ \text { RPCR } & \text { Quantitative PCR } \\ \text { RBX1 } & \text { Ring-box 1 } \\ \text { Redox } & \text { Reduction and oxidation } \\ \text { Alcohol }\end{array}$




\begin{tabular}{|c|c|}
\hline $\mathrm{ROOH}$ & Organic hydroperoxide \\
\hline ROS & Reactive oxygen species \\
\hline RPL9 & Ribosomal protein L9 \\
\hline RPS6 & Ribosomal protein S6 \\
\hline SAMP8 & Senescence-accelerated mouse-prone 8 \\
\hline SASP & Senescence-associated secretory phenotype \\
\hline SDS & Sodium dodecyl sulfate \\
\hline Slc7a11 & Solute carrier family 7 , member 11 \\
\hline SOD & Superoxide dismutase \\
\hline SRX & Sulfiredoxin \\
\hline System ASC & System alanine-serine-cysteine \\
\hline TBS & Tris-buffered saline \\
\hline TCA & Trichloroacetic acid \\
\hline TCEP & Tris(2-carboxyethyl)phosphine \\
\hline TEAC & Trolox equivalent anti-oxidant capacity \\
\hline TGF- $\beta$ & Transforming growth factor- $\beta$ \\
\hline TLN1 & Talin 1 \\
\hline TNFa & Tumor necrosis factor $\alpha$ \\
\hline TRX & Thioredoxin \\
\hline Txndc17 & Thioredoxin domain containing 17 \\
\hline Txnrd1 & Thioredoxin reductase 1 \\
\hline UCHL-1 & Ubiquitin carboxyl terminal hydrolase L-1 \\
\hline
\end{tabular}


UIP

Usual interstitial pneumonia

USP7 Ubiquitin-specific peptidase 7

3'-UTR 3'-untranslated region

4F2hc 4F2 heavy chain

8-OHdG 8-hydroxy-deoxyguanosine 


\title{
CURRICULUM VITAE
}

\author{
Yuxuan Zheng \\ University of Louisville School of Medicine \\ Department of Pharmacology and Toxicology \\ Louisville, KY 40202 \\ E-mail: y0zhen14@louisville.edu
}

EDUCATION

2009-2014 Bachelor of Medicine,

Jilin University,

Changchun, Jilin, China

2014-2016 Master of Clinical Medicine,

Jilin University,

Changchun, Jilin, China

2016-Present Ph.D. candidate in Pharmacology and Toxicology,

University of Louisville,

Louisville, KY, USA 
PROFESSIONAL MEMBERSHIPS AND ACTIVITIES

2018-Present Society of Toxicology, student member

2019-Present American Thoracic Society, trainee ATS member

HONORS AND AWARDS

2009-2010 First-class scholarship of Jilin University

Excellent student of Jilin University

2010-2011 Third-class scholarship of Jilin University

Excellent student of the Medical College, Jilin University

First price of National English Contest for College Students in 2011

Good volunteer of the Seventh Challenge Cup

2012-2013 First-class scholarship of Jilin University

Excellent student of Jilin University

Second price of National English Contest for College Students in 2012

2018 University of Louisville Graduate Student Council Travel Awards 2018 Spring Semester

2019 University of Louisville Graduate Student Council Travel Awards 2019 Spring Semester

2019 University of Louisville Graduate Student Council Travel Awards 2019 Summer Semester 

Awards 2020 Spring Semester

2020 Society of Toxicity (SOT) Graduate Student Travel Support Award

2020 American Thoracic Society (ATS) 2020 Student Scholars Program

\section{ABSTRACTS/POSTERS}

2017 \#GRD-53 Research!Louisville,

Sep University of Louisville, Louisville, KY.

Zheng, Y, Ritzenthaler, JD, Burke, TJ, Otero, J, Roman, J, Watson, WH.

"Regulation of Cysteine/Cystine Redox State Eh(Cys/CySS) and Glutathione Synthesis by Age-dependent Slc7a11 Expression of Mouse Lung Fibroblasts."

2017 \#F-23 Research!Louisville,

Sep University of Louisville, Louisville, KY.

Watson, WH, Zheng, Y, Ritzenthaler, JD, Burke, TJ, TorresGonzalez, E, Roman, J.

"Ethanol Induces Fibronectin Expression through Redox Regulation of the Alpha-4 Nicotinic Acetylcholine Receptor (a4 nAChR)." 
2018 p.\#2445 Society of Toxicology (SOT) 57th Annual Meeting,

Mar San Antonio, TX.

Zheng, Y, Ritzenthaler, JD, Burke, TJ, Otero, J, Roman, J, Watson, WH.

"Regulation of Cysteine/Cystine Redox State Eh(Cys/CySS) and Glutathione Synthesis by Age-dependent SIc7a11 Expression of Mouse Lung Fibroblasts."

2018 p.\#2450 Society of Toxicology (SOT) 57th Annual Meeting,

Mar San Antonio, TX.

Watson, WH, Zheng, Y, Ritzenthaler, JD, Burke, TJ, Torres-

Gonzalez, E, Roman, J.

"Ethanol Induces Fibronectin Expression through Redox Regulation of the Alpha-4 Nicotinic Acetylcholine Receptor (a4 nAChR)."

2018 p.A3855 American Thoracic Society (ATS) Annual Meeting, May San Diego, CA.

Ritzenthaler, JD, Watson, WH, Peyrani, P, Wiemken, T, Burke, TJ, Zheng, Y, Ramirez, JA, Roman, J.

"Redox States of Cysteine/Cystine and Glutathione/Glutathione Disulfide in Plasma and Bronchoalveolar Lavage Fluid in HIVInfected Patients with COPD."

2018 p.A6350 American Thoracic Society (ATS) Annual Meeting, 
May San Diego, CA.

Ritzenthaler, JD, Zheng, Y, Burke, TJ, Otero, J, Roman, J, Watson, WH.

"Alterations in the Expression of Slc7a11 in Murine Lung Fibroblasts Is Responsible for Aging-Dependent Oxidation of Cysteine/Cystine Redox State (Eh Cys/CySS)."

2018 p.A6351 American Thoracic Society (ATS) Annual Meeting, May San Diego, CA.

Ritzenthaler, JD, Watson, WH, Zheng, Y, Burke, TJ, TorresGonzalez, E, Arteel, G, Roman, J.

"Role of Alpha 4 Nicotinic Acetylcholine Receptors (a4 nAChR) in Regulation of Fibronectin Expression and Redox State in Lung Fibroblasts."

2018 p.A6373 American Thoracic Society (ATS) Annual Meeting, May San Diego, CA.

Suliman, SA, Ritzenthaler, JD, Peyrani, P, Wiemken, T, Burke, TJ, Zheng, Y, Ramirez, JA, Watson, WH, Roman, J.

"Redox States of Thiol Disulfide Couples and Markers of Extracellular Matrix Remodeling in Bronchoalveolar Lavage Fluid of HIV-Infected Subjects with COPD."

2018 \#GRD-40 Research!Louisville,

Oct University of Louisville, Louisville, KY. 
Zheng, Y, Cai, J, Merchant, ML, Burke, TJ, Ritzenthaler, JD, Roman, J, Watson, WH.

"Redox Proteomics Analysis Reveals Slc7a11 Restores Agedependent Change of Redox State of Proteins in Pathways of Protein Turnover and Cell Death"

2018 p.26 Ohio Valley Society of Toxicology (OVSOT) Annual Meeting, Nov University of Louisville, Louisville, KY.

Zheng, Y, Cai, J, Merchant, ML, Burke, TJ, Ritzenthaler, JD, Roman, J, Watson, WH.

"Redox Proteomics Analysis Reveals Slc7a11 Restores Agedependent Change of Redox State of Proteins in Pathways of Protein Turnover and Cell Death"

2019 p.\#2003 Society of Toxicology (SOT) 58th Annual Meeting,

Mar Baltimore, MD.

Zheng, Y, Cai, J, Merchant, ML, Burke, TJ, Ritzenthaler, JD, Roman, J, Watson, WH.

"Redox Proteomics Analysis Reveals Slc7a11 Restores Agedependent Change of Redox State of Proteins in Pathways of Protein Turnover and Cell Death"

2019 \#GRD-59 Research!Louisville,

Sep University of Louisville, Louisville, KY. 
Zheng, Y, Burke, TJ, Ritzenthaler, JD, Zelko, IN, Van Berkel, V, Nunley, DR, Halayko, AJ, Roman, J, Watson, WH.

"Lung Fibroblasts from Idiopathic Pulmonary Fibrosis Patients Produce an Oxidizing Extracellular Redox Potential"

2019 \#GRD-18 Research!Louisville,

Sep University of Louisville, Louisville, KY.

Bushau-Sprinkle, A, Watson, WH, Zheng, Y, Gagnon, K, Kitterman, K, Barati, M, Siskind, L, Brier, M, Lederer, E.

"Mechanistic insight for increased susceptibility to cisplatin nephrotoxicity with NHERF1 loss"

2019 \#F-17 Research!Louisville,

Sep University of Louisville, Louisville, KY.

Watson, WH, Greenwell, JC, Zheng, Y, Torres-Gonzalez, E, Ritzenthaler, JD, Roman, J.

"Impact of sex, age, and diet on the cysteine/cystine and glutathione/glutathione disulfide plasma redox couples in mice"

2019 Abs\#3237151 American Society of Nephrology Kidney Week,

Nov Washington D.C.

Bushau-Sprinkle, A, Watson, WH, Zheng, Y, Gagnon, K, Kitterman, K, Barati, M, Siskind, L, Brier, M, Lederer, E.

"Mechanistic insight for increased susceptibility to cisplatin nephrotoxicity with NHERF1 loss" 


\section{PLATFORM PRESENTATION}

2019 PLAT4 Ohio Valley Society of Toxicology (OVSOT) Annual Meeting, Oct Procter \& Gamble, Mason Business Center (MBC), Mason, $\mathrm{OH}$. "Lung Fibroblasts from Idiopathic Pulmonary Fibrosis Patients Produce an Oxidizing Extracellular Redox Potential”

\section{SEMINAR PRESENTATION}

2019 Jan Department of Pharmacology and Toxicology,

University of Louisville, Louisville, KY.

"Research Overview of Idiopathic Pulmonary Fibrosis"

\section{JOURNAL CLUB PRESENTATIONS}

2018 Apr Wang et al., J Clin Invest, 2018; 128(6):2297-2309.

"TRAP-seq identifies cystine/glutamate antiporter as a driver of recovery from liver injury"

2018 May Li et al., J Proteome Res, 2018; 17(1):129-140.

"Proteomic Analyses of Cysteine Redox in High-Fat-Fed and Fasted Mouse Livers: Implications for Liver Metabolic Homeostasis"

2018 Jun Ji et al., Oncogene, 2018; 37(36):5007-5019. 
"xCT (SLC7A11)-mediated metabolic reprogramming promotes non-small cell lung cancer progression."

2018 Aug Song et al., Curr Biol, 2018; 28(15):2388-2399.e5.

"AMPK-Mediated BECN1 Phosphorylation Promotes Ferroptosis by Directly Blocking System Xc- Activity."

2018 Oct Nichenametla et al., Ann N Y Acad Sci, 2018; 1418(1):80-94.

"Sulfur amino acid restriction-induced changes in redox-sensitive proteins are associated with slow protein synthesis rates."

$2018 \mathrm{Dec}$ Go et al., Am J Physiol Lung Cell Mol Physiol, 2013; 305(11):L831-43.

"Actin cytoskeleton redox proteome oxidation by cadmium."

2019 Apr Wen et al., Am J Pathol, 2014; 184(5):1299-308.

"Transgenic expression of the human MRP2 transporter reduces cisplatin accumulation and nephrotoxicity in Mrp2-null mice."

2019 May Isobe et al., Am J Respir Cell Mol Biol, 2019; 61(3):367-379.

"Endothelial-Mesenchymal Transition Drives Expression of CD44 Variant and xCT in Pulmonary Hypertension."

2019 Jun Tsuchihashi et al., Cancer Res, 2016; 76(10):2954-63.

"The EGF Receptor Promotes the Malignant Potential of Glioma by Regulating Amino Acid Transport System xc(-)."

2019 Sep Kyung et al., BMC Pharmacol Toxicol, 2018; 19(1):13. 
"Sulforaphane attenuates pulmonary fibrosis by inhibiting the epithelial-mesenchymal transition."

2019 Nov Choi et al., Cell Metab, 2019;30(5):877-889.e7.

"Glutamate Signaling in Hepatic Stellate Cells Drives Alcoholic Steatosis."

2019 Dec Elko et al., Free Radic Biol Med, 2019; 141:438-446.

"Age-dependent dysregulation of redox genes may contribute to fibrotic pulmonary disease susceptibility."

2019 Dec Veith et al., Antioxid Redox Signal, 2019; 31(14):1092-1115.

"Redox Imbalance in Idiopathic Pulmonary Fibrosis: A Role for Oxidant Cross-Talk Between NADPH Oxidase Enzymes and Mitochondria."

2020 Feb Coronavirus update - literature review

\section{PUBLICATIONS}

1. Han Q, Cheng K, Zhong H, Gao Q, Chen X, Zheng Y, Shao P, Wang Y, Li Y, Wang J. Linear measurements of healthy adults' coronal section of hippocampus on brain magnetic resonance imaging. J Craniofac Surg. 2013 Jan;24(1):197-9. PMID: 23348285

2. Zheng Y, Liu L, Wang S, Li Z, Hou M, Li J, Yu XF, Zhang W, Hua S. Prevailing genotype distribution and characteristics of human respiratory syncytial virus in northeastern China. J Med Virol. 2017 Feb;89(2):222-233. PMID: 27448044 
3. Zheng Y, Ritzenthaler JD, Burke TJ, Otero J, Roman J, Watson WH. Agedependent oxidation of extracellular cysteine/cystine redox state (Eh(Cys/CySS)) in mouse lung fibroblasts is mediated by a decline in Slc7a11 expression. Free Radic Biol Med. 2018 Apr;118:13-22. PMID: 29458149

4. Watson WH, Ritzenthaler JD, Peyrani P, Wiemken TL, Furmanek S, Reyes Vega AM, Burke TJ, Zheng Y, Ramirez JA, Roman J. Plasma cysteine/cystine and glutathione/glutathione disulfide redox potentials in HIV and COPD patients. Free Radic Biol Med. 2019 Jul;143:55-61. PMID: 31369840

5. Zheng Y, Cai J, Merchant ML, Burke TJ, Ritzenthaler JD, Gaweda AE, Roman J, Watson WH. Redox states of protein cysteines in pathways of protein turnover and cytoskeleton dynamics are changed with aging and reversed by Slc7a11 restoration in mouse lung fibroblasts. (Under Major Revision, Oxidative Medicine and Cellular Longevity)

6. Watson $\mathrm{WH}^{\star}$, Greenwell, JC* ${ }^{\star}$ Zheng Y, Torres-González E, Ritzenthaler JD, Roman J. Impact of sex, age, and diet on the cysteine/cystine and glutathione/glutathione disulfide plasma redox couples in mice. (Re-submitted to The Journal of Nutritional Biochemistry after Major Revision)

7. Zheng Y, Hu J, Wang Y, Zhu Y, Li Y, Hao R, Qi Y. Prognostic and clinicopathological significance of circRNAs in lung cancer of the Chinese population: a systematic review and meta-analysis. (Under Review, Journal of Cancer) 